\title{
Periplasmic flagella of the spirochetes Borrelia burgdorferi and Brachyspira hyodysenteriae
}

Melanie S. Sal

West Virginia University

Follow this and additional works at: https://researchrepository.wvu.edu/etd

\section{Recommended Citation}

Sal, Melanie S., "Periplasmic flagella of the spirochetes Borrelia burgdorferi and Brachyspira hyodysenteriae" (2005). Graduate Theses, Dissertations, and Problem Reports. 2280.

https://researchrepository.wvu.edu/etd/2280

This Dissertation is protected by copyright and/or related rights. It has been brought to you by the The Research Repository @ WVU with permission from the rights-holder(s). You are free to use this Dissertation in any way that is permitted by the copyright and related rights legislation that applies to your use. For other uses you must obtain permission from the rights-holder(s) directly, unless additional rights are indicated by a Creative Commons license in the record and/ or on the work itself. This Dissertation has been accepted for inclusion in WVU Graduate Theses, Dissertations, and Problem Reports collection by an authorized administrator of The Research Repository @ WVU.

For more information, please contact researchrepository@mail.wvu.edu. 
Periplasmic Flagella of the Spirochetes Borrelia burgdorferi and Brachyspira hyodysenteriae

Melanie S. Sal

Dissertation submitted to the School of Medicine at West Virginia University

in partial fulfillment of the requirements for the degree of

Doctor of Philosophy

In

Microbiology and Immunology

Nyles W. Charon Ph.D. (Chair)

David Yelton Ph.D.

Daniel Flynn Ph.D.

Rosana Schafer Ph.D.

Daniel Panaccione Ph.D.

Department of Microbiology, Immunology and Cell Biology

Morgantown, West Virginia

2005

Keywords: Borrelia burgdorferi, Brachyspira hyodysenteriae, periplasmic flagella, Lyme disease, Swine dysentery

Copyright 2005 Melanie S. Sal 


\begin{abstract}
Periplasmic Flagella of the Spirochetes Borrelia burgdorferi and Brachyspira hyodysenteriae
\end{abstract}

Melanie S. Sal

Spirochetes are unique bacteria that cause a variety of diseases. Motility, provided by periplasmic flagella (PFs), is a virulence factor for spirochetes. This work focuses on PFs from Borrelia burgdorferi and Brachyspira hyodysenteriae. PFs consist of three main parts: basal body, hook, and filament. Monoclonal antibodies were developed to attempt identification of individual FlaB filament proteins in $B$. hyodysenteriae.

The PF filament of $B$. burgdorferi consists of a major flagellin, FlaB, and a minor flagellin, FlaA. FlaB constitutes $10-14 \%$, whereas FlaA contributes less than $0.5 \%$, of total cellular protein. Although these flagellins co-precipitate, the location of FlaA on a PF is unknown. FlaA accumulates at only $13 \%$ of wild type levels in the flaB null mutant, MC-1. Because mRNA levels are not negatively altered, FlaA regulation occurs at a posttranscriptional level. As FlaA is degraded over time, protein turnover may be a mechanism responsible for reduced $\mathrm{FlaA}$ accumulation in $\mathrm{MC}-1$.

The flagellar hook has structural and regulatory roles in many bacteria. The PF hook gene, flgE, is required for motility, wave-like cell morphology, and PF synthesis in $B$. burgdorferi. The flgE null mutant, SC-1, has markedly reduced levels of filament proteins FlaB and FlaA. Because flaB and flaA transcripts are not negatively altered, filament protein synthesis is regulated at a posttranscriptional level. As seen in MC-1, FlaA is degraded over time in SC-1. Conversely, FlaB is stable indicating that protein turnover is not a significant mechanism for maintaining reduced FlaB accumulation in SC-1.

In addition to a loss of FlgE in SC-1, a series of high molecular weight bands reactive to hook antisera was lost. This "ladder" was present in wild type strains B31 and N40, and also in MC-1 cells, all of which presumably assemble the PF hook. The ladder is absent in SC-1 and fliF null basal body mutants in which the PF hook cannot assemble. Furthermore, the ladder is present in purified PFs and in isolated hook-basal body complexes from B31 PFs. The ladder suggests that these bands are FlgE aggregates that are strongly associated, perhaps by cross-linking. The nature of FlgE protein association is currently unknown. 


\section{Dedication}

I dedicate this work to ...

My Father - for instilling in me a deep respect for nature, a passion for the incredible mysteries of life, the desire to explore my world, and an appreciation for patience and balance.

My Mother - for teaching me to congratulate the winners, to hold my head high, and to never back down.

My Sister - for challenging me, using big words, playing calculus, proving physics, and especially for never believing TV product advertising until we tried it ourselves (my very first experiments).

My Love - for embracing my free spirit, giving me faith, encouraging me to try again, and reminding me of what is really important in life. 


\section{Acknowledgements}

I wish to acknowledge those people who have impacted my life during the journey of graduate school.

I extend my sincerest thanks to my advisor, Dr. Nyles Charon, for his kind and generous guidance, for introducing me to the world of spirochetes, and for allowing me to be part of his laboratory. Nyles is not only a remarkable scientist, but also a truly amazing person.

I appreciate the members of my Committee for taking an active interest in my graduate career. I thank Dr. Dan Flynn for working so hard to help me overcome unusual circumstances during my initial admittance to graduate school. I thank Dr. Dan Panaccione for demonstrating excellence in teaching and genuine interest in student success. These are lessons I will take with me. I thank Dr. Rosana Schafer for being approachable, friendly, warm, and caring. She is absolutely a fantastic role model for finding balance as a woman in science. I thank Dr. David Yelton for his unfaltering dedication to the Charon lab. Dr. Yelton has been actively involved with the lab since I began and has acted as a second advisor to me. I thank him especially for rising above and going beyond the call of duty to hold the Charon lab together during difficult times. Your efforts are very much appreciated.

I recognize and thank professors and colleagues from West Virginia University and Japan who have contributed to my graduate career along the way. A special thanks is in order for Dr. Shin-Ichi Aizawa and the members of his laboratory. I appreciate Chi's interest in my education, both in the lab and out, and I am eternally grateful for his kind invitation and warm welcome to Japan. He is a scientist in the purest form and encourages others to pursue knowledge freely in his laboratory. I am fortunate and honored to know this incredible man.

There are no words to thank my friends enough. You have made my graduate experience unbelievable and unforgettable. To Sarah and Alicia: We built strong friendships and did this crazy thing together - start to finish. Thank you both for laughter, encouragement, and strength. To Richard: You have been a remarkable ally and friend. It was a pleasure and honor to be your labmate, pal, and random dance partner. To the gang: Cindy, Jeffers, Lisa, and Ed. What a wild ride, huh? You all have filled my heart with precious memories and moments that I will treasure forever. I cannot thank you enough for what you have given me.

Again, I thank my family: Tom, Janet, Natalie, Brian, and Bill. I would not be where I am today without your love and support. 
Table of Contents

Abstract__ ii

Dedication__ iii

Acknowledgements__ iv

Table of Contents _ $v$

Table of Figures _ viii

Chapter 1: Introduction___ 1

A. Spirochetes _ 2

Unique Characteristics__ 3

Research Emphasis —— 4

B. Lyme Disease

A History of Lyme Disease __ 4

Passage of Lyme disease _ـ 5

Clinical Symptoms and Treatment of Lyme Disease ___ 6

C. Borrelia burgdorferi _

D. Swine Dysentery

A Disease of Economic Concern for Food Production 14

Diagnosis of swine dysentery

Identification of Brachyspira hyodysenteriae __ 15

E. Brachyspira hyodysenteriae __ 16

F. Motility and disease. 17

Motility as a Virulence Factor in Other Bacteria __ 17

Motility as a virulence Factor in Spirochetes___ 19

G. Spirochete Motility

PFs Rotate 19

A Model for Motility in B. burgdorferi 20

Translational Modes_rat 21

Flagella Rotation _— 21

Asymmetrical PF Rotation__ 26

H. PFs Influence Cell Morphology _ 27

I. External Flagella Structure and Composition.

Basal Body__ 28

Hook 29

Filament_ 29

J. Periplasmic Flagella Structure __ 32

Basal body __ 32

Hook 32

Complex Filaments 33

K. Complex Flagella Filaments __ 36

Multiple Flagellins in Other Bacteria___ 36

Multiple Flagellins in Spirochetes _ 39 
Advantages of Multiple Flagellins

Arrangement of FlaB Subunits in the PF Core

L. Regulation of Flagella Synthesis__ 41

Salmonella 41

Caulobacter 42

Vibrio 43

Other Spirochetes _ 44

B. burgdorferi 44

M. Research Objectives $\quad 45$

Elucidate PF Synthesis Regulation in B. burgdorferi

Investigate the FlgE Ladder in B. burgdorferi $\longrightarrow 45$

Generate monoclonal antibodies for use in determining location of FlaB subunits in $B$.

hyodysenteriae _ 46

N. Literature Cited _ 47

Chapter 2. The decrease in FlaA observed in a flaB mutant of Borrelia burgdorferi occurs post-transcriptionally__ 60

Abstract__ 61

Introduction

Materials and Methods

Results _ 71

Discussion __ 84

References __ 91

Chapter 3. Post-transcriptional flagellin regulation in a periplasmic flagellar hook mutant of Borrelia burgdorferi ___ 97

Abstract__ 98

Introduction $\quad 99$

Materials and Methods

Results__ 110

Discussion _ 127

References _ 135

Chapter 4: The high molecular weight ladder of the periplasmic flagella hook in Borrelia burgdorferi. _ 140

Abstract__ 141

Introduction _ 143

Materials and Methods

Results _ 149

Discussion _ 157 
Chapter 5. Production of Monoclonal Antibodies to the Periplasmic Flagella Subunits of the Spirochete Brachyspira hyodysenteriae 165

Abstract 166

Introduction 167

Materials and Methods 170

Results 173

Discussion 183

References 188

Chapter 6: General Discussion 191

References 204

Appendix 206

Curriculum Vitae 207 


\section{$\underline{\text { Table of Figures }}$}

Chapter 1

Figure1. Spirochete morphology and ultrastructure.

Figure 2. Circular and linear plasmids in B. burgdorferi.

Figure 3. Motility and chemotaxis genes on the linear chromosome of $B$. burgdorferi.

Figure 4. Flagellum basal body and motor in enteric bacteria.......................23

Figure 5. PF Rotation and Swimming modes of $B$. burgdorferi. .......................25

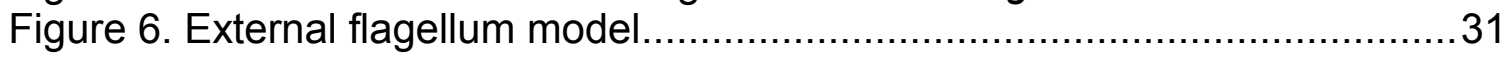

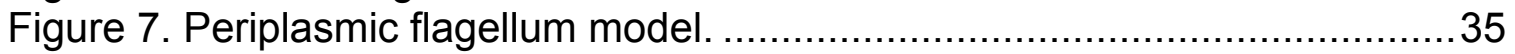

Figure 8: Arrangement of flagellins on filaments from different bacteria. ...........38

\section{Chapter 2}

Figure 1. Immunoprecipitation of FlaA with monoclonal H9724 anti-FlaB .........72

Figure 2. Quantitative Western blot analysis of PF protein levels in wild type $B$. burgdorferi.

Figure 3. Effect of flaB::kan on other motility and chemotaxis proteins.............77

Figure 4. Effect of flaB::kan on proteins encoded by genes in the flaA operon. .79

Figure 5. Detection and quantification of relative amounts of flaA transcript in wild-type and MC-1 strain using real-time RT-PCR.

Figure 6. Degradation of FlaA.

\section{Chapter 3}

Figure 1. Map of kan insertion into flgE and selected PCR primers...............111

Figure 2. Southern blot analysis of SC-1 and wild type B31 ...................... 114

Figure 3. PCR analysis of SC-1 ...................................................... 114

Table 1. Primer sequences. .......................................................... 115

Figure 4. Western blot analysis of gene products from the flgB operon...........116

Figure 5. Cell motility and morphology in SC-1 ................................... 118

Figure 6 . Wesern blot analysis of PF filament proteins. ........................... 121

Figure 7. Western blot analysis of gene products downstream of flaA ........... 122

Figure 8. Quantitative RT-PCR of filament genes. ................................. 125

Figure 9. Protein degradation assay. ............................................. 126

\section{Chapter 4}

Figure 1. PF and hook-basal body isolation.

Figure 2. SDS-PAGE and Western blot of purified PFs with crude polyclonal

FlgE antiserum and affinity purified FlgE antibodies. ........................... 152

Figure 3. Western blot analysis of the PF hook.

Figure 4. Western blot of wild type and PF mutants with crude FlgE antiserum. 


\section{Chapter 5}

Figure 1. Lymphocytes producing antibodies reactive to FlaB proteins. ..........174

Figure 2. Hybridomas identified and monoclonal antibody specificity............. 175

Figure 3. Hybridomas subcloned for monoclonal antibody production............177

Figure 4. Monoclonal antibody specificity across a panel of PF mutants........178

Figure 5. Electron micrographs of $B$. hyodysenteriae whole cells and PF. .....180

Figure 6. Model of possible FlaB arrangement on a PF filament. 
Chapter 1: Introduction 


\section{A. Spirochetes}

Spirochetes comprise a unique and intriguing group of ancient bacteria that have found quite diverse niches to inhabit. Some spirochetes, including Leptospira biflexa, are found free living in nature, while others are found occupying various creatures. In the ocean, Cristispira inhabits the crystalline style of mollusks (21). On land, several spirochetes are found living in the guts of termites or ticks. One such organism is Borrelia burgdorferi, which also infects mammalian hosts and is the causative agent of the arthropod borne Lyme disease (21). In addition to $B$. burgdorferi, many other spirochetes are medically significant bacteria that invade and induce a variety of diseases in both humans and animals. For example, Treponema pallidum is responsible for causing syphilis, a sexually transmitted disease, while Treponema denticola is implicated in periodontal disease (21). Brachyspira species are implicated in diarrheal diseases in humans (B. pilosicoli) and cause swine dysentery ( $B$. hyodysenteriae) (79). Leptospirosis is caused by spirochetes (Leptospira) found in water contaminated with urine from infected animals (21). Clearly, spirochete species have adapted to live in diverse niches in nature.

Relatively little is known about spirochetes, as these bacteria are difficult to cultivate. Spirochetes are fastidious bacteria with unique growth requirements that must first be determined before maintaining these organisms outside of their natural environments. This has proven difficult, particularly in the case of the syphilis spirochete. The growth requirements of T. pallidum have not been elucidated, and as a result the syphilis spirochete has yet to be cultivated. 
Likewise, some oral spirochete species have been identified by DNA sequencing and by morphology, although attempts to maintain these species in pure culture have been unsuccessful. As a result, research on these spirochetes is progressing slowly.

\section{Unique Characteristics}

Spirochetes are unique among bacteria because they can be identified by morphology as well as by $16 \mathrm{~S}$ ribosomal RNA gene sequence analysis $(4,54$, 106). Morphologically, spirochetes have a characteristic flat-wave or helical shape, which is advantageous for cell motility under certain conditions $(21,41$, 42). Spirochete motility is made possible by one or more periplasmic flagella (PFs) attached subterminally at each end of the protoplasmic cell cylinder that may or may not overlap at the center of the cell $(22,37,54,79)$. Unlike the external flagella of other bacteria, the spirochete PFs are located between the cell cylinder and an outer membrane sheath (54). Their unique motility allows for extremely efficient swimming through viscous gel-like media, such as that found in connective tissue, which inhibit the motility of other bacteria $(6,42,43,59,63$, $65,111,118)$. This characteristic may be advantageous for traversing cell layers and for dissemination throughout the host during infection. Evidence continues to mount for the essential role of PFs and motility as virulence factors for pathogenic spirochetes. 


\section{Research Emphasis}

The research emphasis in our lab is to understand how motility and chemotaxis contribute to virulence in pathogenic spirochetes. Our long term goals are to determine the roles of motility and chemotaxis in the disease process. To meet these goals, we must first understand the mechanisms of motility and chemotaxis in spirochetes. Our approach involves constructing and characterizing mutants deficient in periplasmic flagella or chemotaxis genes that impact the motility of a spirochete. Two pathogenic spirochetes of particular importance to our research are Borrelia burgdorferi and Brachyspira hyodysenteriae.

\section{B. Lyme Disease}

\section{A History of Lyme Disease}

$B$. burgdorferi is the causative agent of Lyme disease, the most prevalent arthropod-borne disease in the Unites States today $(1,128)$. Diseases caused by $B$. burgdorferi have been reported in countries around the world with isolates that fall into three categories: B. afzelli, B. garinii, and B. burgdorferi sensu stricto (128). Only B. burgdorferi sensu stricto has been identified in causing Lyme disease in the United States. Interest in the mysterious disease was piqued in 1975 when the Connecticut Health Department contacted Allen Steere regarding a woman's concern about an unusually large proportion of children diagnosed with juvenile rheumatoid arthritis in her neighborhood (46). By the early 1980's, the same spirochetes that Willy Burgdorfer identified in the gut of Ixodes scapularis ticks had been isolated from Lyme disease patients (46). The disease 
itself was named after the town in which it was first described in the United States, Old Lyme, CT. Today, the northeastern portion of the Unites States still reports the highest incidence of Lyme disease annually, with the most cases clustered in Connecticut, Rhode Island, New Jersey, Pennsylvania, and New York (1). Although Lyme disease may be under-reported, the number of diagnosed cases has risen steadily over the last decade to 23,763 reported in 2002 (www.cdc.gov).

\section{Passage of Lyme disease}

A close correlation exists between disease incidence and geographic location that results from the method by which spirochetes are transmitted between vector and host. A mammalian reservoir for $B$. burgdorferi is the white footed mouse, Peromyscus leucopus (46). In addition, B. burgdorferi exists in the guts of $I$. scapularis ticks in the eastern United States, and in the guts of $I$. pacificus on the west coast $(92,128)$. The transmission of spirochetes between mammal and tick follows the life cycle of the tick. In the spring, eggs hatch and develop into larva that will take a blood meal from small mammals, such as the white footed mouse $(46,92)$. If this mammal harbors $B$. burgdorferi, then the spirochetes are passed to the tick with a high probability during its meal. The tick then develops into its nymph stage by the following spring. At this time, it will take another blood meal and pass on the spirochetes to infect a new mammalian host, either another mouse or a larger host such as the white-tailed deer or human $(46,92,128)$. It is during the nymph phase of development that ticks are 
most likely to transmit Lyme disease spirochetes to humans, perhaps because their bite is painless or they are too small to be detected and removed within the first 12-72 hours of feeding $(92,128)$ (www.cdc.gov). By the end of summer, the ticks have molted into adults that will mate and lay eggs. The eggs hatch during the following spring and the cycle continues (46). In accordance with the life cycle and feeding habits of the tick, most Lyme disease cases are reported in the summer months (www.cdc.gov).

\section{Clinical Symptoms and Treatment of Lyme Disease}

Unlike the white-footed mouse, humans are not a practical reservoir for $B$. burgdorferi. Rather, humans represent an accidental host. In the human, Lyme disease is a multi-systemic infection presenting a variety of clinical manifestations that progress in stages. Stage I is early infection when there are general influenza-like symptoms including fever, headache, and fatigue (128). During this first stage of infection, the characteristic "bull's eye rash", erythema migrans, is evident in up to $50 \%$ of patients within several weeks $(46,92,128)$. This type of rash is associated with the localized spreading of spirochetes from the site of inoculation (128). Symptoms, particularly in the early stages of disease, are transient and usually subside with antibiotic treatment. Stage II is early dissemination when the spirochetes have spread away from the site of inoculation to other tissues. B. burgdorferi has been found to affect musculoskeletal tissue by inducing arthritis, the nervous system by causing conditions such as Bell's palsy, and less commonly cardiac tissue leading to 
arrhythmia (128). If left untreated, Lyme disease progresses to Stage III where the symptoms experienced are chronic and may involve autoimmune responses (128). Symptoms at Stage III may appear years after the initial tick bite.

Approximately $60 \%$ of untreated patients may experience arthritis in larger joints, such as the knee, during the late stage of infection (128). In most cases, the treatment for Lyme disease consists of a 2-4 week regimen of antibiotics, which commonly includes doxycycline or amoxycillin (128).

\section{Borrelia burgdorferi}

B. burgdorferi is a Gram negative, microaerophilic bacterium with characteristic flat wave morphology $(21,42,128)$. Motility is provided by $7-11$ PFs subterminally attached near each end of the cell $(4,21)$. These PFs extend inward, wrap around the cell cylinder, and overlap at the center of the cell (21). An outer membrane sheath surrounds the cell cylinder and PFs (Figure 1) (4, 21). The cells are approximately $10-30 \mu \mathrm{m}$ in length and $0.33 \mu \mathrm{m}$ in diameter (4, 128). 
$\mathbf{A}$

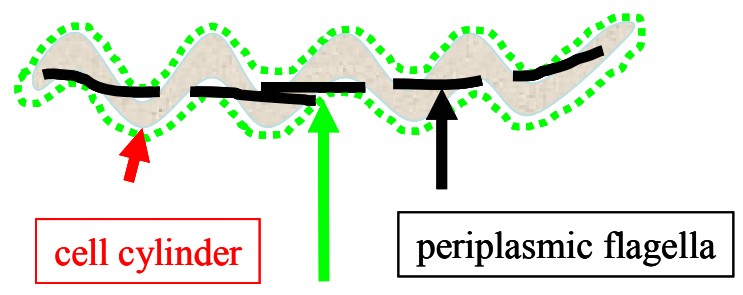

outer membrane sheath
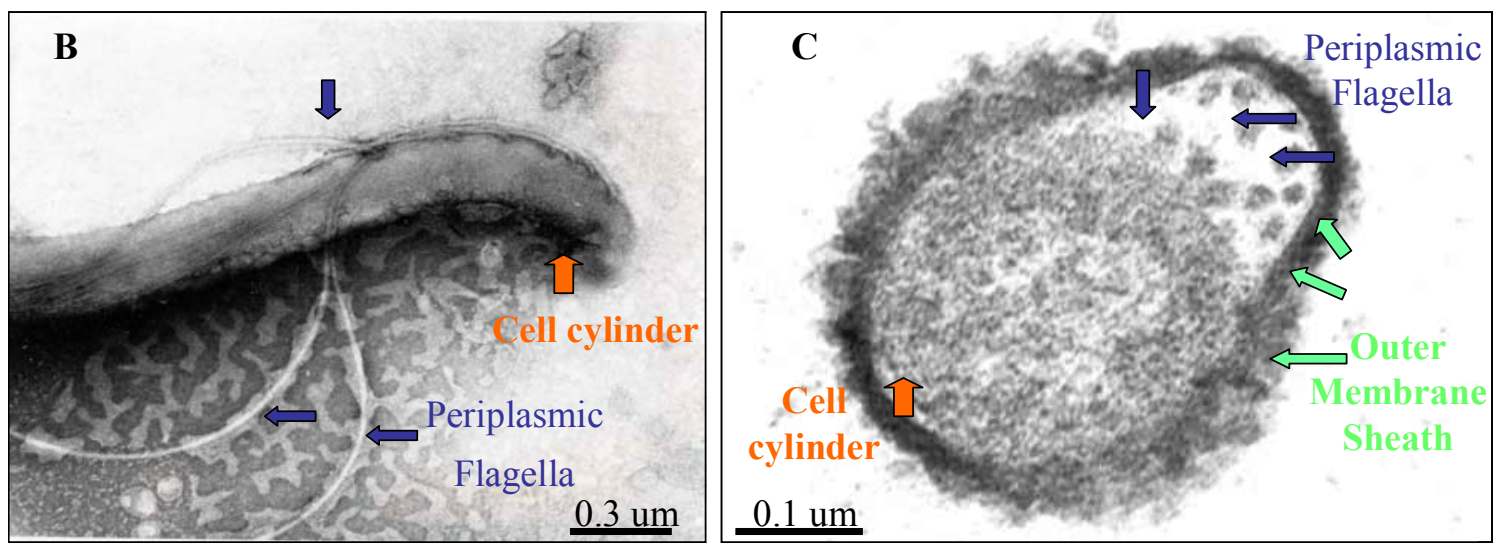

Figure1. Spirochete morphology and ultrastructure.

A. Model of spirochete ultrastructure. The cell cylinder, periplasmic flagella, and outer membrane sheath are denoted by arrows (21). B. Micrograph of B. burgdorferi with outer membrane removed. C. Cross-section of $B$. burgdorferi with cell cylinder, periplasmic flagella, and outer membrane sheath denoted by arrows. Reprinted, with permission, from the Annual Review of Genetics, Volume 36 @ 2002 by Annual Reviews www.annualreviews.org 
B. burgdorferi is cultured in a very rich media, Barbour-Stoenner-Kelley (BSK) media. These spirochetes thrive at $32-34^{\circ} \mathrm{C}$ in a $3 \%$ carbon dioxide environment and achieve a doubling time of approximately 10 hours. Although the doubling time becomes markedly longer, these spirochetes are viable for long periods of time when maintained at lower temperatures, $21-23^{\circ} \mathrm{C}$ for example, that might be encountered in the midgut of a tick. Interestingly, the protein profile of $B$. burgdorferi, particularly the outer membrane surface proteins (Osp), varies when this organism is grown in different hosts, or when cultured at different temperatures and environmental conditions $(103,127,128)$. By altering their protein expression, these cells can meet the demands of living in a new host environment when passed between the tick and mammal.

The Lyme disease spirochete has a genome consisting of a single linear chromosome of approximately 0.9 megabase, and multiple linear and circular plasmids (Figures 2 and 3) (34). B. burgdorferi isolates vary in regard to the number and type of plasmids present in their genomes. Furthermore, isolates may lose expression of some genes or lose entire plasmids during long-term in vitro cultivation (125). Also during long-term culture, B. burgdorferi exhibits reduced infectivity, suggesting that genes encoded on the discarded plasmids play a role in virulence for this organism $(96,126,142)$. One plasmid in particular, Ip25, was shown to be important for virulence, particularly with respect to a single gene encoded on that plasmid. Complementation of the BBE22 gene in a strain that lacks the Ip25 plasmid restored infectivity in a mouse model 
indicating that the BBE22 gene encoding a nicotinamidase on the Ip25 plasmid plays a direct role in virulence (112). Similar infectivity studies involving the presence or absence of select plasmids indicate that certain plasmids have important roles in B. burgdorferi virulence (76). Motility and chemotaxis are also considered to be virulence factors for spirochetes and their role in B. burgdorferi is under investigation. The motility and chemotaxis genes are grouped into operons located throughout the chromosome (Figure 3) $(34,36,39)$. The importance of motility and chemotaxis to $B$. burgdorferi is evident, as this bacterium allocates more than $6 \%$ of its entire chromosome to those genes. 


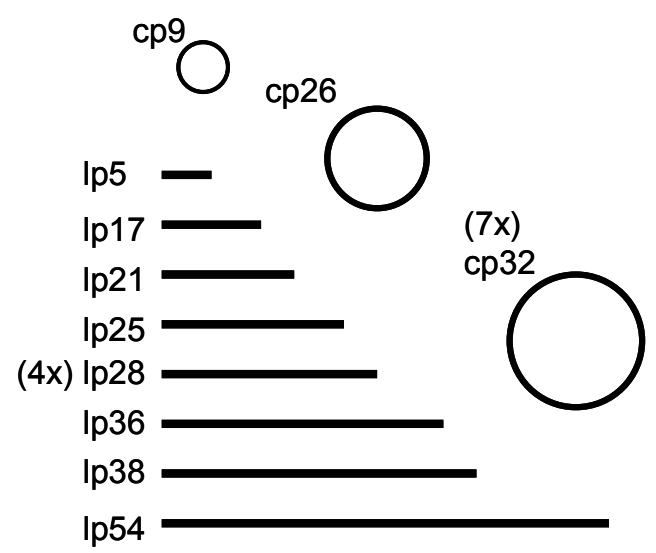

Figure 2. Circular and linear plasmids in B. burgdorferi.

The numbers following a circular (cp) or linear plasmid designation (Ip) indicates the size of the plasmid in kilobases. The numbers in parentheses indicate that there are multiple plasmids of a given size. Adapted from Casjens (19) 


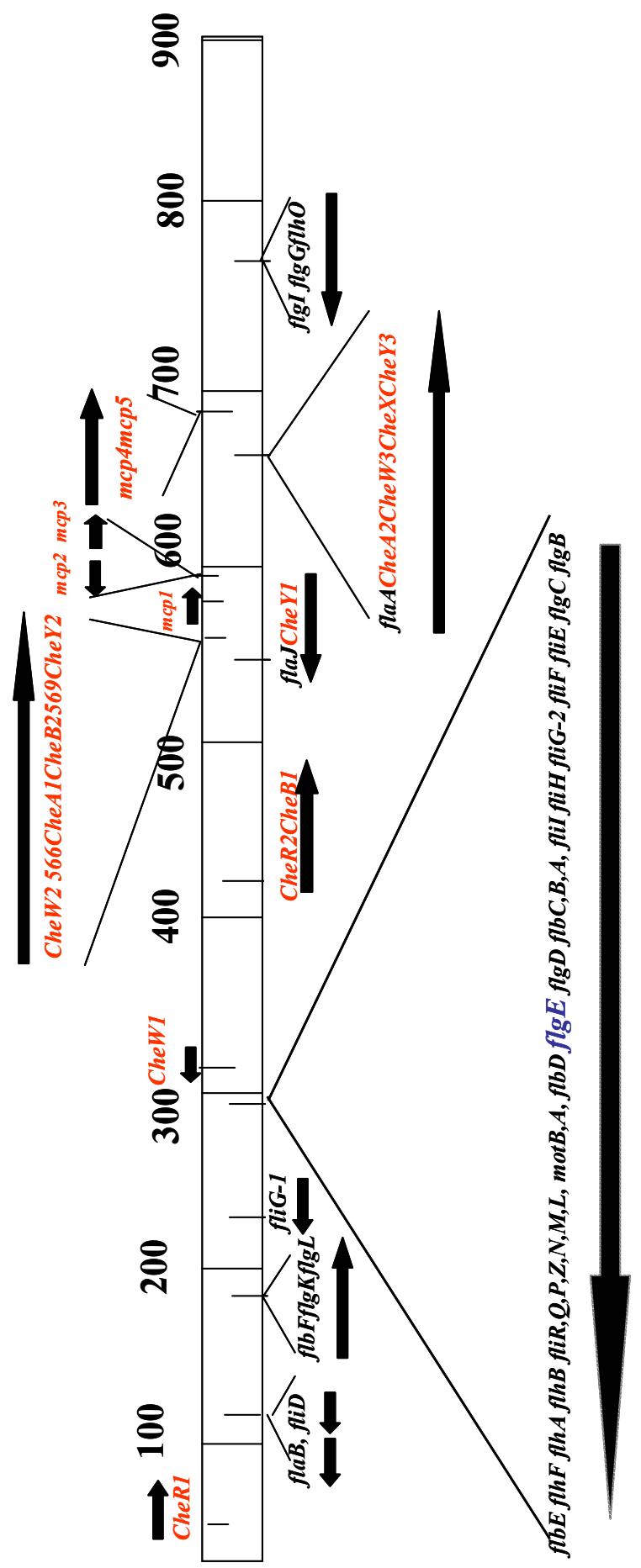

Figure 3. Motility and chemotaxis genes on the linear chromosome of $B$. burgdorferi.

Motility genes are shown in black, chemotaxis genes are shown in red. Arrows indicate the direction of transcription for motility and chemotaxis genes and operons $(38,79)$. Reprinted, with permission, from the Journal of Bacteriology Volume 179 @ 1979 by American Society for Microbiology. 
Ten years ago, electroporation mediated transformation of B. burgdorferi demonstrated that homologous recombination of the DNA gyrase gene, gyrB, was able to confer coumermycin resistance in these cells $(17,122)$. Subsequently, the mutated gry $B$ gene was used as a selectable marker to inactivate specifically targeted genes by allelic exchange mutagenesis (115). In addition to coumermycin resistance, selectable markers are available that encode resistance to kanamycin, streptomycin, gentamicin, and erythromycin $(12,30,33,123)$. The kanamycin resistance cassette has been successfully used for inactivating motility and chemotaxis genes by investigators in our laboratory $(77,88)$. As the ability to genetically manipulate $B$. burgdorferi continues to progress, researchers are finding that the antibiotic resistance cassettes are useful not only for gene inactivation, but also as selectable markers for colony isolation during complementation (124). Complementing a mutation requires adding back a functional copy of the mutated gene. The organism must regain wild type characteristics to conclusively demonstrate the function of that given gene. In the recent past, a number of shuttle vectors have been designed to deliver functional genes on a plasmid for complementation. An advantage of the shuttle vector is that it is able to replicate in Escherichia coli, for easy genetic manipulation, and also in $B$. burgdorferi, to express the functional gene of interest $(123,133)$. The antibiotic resistance cassettes mentioned above provide a variety of selectable markers for isolating complemented strains on the background of other antibiotic resistances. Gene complementation in $B$. 
burgdorferi is still relatively new and difficult to achieve. Complementation of the motility mutant MC-1 was only achieved in cis after a recombination event occurred between the complementation shuttle vector and chromosome (124). Complementation of a motility mutant in trans from a shuttle vector has not yet been reported in $B$. burgdorferi.

\section{Swine Dysentery}

\section{A Disease of Economic Concern for Food Production}

$B$. hyodysenteriae is responsible for causing the mucohemorrhagic diarrheal disease in pigs known as swine dysentery. Swine dysentery is passed through herds by fecal contamination of food, equipment, and other animals in close proximity to the swine (98)(www.thepigsite.com). Although its incubation period in a host is one to two weeks, $B$. hyodysenteriae can be carried and shed for many weeks from infected animals (www.thepigsite.com). Overcrowding and the introduction of new infected or carrier pigs increase disease in the herd (www.thepigsite.com). Swine dysentery affects mostly weanling to finishing pigs weighing $15-70 \mathrm{~kg}$, but is also seen in adults and piglets (98)(www.thepigsite.com). The disease is characterized by inflammation of the large intestine, severe epithelial damage, and increasingly bloody diarrhea as the disease progresses (32). Virulent $B$. hyodysenteriae have a strong and specific attraction to porcine intestinal mucin, and are commonly found within the crypts of Lieberkuhn or in close association with necrotic gut epithelium $(62,86)$. Death resulting from swine dysentery only occurs in up to $25 \%$ of infected pigs (129). However, the survivors grow poorly, maintain only minimal protection against re- 
infection, and can be asymptomatic carriers of the spirochetes that continue to infect others in the herd (129). Swine dysentery has an economic impact on swine production as there are high costs involved in antibiotic treatment of the herd, morbidity and mortality of pigs, and low feed conversion efficiency (98)(www.thepigsite.com).

\section{Diagnosis of swine dysentery}

The diagnosis of swine dysentery requires a positive identification of cultured spirochetes isolated from gut contents or feces of an infected pig. The pathogenic $B$. hyodysenteriae is strongly beta-hemolytic, which differentiates it from non-pathogenic intestinal spirochetes, such as Brachyspira innocens and Brachyspira intermedia $(32,97,98)$. More recent methods of PCR have been used to identify intestinal spirochetes down to the species level $(32,114)$. Thus, it is now possible to rapidly differentiate $B$. hyodysenteriae from other pathogenic spirochetes, including Brachyspira pilosicoli that may be present and cause a less severe porcine intestinal spirochetosis $(114,135,136)$.

\section{Identification of Brachyspira hyodysenteriae}

Although swine dysentery was described in 1921, B. hyodysenteriae wasn't identified as the etiological agent until 1972 (48). At the time, a large spirochete isolated from feces of infected pigs was named Treponema hyodysenteriae based on its morphology, anaerobic nature, and the ability to cause clinical swine dysentery when fed to pigs in pure or mixed cultures (40, 
48). This spirochete was initially considered to be Treponema, yet it was notably different from other treponemes. Upon further examination, this organism was renamed Serpulina hyodysenteriae, and more recently, Brachyspira hyodysenteriae based on genome analysis $(100,130)$.

\section{E. Brachyspira hyodysenteriae}

$B$. hyodysenteriae is a Gram negative, oxygen tolerant anaerobic spirochete with a loosely coiled helical morphology $(47,97)$. Motility is provided by bundles of 10-13 periplasmic flagella (PFs) that are subterminally attached near both ends of the cell cylinder $(18,50)$. These PFs extend inward along the cell cylinder and overlap near the center of the cell $(18,50)$. Like other spirochetes, an outer membrane sheath surrounds the cell cylinder and PFs. The whole cell is approximately $5-10 \mu \mathrm{m}$ in length and 0.3-0.8 $\mu \mathrm{m}$ in diameter (50). Outside of the animal, $B$. hyodysenteriae can survive for approximately six weeks at temperatures below $10^{\circ} \mathrm{C}$, but only one week at temperatures above $25^{\circ} \mathrm{C}$ in a moist environment (www.thepigsite.com)(129). In vitro culture occurs in Brain Heart Infusion (BHI) media supplemented with $10 \%$ fetal bovine serum at $34-$ $36^{\circ} \mathrm{C}$ in an environment of $3-5 \%$ oxygen. Under these conditions, $B$. hyodysenteriae can reach a doubling time of approximately 5 hours (72).

The genome of $B$. hyodysenteriae is composed of a $3.2 \mathrm{Mb}$ circular chromosome (143). Although there is low level resistance to kanamycin, this antibiotic resistance marker is used to inactivate targeted genes (134). A chloramphenicol resistance cassette (cat) was also developed as a selective 
marker for gene inactivation in B. hyodysenteriae (116). Both of these markers have been utilized, either alone or in combination, for the inactivation of motility genes $(78,79,116)$. More recently, the need for further selectable markers prompted the development of a coumermycin resistant cassette for $B$. hyodysenteriae (131). In addition to targeted manipulation of genes in this spirochete species, use of the coumermycin resistance marker has contributed to the understanding of a natural genetic transfer system involving $\mathrm{VSH}-1$ (131). VSH-1, virus of Serpulina hyodysenteriae, is a bacteriophage inducible by mitomycin $C$ that transduces random $7.5 \mathrm{~kb}$ fragments of DNA in $B$. hyodysenteriae (56). VSH-1 was reported as the first evidence of genetic transduction in spirochetes (56). The gene encoding the VSH-1 major head protein, vsp38, has recently been detected in several Brachyspira species, but not in species from Borrelia, Treponema, Leptospira, or Spirocheta $(56,132)$.

\section{F. Motility and disease.}

\section{Motility as a Virulence Factor in Other Bacteria}

Flagella and motility are known to play a role in colonization and pathogenicity. For example, flagella from Pseudomonas aeruginosa contribute to pathogenicity by binding to tissue or by providing motility to the cells.

Examination of wild type, motility mutant, and revertant cell colonization in a burned-mouse model implicates flagella as a virulence factor for $P$. aeruginosa (87). Animal studies reveal that immunization with flagellin antigens or treatment with antisera against flagellin will confer protection against subsequent challenge or prevent host invasion after colonization $(28,28,49,49,99)$. In addition, 
motility is a colonization factor for Campylobacter jejuni, a cause of human disease. Wild type $C$. jejuni readily colonizes the intestinal tracts in a mouse model, whereas flagella and motility mutants fail to successfully colonize (93). Similar results are obtained from experiments where nonflagellated mutants of Campylobacter coli fail to colonize a rabbit model and confer minimal protection against subsequent challenge when used as a vaccine (107). Furthermore, flagella adherence of Vibrio cholerae to intestinal mucosa likely aids in colonization of the host gut and subsequently contributes to its pathogenesis ( 3 , 3 , 44). Attridge and Rowley examined the adherence capabilities of antibodyimmobilized wild type and non-flagellated $V$. cholerae (3). The results suggest that it is not motility per se that promotes attachment to host cells, rather flagella themselves specifically play a role in adherence regardless of motile behavior. Conversely, based on examination of both mouse and rabbit models of $V$. cholerae pathogenicity, Richardson suggests that cell motility contributes more to colonization of the host than does the flagella structure itself (113). More recent results from wild type, non-motile, and nonchemotactic mutants suggest that a smooth swimming cell is required for $V$. cholerae infection. Smooth swimming in $V$. cholerae is achieved when rotation of its polar flagellum is biased in a counterclockwise direction, which occurs during a transient non-chemotactic state when the bacteria is passed between hosts (16). Taken together, the evidence above indicates that flagella and motility contribute to the pathogenesis of these organisms. 


\section{Motility as a virulence Factor in Spirochetes}

Motility contributes to the pathogenesis of spirochetes as well as other bacteria. Spirochetes are uniquely adept at traversing viscous gel-like media, like that found in host connective tissue or mucosal surfaces $(6,43,43,59,59$, $63,63,65,65,111,111,118,118)$. When motility is lost, as in a flagella-less mutant of $B$. burgdorferi, the ability of that spirochete to penetrate a cell monolayer is reduced (120). Motility mutants of $B$. hyodysenteriae that have altered flagellin genes, including single flaA and flaB1 mutants and the double mutant lacking both FlaA and FlaB1, have reduced motility and a reduced capacity to colonize the gut in a mouse model compared to wild type $B$. hyodysenteriae $(61,117)$. Taken together, motility is considered a virulence factor for pathogenic spirochetes.

\section{G. Spirochete Motility}

\section{PFs Rotate}

Early studies of spirochete PFs suggested that these structures might be involved in cell motility based on their similarity to flagella from other bacteria. Observations in Leptospira motility mutants documented that, in addition to a loss of translational and non-translational motility, these cells had altered PFs and lost the morphological spiral and hook shaped cell ends (15). Shortly thereafter, Paster and Canale-Parola analyzed motility mutants that lacked PFs and proposed that PFs were the structures responsible for providing motility to Spirocheta aurantia (105). Direct evidence for the rotation of PFs came by analyzing the motions of PFs protruding from Treponema phagedenis. The PF 
protrusions were observed rotating both clockwise (CW) and counterclockwise (CCW) (22). Similar results were found for Treponema denticola and $B$. burgdorferi indicating that the rotation of PFs provides motility to multiple spirochete species (22).

\section{A Model for Motility in B. burgdorferi}

Although it has been determined that spirochete motility is provided by the rotation of PFs, the mechanism by which PF rotation led to translational movement was unclear. Basic examination of the swimming behavior in $B$. burgdorferi reveals that these spirochetes swim in a planar waveform, rather than a corkscrew fashion (42). Planar waves originate at the front leading end of the cell and propagate toward the back of the cell. It is noteworthy that the wavelength of the cell body and the PF helix were identical (41). Since the PFs are in close proximity to the cell cylinder, it was hypothesized that the PFs influenced the shape of the entire cell during translation. The PFs themselves are left-handed in space, yet the PF bundles wrap around the cell body in a right handed fashion in B. burgdorferi (41). A motility model predicts that counterclockwise rotation of the PF bundles, as viewed from the back of the cell, causes the backward moving flat waves that push the whole cell forward. At the same time, the cell rolls about its body axis in a clockwise direction to counter balance the flat wave movement (79). 


\section{Translational Modes}

Externally flagellated enteric bacteria move from one location to another by a series of runs in one direction and tumbles, in which forward translation stops and the bacterium changes direction. In enteric bacteria, the CCW rotation of flagella causes the formation of a tight bundle that acts as a propeller to produce a forward run (137). To tumble and change direction, one or more flagella must reverse the rotation to $\mathrm{CW}$ causing the bundle to dissociate and the cell to reorient in space (137). Rotation of the flagella CCW again allows the cell to run in its new direction (21). As bacteria translate, they combine runs and tumbles to move in a biased random walk toward more favorable environments. The direction of flagella rotation is determined by a signaling cascade of chemotaxis proteins (Che) in response to environmental factors. Chemotaxis occurs as the methyl accepting chemotaxis proteins (Mcp) bind substrate and signal for the autophosphorlyation of the sensor histidine kinase CheA. CheA will pass the phosphate group to CheY, which interacts with the flagellar switch complex to increase the probability of $\mathrm{CW}$ rotation and cell tumbling. In the absence of a chemotactic signal or during adaptation to an attractant, the default state of flagellar rotation is CCW and the cell runs (21).

\section{Flagella Rotation}

The switch and motor complexes are responsible for providing flagellar rotation. These structures are located at the site of flagellum insertion in the cell membrane. In the switch complex of enteric bacteria, FliG mainly functions in flagella rotation and interacts with FliM to generate torque. FliM, which is 
involved in directional switching, is also associated with FliN tetramers that stabilize the flagellar switch complex $(27,66)$. FliG is bound to the MS ring component, FliF, and interacts with the motor protein MotA. MotA is located around the MS ring in complexes with MotB (66).

Eight Mot complexes act as the stator and anchor the motor in the cell membrane $(7,9)$. In addition, Mot complexes provide sites for transmembrane proton channels to translocate ions (27). The process of flagellum rotation is driven by the proton motive force, or ionic gradient, across the inner membrane rather than an expenditure of ATP (27). Flagellar motors can reach impressive rotation frequencies of $18,000 \mathrm{rpm}$ in Salmonella species to over $100,000 \mathrm{rpm}$ in other bacterial species (27). Although many models for the mechanism of flagella rotation have been proposed, evidence continues to support a model for the interaction of motor proteins described as a ratchet action that turns the flagellum in a number of discrete steps (66). 


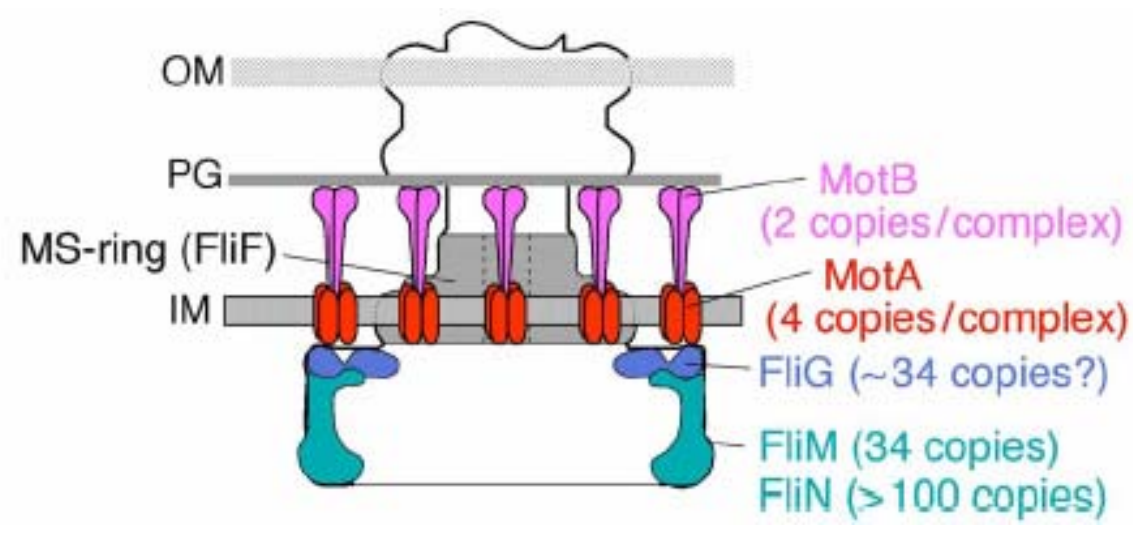

Figure 4. Flagellum basal body and motor in enteric bacteria

Basal body rings and their location in the bacterial membranes are indicated. Mot complexes and FliG are the major players in this model of the possible arrangement of motor components in a basal body (8). Reprinted with permission from $D$. Blair. 
Spirochete motility is more complex than that of externally flagellated bacteria for a number of reasons. First, spirochete PFs are physically located inside the cell and cannot act as a propeller to provide motility. Second, spirochetes have bundles of PFs at opposite ends of the cell to coordinate for translation. Spirochetes have three motility modes: run, stop, and flex. A run can occur in either direction where the PFs at the leading end are rotating CCW, and those at the posterior end are rotating CW. A stop occurs when the cell ceases to run, followed by a run in the same or opposite direction. A flex is described as both PF bundles rotating in the same direction, which results in the cell bending at the center (21). Since the default state of flagellar rotation in enteric bacteria that have left-handed flagella is $\mathrm{CCW}$, one may infer that, in the absence of environmental signals, the spirochete would always have its two cell ends trying to run in opposite directions at the same time. This scenario is not conducive to translational motility for spirochetes. Therefore, the model explaining the observed motility modes asserts that there must be asymmetrical rotation of the PF bundles during translation, as viewed from the center of the cell looking toward the ends. 


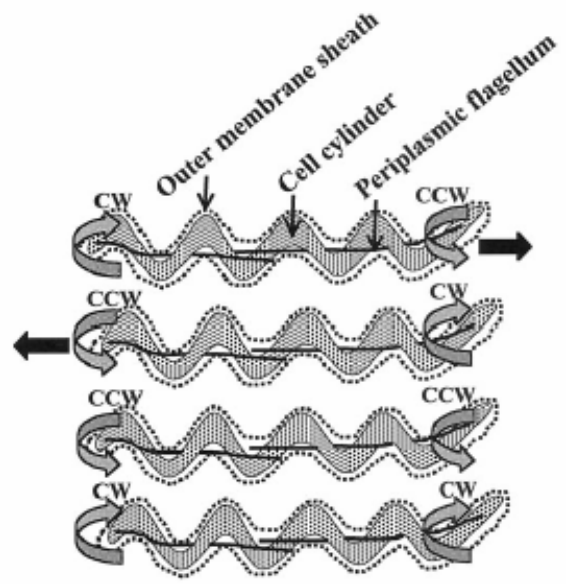

Figure 5. PF Rotation and Swimming modes of B. burgdorferi.

Cellular components of the spirochete are as indicated. Dark arrows indicate the direction of cell swimming. Curved arrows indicate the direction of PF rotation, clockwise (CW) or counterclockwise (CCW), as viewed from the center of the cell looking toward the PF. The first two models show translating cells. The last two models show flexing cells $(21,77)$. Reprinted, with permission, from the Annual Review of Genetics, Volume $36 @ 2002$ by Annual Reviews www.annualreviews.org. 


\section{Asymmetrical PF Rotation}

How spirochetes coordinate asymmetrical PF rotation for translation is still under investigation. There are two possible explanations for this phenomenon. First, there may be inherent differences in the default state of PF rotation at opposite ends of the cell. Motors controlling the direction of PF rotation at one end of the cell may be physically different than those at the other end. This scenario involves one PF bundle in the default state rotating $\mathrm{CCW}$, while the other rotates $\mathrm{CW}$, such that a cell will constantly run in the absence of environmental signals. It is noteworthy that $B$. burgdorferi and some treponemes have two different copies of fliG genes encoding a switch protein for the PF motor (21). FliG is a component of the switch complex, and is involved in flagellum rotation (77). The role of multiple fliG genes in asymmetrical PF rotation is still under investigation. Another possible explanation for asymmetrical PF rotation is that there may be differences in the chemotaxis signaling cascade that direct PFs at one end to rotate CCW, while those at the other end rotate CW. The large size of a spirochete implies that there should be a delay in relaying chemotactic signals due to diffusion from one end of the cell all the way to the other. However, a change in swimming direction can occur quite fast. Perhaps both ends of the cell have chemotactic machinery to independently sense and react to the environment simultaneously. In contrast to enteric bacteria, $B$. burgdorferi has multiple copies of chemotaxis genes, including 2 che $A$, 3 cheY, and 2 cheW, whose roles are currently under investigation $(35,77)$. Furthermore, there are several open reading frames 
located in motility operons whose functions are undetermined. One such gene is cheX, which is not apparent in many bacteria and may therefore be involved in asymmetrical PF rotation. These genes may play some role in coordinating asymmetrical PF rotation in $B$. burgdorferi.

\section{H. PFs Influence Cell Morphology}

The close proximity of PFs to the cell cylinder and outer membrane sheath suggest that the rigid flagella can influence the cell shape of spirochetes. The two PFs, one at each end, of Leptospiraceae do not overlap at the center of the cell and are shown to influence the shape of cell ends. It has been noted that Leptospira lacking PFs are helical with straight ends, whereas the wild type have irregularly shaped cell ends (119). In translating cells, the leading anterior end is spiral-shaped while the posterior end is hook-shaped (79). As the cell reverses its swimming direction, the cell ends change shape accordingly. In T. denticola, the presence of PFs gives the wild type cell an irregular twisted morphology with planar and helical regions (119). In contrast, a flgE mutant that lacked PFs had a right-handed helical morphology along the length of the cell (119). A more striking example of PF influence on cell morphology is documented in $B$. burgdorferi where the PFs perform a skeletal role along the length of the cell in addition to providing motility. MC-1, the targeted mutant lacking the major filament flagellin FlaB, was shown to be rod-shaped in stark contrast to the wavelike wild type cells (88). Complementation of MC-1 restored motility and wild type morphology to the cells (124). 


\section{External Flagella Structure and Composition.}

External flagella are composed of multiple proteins making up the basal body, hook, and filament structures (Figure 6).

\section{Basal Body}

The basal body component of a flagellum is composed of multiple structural proteins embedded within the cell membranes (for a recent review see Kojima 2004). Construction of the basal body begins with an MS ring composed of FliF in the cytoplasmic membrane (66). The MS ring contacts the rod and C ring at the cytoplasmic membrane (66). The $\mathrm{C}$ ring includes FliN and FliM proteins that are involved in the switch complex (66). The flagellar rod, composed of $\mathrm{FlgB}, \mathrm{FlgC}, \mathrm{FlgF}$, and $\mathrm{FlgG}$, is assembled and extends through both the inner and outer membranes (66). The rod cap, FlgJ, is a muramidase that can break down peptidoglycan in the cell wall during flagellar assembly (66). The $P$ ring is the next ring to form around the rod and it resides in the peptidoglycan layer (21). A final ring, the $L$ ring, assembles around the rod and anchors in the outer membrane of Gram negative bacteria $(21,66)$. In addition to structural proteins, multiple proteins including peptidoglycan hydrolases and chaperones for subunit polymerization are involved in construction of the basal body-rod complex $(66,93)$. The basal body also serves as a site for congregation of various proteins to coordinate motor function, described previously. 


\section{Hook}

Distal to the rod of the basal body is the hook structure. The flagellar hook is a polymer composed of protein subunits encoded by the flgE gene, and acts as a flexible coupling for the flagellar filament to the basal body $(11,58,64,91)$. FlgE monomers vary in size depending on species from approximately $42 \mathrm{kDa}$ in Escherichia coli to $76 \mathrm{kDa}$ in Helicobacter pylori (www.tigr.org). The hook monomers polymerize to form a hollow tube of approximately $55 \mathrm{~nm}$, measured in Salmonella enterica serovar Typhimurium (21). Although the C-terminus was found to be required for hook polymerization, the overall length of the hook is actually controlled by another protein, fliK $(85,121,121,138)$. Hook-associated proteins, HAP1 and HAP3, and flagellin monomers travel though the hollow hook structure for subsequent assembly (51-53). In addition, the hook indirectly plays a regulatory role in flagella synthesis that will be described in a later section.

\section{Filament}

Flagella filaments are formed distal to the hook, and upon rotation provide locomotion to the cell (121). Thousands of flagellin monomers exit a cell through the hook structure and polymerize at the end of a growing filament $(7,66)$. This activity is monitored by FliD, the filament capping protein that prevents flagellin from escaping into the medium $(21,57)$. FliD also aids in the addition of flagellin monomers onto the growing filament (57). Many bacteria have simple filaments composed of one type of flagellin monomer, FliC (21). However, bacteria including Caulobacter crescentus, Campylobacter coli, and Helicobacter pylori 
have complex flagella filaments composed of multiple flagellin proteins $(45,70$, 139). Complex filaments will be discussed later. 


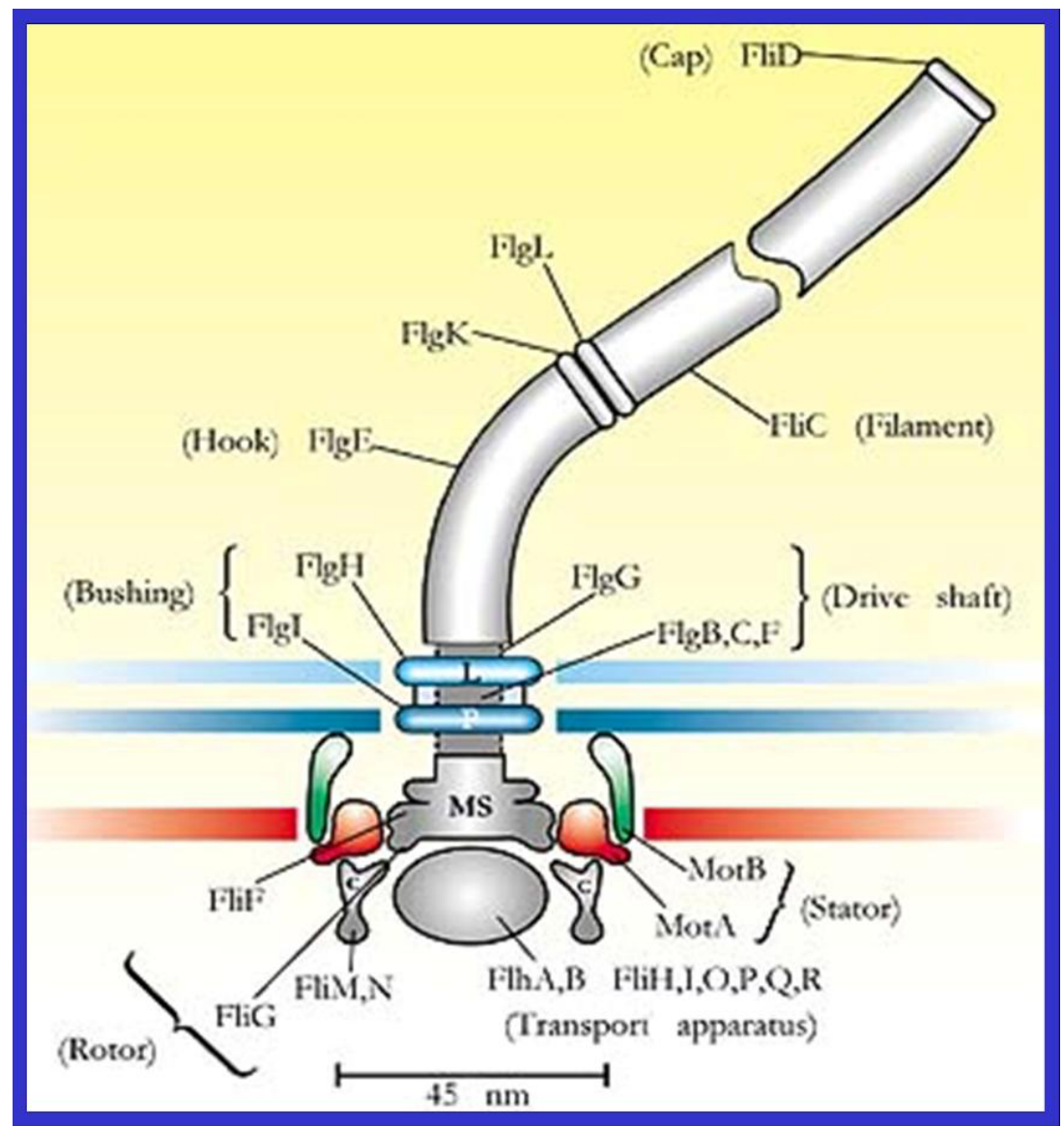

Figure 6. External flagellum model

Proteins that are involved in the construction and rotor function of external flagella from enteric bacteria are indicated (7). Reprinted with permission from Berg (Jan 2000) Physics Today pp24-29 @ 2000, American Institute of Physics. Also with permission from $\mathrm{H}$. Berg and D. DeRosier. 


\section{J. Periplasmic Flagella Structure}

Spirochete PFs are similar in composition to the flagella of other bacteria; however, these structures are located within the periplasmic space rather than external to the cell (Figure 7). Their location beneath the outer membrane sheath presents some difficult challenges for investigating the composition and rotation of these periplasmic flagella structures.

\section{Basal body}

Since PFs do not cross an outer membrane, it is not surprising that the gene encoding the $L$ ring of spirochete basal bodies is absent $(21,34)$. Therefore, one may infer that the basal body is anchored in and crosses only the cytoplasmic membrane in spirochetes. Interestingly, there are multiple fliG genes, encoding the switch protein of the motor in B. burgdorferi and T. pallidum $(34,35,79)$. The location and function of the different FliG proteins in PFs is unknown. Furthermore, $\operatorname{mot} A$ and $\operatorname{mot} B$ genes have been identified, but the location of Mot complexes near a flagellum has not yet been elucidated in spirochetes $(34,79)$.

\section{Hook}

The PF hook is similar in structure and function to the flagellar hook in other bacteria. The PF hook is encoded by the flgE gene that shows sequence similarity to flgE genes in other bacteria $(58,82)$. The PF hook varies in size depending on the spirochete. Specifically, the PF hook has an apparent mass of 
approximately $40 \mathrm{kDa}$ reported in $B$. burgdorferi and $55 \mathrm{kDa}$ in $T$. phagedenis as determined by SDS-PAGE $(58,82)$. Furthermore, the PF hook in $T$. phagedenis was determined to be on average $69 \mathrm{~nm}$ in length and $23 \mathrm{~nm}$ in diameter (82). In B. burgdorferi, the PF hook measures $58 \mathrm{~nm}$ in length and $17 \mathrm{~nm}$ in diameter (71). An interesting feature of PF hooks from T. phagedenis and T. denticola is the high molecular weight protein ladder that appears in SDS-PAGE and Western blot $(24,82)$. Such a ladder pattern indicates that subunit cross-linking occurs in the PF hook structure. The importance of hook cross-linking in spirochetes is unknown. It has been reported by Jwang that $B$. burgdorferi hook subunits are not cross-linked due to the absence of a high molecular weight banding pattern (58). However, preliminary evidence in our laboratory indicates otherwise, and the possibility of hook subunit cross-linking in $B$. burgdorferi is currently under investigation (see Chapter 4).

\section{Complex Filaments}

Spirochetes have complex PF filaments composed of several different proteins from two classes of flagellins: FlaA and FlaB. Spirochetes have one or two FlaA proteins that form a sheath around the filament core and influence the shape of the PF in some species $(10,13,26,69,78,79)$. The FlaA proteins range in size from $37-39 \mathrm{kDa}$ by SDS-PAGE $(14,23,95)$. Based on the location of an amino-terminal cleavage sequence, FlaA proteins are thought to be secreted to the periplasmic space by a sec-A dependent pathway, where they then interact with the FlaB filament core $(89,95)$. The filament core is composed 
of one to four FlaB proteins that range in size from $33-41 \mathrm{kDa}$ and are encoded by separate $f l a B$ genes $(20,35,79,104)$. Since the flaB genes lack an aminoterminal signal sequence, it is thought that they are secreted through the PF hook structure of the Type III secretion system, similar to flagellin of other bacteria (95). The flaB genes share considerable homology with each other and are strongly conserved across spirochete species (94). Accordingly, the FlaB proteins are highly antigenically related and cross-react with antisera to other FlaB proteins, even those of different spirochetes $(13,81,95)$. 


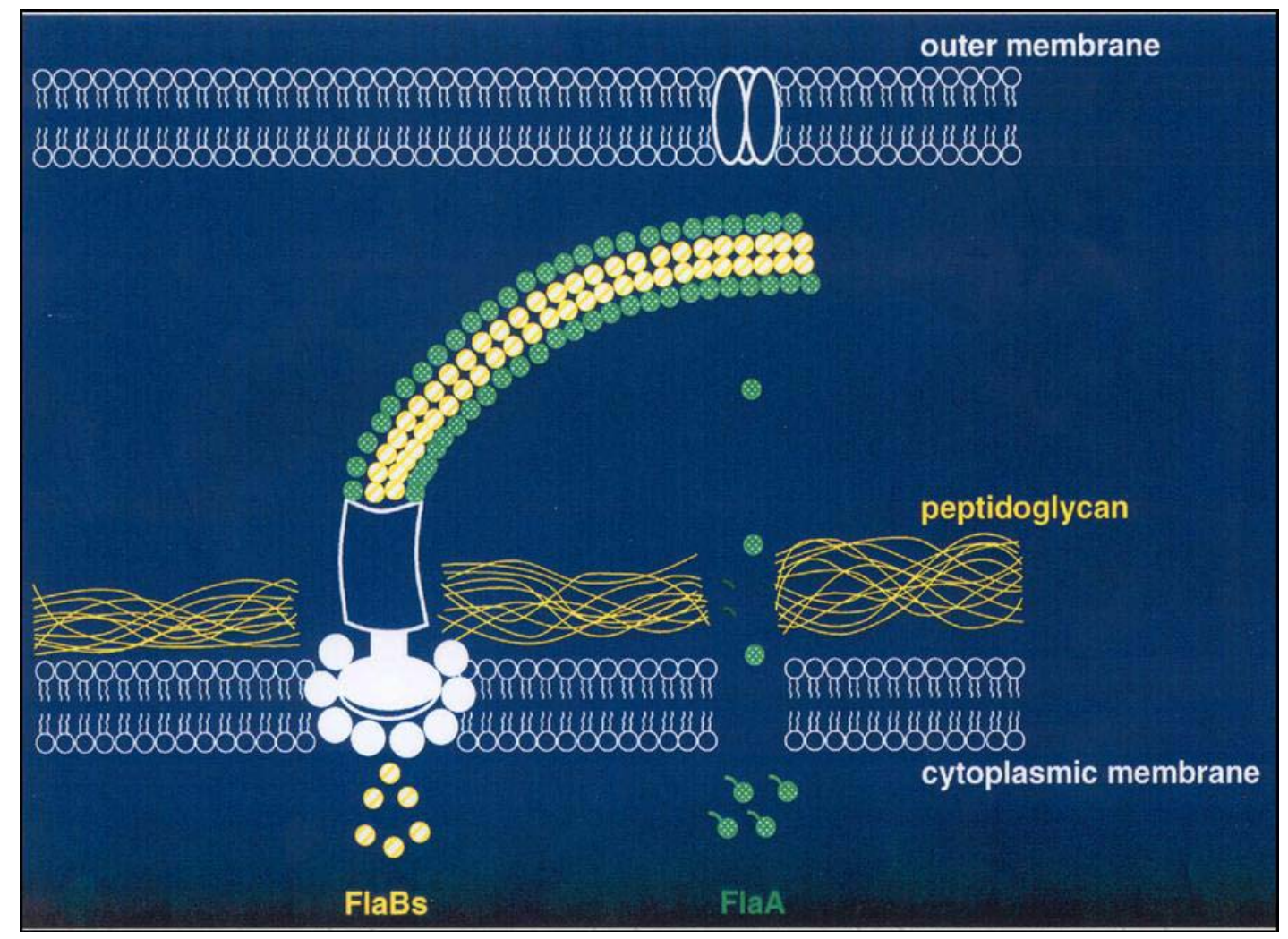

Figure 7. Periplasmic flagellum model.

PFs are anchored in the cytoplamic membrane and remain contained in the periplasmic space. The PF filament is composed of a core made of one or more FlaB proteins (yellow circles) surrounded by a FlaA sheath (green circles) (21, 23). Reprinted, with permission, from the Annual Review of Genetics, Volume 36 (C) 2002 by Annual Reviews www.annualreviews.org. 


\section{K. Complex Flagella Filaments}

\section{Multiple Flagellins in Other Bacteria}

In many bacteria, the external flagella filaments are composed of a polymer of FliC protein subunits. In contrast, several other bacteria including Caulobacter crescentus, Rhizobium meliloti, Campylobacter coli, and Helicobacter pylori have complex flagellar filaments consisting of multiple flagellin protein species. C. crescentus has at least three flagellin proteins in its filament, and the arrangement of these flagellins was investigated by immunoelectron microscopy (29). A 29kD FljJ protein is located proximal to the hook, followed by a section containing the $27 \mathrm{kD}$ FljL protein (29). Beyond the FljL region, increasing amounts of $25 \mathrm{kD}$ flagellin monomers assemble toward the distal region of the filament (29). The $25 \mathrm{kD}$ flagellin monomers are thought to be products of one or more flagellin genes including $f l j K, f l j M, f l j N$, and $f l j O$ (31). Specific regions of a filament are occupied by different flagellin proteins in $R$. meliloti. The FlaB flagellin is located near the hook and the FlaA flagellin assembles at the distal portion of the filament $(73,108,109,109)$. Whereas FlaA subunits are not assembled without FlaB, flaA mutants could form truncated filaments from assembly of FlaB subunits (109). Mutant analysis reveals that double mutants do not produce flagella filaments and are non-motile (109). Like the flagella filament made by $R$. meliloti, H. pylori also produces flagellar filaments from two flagellin proteins (70). FlaB is found near the hook and subunits of FlaA comprise the remainder of the filament (70). The flagellin proteins of $H$. pylori are antigenically similar to those of $C$. coli based on crossreactivity of antisera (70). C. coli also produce flagellar filaments composed of 
FlaA and FlaB; both of which are necessary for full motility (45). However, unlike C. crescentus, R. meliloei, and H. pylori, the flagellin proteins of C. coli are distributed along the length of the filament rather than segregated to specific regions on the flagella $(29,45,73,109)$. These examples illustrate that there are several patterns for building complex flagellar filaments from multiple flagellin proteins to allow for optimal cell motility (Figure 8). 


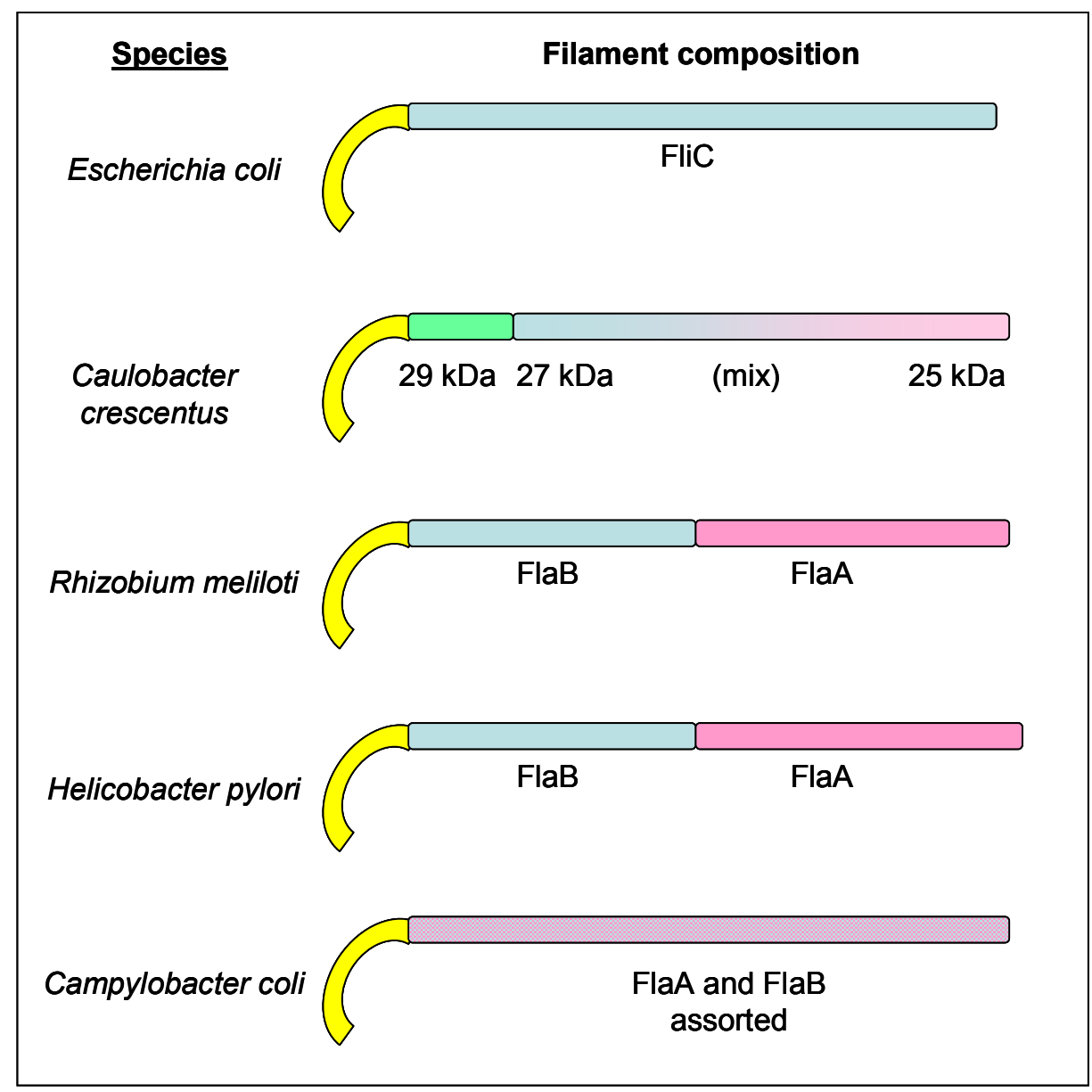

Figure 8: Arrangement of flagellins on filaments from different bacteria.

The flagellar hook is shown in yellow. Flagellar filaments are depicted with different colored bars indicating the location and arrangement of flagellin proteins. In Caulobacter crescentus, the $29 \mathrm{kDa}$ flagellin is FljJ, the $27 \mathrm{kDa}$ flagellin is FljL and the $25 \mathrm{kDa}$ flagellin is FljK. 


\section{Multiple Flagellins in Spirochetes}

B. burgdorferi has a PF filament core polymerized from a single FlaB protein species of $41 \mathrm{kDa}$, and a FlaA protein of $39 \mathrm{kDa}$ that associates with FlaB (89). Other spirochetes, including those in Spirochaeta, Leptospira, Treponema, and Brachyspira, have PF filament cores composed of multiple species of antigenically related FlaB proteins ranging from $33 \mathrm{kDa}$ to $39 \mathrm{kDa}$, and FlaA sheath proteins $(13,13,68,68,69,79,81,81,95,95)$. The FlaB proteins of Treponema are similar in size and cross-react with antisera (95). Based on sequence analysis and antigenic cross-reactivity, the PF filament core of Treponema is similar to that of Brachyspira (67). Although each individual FlaB subunit is transcribed from its own gene, the three FlaB proteins of Brachyspira share a high degree of homology and cross-react with FlaB antibodies, including those raised against Treponema (68). Currently, the arrangement of FlaB subunits along the complex spirochete PF filament is unknown. Considering the similarities between species, Brachyspira makes a convenient model system for studying PF composition of Treponema, specifically T. pallidum, as this pathogenic syphilis spirochete cannot be cultured in vitro.

\section{Advantages of Multiple Flagellins}

The reason behind multiple FlaB proteins in B. hyodysenteriae, and other spirochetes, is not clear. One possibility is that motility is so important for the survival of spirochetes, that they maintain multiple flagellin genes to compensate in the event that one is lost. For example, when the single flaB gene is 
inactivated in B. burgdorferi, these cells completely lack motility (88).

Furthermore, the levels of FlaA were greatly reduced (89). In contrast, when one of the flaB genes is inactivated in $B$. hyodysenteriae, these cells are still capable of motility $(79,116)$. Single and double mutants were generated in $B$. hyodysenteriae using allelic exchange techniques with kanamycin and chloramphenicol resistence cassettes $(78,116)$. The inactivation of a particular flaB gene does not negatively influence the expression of the other flagellins in this system. Based on mutant analysis, it appears that FlaB proteins may have overlapping function, as single mutants still produce PF filaments capable of supporting motility $(79,116)$. Interestingly, the flaB1flaB2 double mutant of $B$. hyodysenteriae lacks motility and does not appear to assemble PFs $(78,79$, 117). These results indicate that either FlaB1 or FlaB2 is necessary to form a PF filament capable of supporting motility in B. hyodysenteriae.

\section{Arrangement of FlaB Subunits in the PF Core}

Thus far, the location of FlaB1, FlaB2, and FlaB3 along the PF filament core has not been determined in B. hyodysenteriae or in Treponema species. Immunoelectron microscopy has been a successful technique for visualizing the arrangement of flagellins in bacteria including C. crescentus (29). Due to the high cross-reactivity of all three FlaB proteins, we propose to isolate monoclonal antibodies reactive to individual FlaB proteins. With these subunit specific antibodies, one can employ immunoelectron microscopy using gold beads to visualize the architecture of the PF filament in B. hyodysenteriae. Determining 
the structural organization of the PF filament core may provide evidence regarding the necessity for multiple FlaB proteins in spirochetes.

\section{Regulation of Flagella Synthesis}

Flagella synthesis is a finely orchestrated succession of motility gene expression and protein assembly. In many bacteria, flagella are synthesized in response to environmental factors (84). In contrast, B. burgdorferi PFs appear to be constitutively produced due to the lack of flagella specific transcriptional regulation (21). Flagella have been calculated to comprise approximately $10 \%$ of total protein in multiflagellate cells $(68,89)$. Accordingly, the production and maintenance of flagella is energetically expensive to a cell. The numerous proteins involved in the synthesis of flagella require tight regulation, usually by a cascade of transcriptional events involving flagella-specific promoters described later in this section. For many bacterial species, completion of the basal bodyhook structure acts as a general assembly checkpoint in the regulation of flagella synthesis, allowing the flagellar hook to play an indirect role in regulating flagella synthesis. In this case, the flagellin genes are controlled transcriptionally in response to the state of hook completion. In the following subsections, various methods of regulating flagella synthesis will be described in the context of different bacteria.

\section{Salmonella}

In S. enterica, flagella synthesis is regulated by a cascade of transcriptional events involving the ordered expression of class I genes, class II 
genes, and class III genes. Expression of class I genes fIhCD, which comprise the master operon, directs the transcription of class II genes (83). Class II genes are those that encode structural proteins involved with the early stages of flagella synthesis and regulatory proteins controlling the expression of late stage class III flagella and chemotaxis genes (60). Among the class II regulatory genes are fliA, encoding the flagella specific transcription factor sigma-28, and flgM, encoding the anti-sigma-28 factor $(60,101)$. Prior to and during hook synthesis, the activity of $\sigma^{28}$ is inhibited by FlgM that accumulates in the cytoplasm (25). Upon completion of the hook structure, FlgM is excreted through a channel within the hook, leaving sigma-28 to initiate the transcription of Class III late genes, including flagellin $(25,55,74,75,101,102)$. If an intact hook is not formed or is not functional, then FlgM cannot escape from the cytoplasm and remains associated with sigma-28 to prevent the transcription of flagellin genes (60). As a result, the bacterium cannot assemble a filament in the absence of hook. In this case, the flagellin genes are controlled transcriptionally in response to the state of hook completion. Hence, the flagellar hook acts as a regulatory structure.

\section{Caulobacter}

Although S. enterica flagella regulation and synthesis has been extensively studied and previously described, not all bacterial species regulate their flagella synthesis in the same manner. Caulobacter crescentus regulates its polar flagellum synthesis by a hierarchy of four classes of motility genes in 
respose to cell cycle (90). The Class I product CtrA is required for expression of Class II genes (141). Of the Class II gene products, including those for the basal body MS ring and regulatory genes, sigma 54 acts as a flagella specific transcription factor to initiate the transcription of Class III and Class IV genes (2, $90,140,141)$. In addition, assembly of the early basal body structure from Class II gene products is necessary to activate FlbD, which is required for Class III expression $(5,90,141)$. Class III genes encode proteins necessary to complete the basal body rings, rod, and hook structures $(90,141)$. Construction of the hook structure is a regulatory checkpoint for flagellum synthesis that is needed for continuation of Class IV flagellin gene expression $(2,141)$. In addition to transcriptional cascade control, the $25 \mathrm{kDa}$ FljK and $27 \mathrm{kDa}$ FljL flagellins are regulated by different post-transcriptional control mechanisms $(2,141)$.

\section{Vibrio}

Another variation on the theme of regulating flagella synthesis occurs in Vibrio cholerae. V. cholerae regulates the synthesis of its flagella by a cascade through four classes of motility genes controlled by both sigma 54 and sigma 28 transcriptional activators (110). In this way, the regulation of flagella synthesis includes aspects from both S. enterica and C. crescentus. The Class I product FIrA acts with sigma 54 to transcribe Class II genes, including genes for the MS ring, chemotaxis, and regulatory genes for FliA (sigma 28) and FIrC (110). The Class III gene transcription for basal body, hook, and core flagellin is initiated by sigma 54 and its activator FIrC (110). The expression of the Class IV genes is 
dependent on the activity of sigma 28 to complete flagella assembly (110).

Similar to $S$. enterica, completion of the basal body-hook structure acts as a general assembly checkpoint in the transcriptional regulation of flagella synthesis for these bacteria.

\section{Other Spirochetes}

Relatively little is known about the regulation of PF synthesis in spirochetes. The hierarchy of motility gene expression has not been as extensively studied as that in other bacteria. Flagellar hook mutants from the oral spirochete $T$. denticola, where the flgE gene was interrupted with an erythromycin resistance cassette, were found to be non-motile and lack PFs (80). Furthermore, flgE mutants produced minimal amounts of FlaA and FlaB proteins (119). These results indicate that a hook structure is necessary for the production of flagella filaments, and thus necessary to support motility in these bacteria. In terms of flagellin expression, sequence analysis of flagellin genes from various spirochetes revealed sigma-28 like promoters in Treponema species (flaB1, flaB2, and flaB3) and Brachyspira species (flaB1, flaB2). Furthermore, other flagellin genes have sigma-70 like promoters, including fla $A$ in T. pallidum, and flaA and flaB3 in B. hyodysenteriae (79).

\section{B. burgdorferi}

Although bacterial flagella are similar in structure, evidence indicates that the regulation of PF synthesis in $B$. burgdorferi differs from flagella synthesis in 
other species of bacteria. Evidently, the transcription factor sigma-28 and its anti-sigma factor FlgM are not involved in B. burgdorferi PF regulation. Specifically, FlgM and sigma-28 sequences have not been identified in $B$. burgdorferi $(34,34,79)$. Furthermore, analysis of the promoter regions for PF genes reveals sequences that are better suited to transcriptional activation by the general transcription factor sigma- 70 than by the flagella specific sigma-28 ( 37 , 79). Therefore, PF synthesis in B. burgdorferi does not follow the widely accepted models previously described for the transcriptional regulation of flagella synthesis.

\section{Research Objectives}

\section{Elucidate PF Synthesis Regulation in B. burgdorferi}

Currently, a regulatory role for the hook structure in PF synthesis has not been determined in $B$. burgdorferi. To begin understanding the system of PF regulation in this spirochete, we proposed to inactivate the PF hook gene. Analysis of the flgE mutant strain obtained would allow us to identify PF genes whose products are affected by the absence of the hook. In doing so, we can begin to break down the mechanism behind PF synthesis regulation in $B$. burgdorferi.

\section{Investigate the FIgE Ladder in B. burgdorferi}

The PF hook in Treponema phagedenis and Treponema denticola are thought to be covalently cross-linked based on evidence of a ladder of multiple high molecular weight bands reactive to FlgE antisera. Although it has been 
reported that the $B$. burgdorferi hook is not cross-linked, preliminary data from our lab indicates that the high molecular weight ladder of polypeptides is indeed present. We propose to further investigate the nature of the high molecular weight FlgE bands found in B. burgdorferi.

\section{Generate monoclonal antibodies for use in determining location of FlaB subunits in $B$. hyodysenteriae}

Brachyspira hyodysenteriae has three FlaB proteins making up the PF filament core, and a FlaA protein that forms a sheath around the filament core. The reason for multiple FlaB proteins in spirochetes is unknown. The three FlaB proteins share high homology and are antigenically related. As a result, it is common for all three FlaB proteins to cross-react with antisera. The first step to understanding the architechure of the PF filament in $B$. hyodysenteriae is to develop antibodies to the FlaB proteins which will not cross-react with the other flagellins. We proposed to generate monoclonal FlaB antibodies that could discriminate between the individual FlaB proteins. 


\section{N. Literature Cited}

1. 2004. Lyme Disease. MMWR Morb. Mortal. Wkly. Rep. 53:365-369.

2. Anderson, D. K. and A. Newton. 1997. Posttranscriptional regulation of Caulobacter flagellin genes by a late flagellum assembly checkpoint. J. Bacteriol. 179:2281-2288.

3. Attridge, S. R. and D. Rowley. 1983. The role of the flagellum in the adherence of Vibrio cholerae. J. Infect. Dis. 147:864-872.

4. Barbour, A. G. and S. F. Hayes. 1986. Biology of Borrelia species. Microbiol. Rev. 50:381-400.

5. Benson, A. K., J. Wu, and A. Newton. 1994. The role of FlbD in regulation of flagellar gene transcription in Caulobacter crescentus. Res. Microbiol. 145:420-430.

6. Berg, H. C. and L. Turner. 1979. Movement of microorganisms in viscous environments. Nature 278:349-51.

7. Berg, H. 2000. Motile Behavior of Bacteria. Physics Today Jan. 2000:2429.

8. Blair, D. F. 2003. Flagellar movement driven by proton translocation. FEBS Lett. 545:86-95.

9. Blair, D. F. and H. C. Berg. 1988. Restoration of torque in defective flagellar motors. Science 242:1678-1681.

10. Blanco, D. R., C. I. Champion, J. N. Miller, and M. A. Lovett. 1988. Antigenic and structural characterization of Treponema pallidum (Nichols strain) endoflagella. Infect. Immun. 56:168-175.

11. Bonifield, H. R., S. Yamaguchi, and K. T. Hughes. 2000. The flagellar hook protein, FlgE, of Salmonella enterica serovar typhimurium is posttranscriptionally regulated in response to the stage of flagellar assembly. J. Bacteriol. 182:4044-50.

12. Bono, J. L., A. F. Elias, J. J. Kupko, B. Stevenson, K. Tilly, and P. Rosa. 2000. Efficient targeted mutagenesis in Borrelia burgdorferi. J. Bacteriol. 182:2445-52.

13. Brahamsha, B. and E. P. Greenberg. 1988. Biochemical and cytological analysis of the complex periplasmic flagella from Spirochaeta aurantia. J. Bacteriol. 170:4023-4032. 
14. Brahamsha, B. and E. P. Greenberg. 1989. Cloning and sequence analysis of flaA, a gene encoding a Spirochaeta aurantia flagellar filament surface antigen. J. Bacteriol. 171:1692-1697.

15. Bromley, D. B. and N. W. Charon. 1979. Axial filament involvement in the motility of Leptospira interrogans. J. Bacteriol. 137:1406-12.

16. Butler, S. M. and A. Camilli. 2004. Both chemotaxis and net motility greatly influence the infectivity of Vibrio cholerae. Proc. Natl. Acad. Sci. USA 101:5018-5023.

17. Cabello, F. C., M. L. Sartakova, and E. Y. Dobrikova. 2001. Genetic manipulation of spirochetes--light at the end of the tunnel. Trends. Microbiol. 9:245-8.

18. Canale-Parola, E. 1978. Motility and chemotaxis of spirochetes. Annu. Rev. Microbiol. 32:69-99.

19. Casjens, S., N. Palmer, R. van Vugt, W. M. Huang, B. Stevenson, P. Rosa, R. Lathigra, G. Sutton, J. Peterson, R. J. Dodson, D. Haft, E. Hickey, M. Gwinn, O. White, and C. M. Fraser. 2000. A bacterial genome in flux: the twelve linear and nine circular extrachromosomal DNAs in an infectious isolate of the Lyme disease spirochete Borrelia burgdorferi. Mol. Microbiol. 35:490-516.

20. Champion, C. I., J. N. Miller, M. A. Lovett, and D. R. Blanco. 1990. Cloning, sequencing, and expression of two class B endoflagellar genes of Treponema pallidum subsp. pallidum encoding the 34.5- and 31.0kilodalton proteins. Infect. Immun. 58:1697-1704.

21. Charon, N. W. and S. F. Goldstein. 2002. Genetics of motility and chemotaxis of a facinating group of bacteria: The Spirochetes. Annu. Rev. Genet. 36:47-73.

22. Charon, N. W., S. F. Goldstein, S. M. Block, K. Curci, J. D. Ruby, J. A. Kreiling, and R. J. Limberger. 1992. Morphology and dynamics of protruding spirochete periplasmic flagella. J. Bacteriol. 174:832-40.

23. Charon, N. W., E. P. Greenberg, M. B. Koopman, and R. J. Limberger. 1992. Spirochete chemotaxis, motility, and the structure of the spirochetal periplasmic flagella. Res. Microbiol. 143:597-603.

24. Chi, B., R. J. Limberger, and H. K. Kuramitsu. 2002. Complementation of a Treponema denticola flgE mutant with a novel coumermycin A1resistant T. denticola shuttle vector system. Infect. Immun. 70:2233-2237. 
25. Chilcott, G. S. and K. T. Hughes. 2000. Coupling of flagellar gene expression to flagellar assembly in Salmonella enterica serovar typhimurium and Escherichia coli. Microbiol. Mol. Biol. Rev. 64:694-708.

26. Cockayne, A., M. J. Bailey, and C. W. Penn. 1987. Analysis of sheath and core structures of the axial filament of Treponema pallidum. J. Gen. Microbiol. 133 (Pt 6):1397-1407.

27. DeRosier, D. J. 1998. The turn of the screw: the bacterial flagellar motor. Cell 93:17-20.

28. Drake, D. and T. C. Montie. 1987. Protection against Pseudomonas aeruginosa infection by passive transfer of anti-flagellar serum. Can. J. Microbiol. 33:755-763.

29. Driks, A., R. Bryan, L. Shapiro, and D. J. DeRosier. 1989. The organization of the Caulobacter crescentus flagellar filament. J. Mol. Biol. 206:627-636.

30. Elias, A. F., P. E. Stewart, D. Grimm, M. J. Caimano, C. H. Eggers, K. Tilly, J. L. Bono, D. R. Akins, J. D. Radolf, T. G. Schwan, and P. Rosa. 2002. Clonal polymorphism of Borrelia burgdorferi strain B31 Ml: implications for mutagenesis in an infectious strain background. Infect. Immun. 70:2139-50.

31. Ely, B., T. W. Ely, W. B. Crymes, Jr., and S. A. Minnich. 2000. A family of six flagellin genes contributes to the Caulobacter crescentus flagellar filament. J. Bacteriol. 182:5001-5004.

32. Fellstrom, C., B. Pettersson, J. Thomson, A. Gunnarsson, M. Persson, and K. E. Johansson. 1997. Identification of Serpulina species associated with porcine colitis by biochemical analysis and PCR. J. Clin. Microbiol. 35:462-467.

33. Frank, K. L., S. F. Bundle, M. E. Kresge, C. H. Eggers, and D. S. Samuels. 2003. aadA confers streptomycin resistance in Borrelia burgdorferi. J. Bacteriol. 185:6723-7.

34. Fraser, C. M., S. Casjens, W. M. Huang, G. G. Sutton, R. Clayton, R. Lathigra, O. White, K. A. Ketchum, R. Dodson, E. K. Hickey, M. Gwinn, B. Dougherty, J. F. Tomb, R. D. Fleischmann, D. Richardson, J. Peterson, A. R. Kerlavage, J. Quackenbush, S. Salzberg, M. Hanson, R. van Vugt, N. Palmer, M. D. Adams, J. Gocayne, J. C. Venter, and et al. 1997. Genomic sequence of a Lyme disease spirochaete, Borrelia burgdorferi. Nature 390:580-6.

35. Fraser, C. M., S. J. Norris, G. M. Weinstock, O. White, G. G. Sutton, R. Dodson, M. Gwinn, E. K. Hickey, R. Clayton, K. A. Ketchum, E. 
Sodergren, J. M. Hardham, M. P. McLeod, S. Salzberg, J. Peterson, H. Khalak, D. Richardson, J. K. Howell, M. Chidambaram, T. Utterback, L. McDonald, P. Artiach, C. Bowman, M. D. Cotton, J. C. Venter, and et al. 1998. Complete genome sequence of Treponema pallidum, the syphilis spirochete. Science 281:375-88.

36. Ge, Y. and N. W. Charon. 1997. Molecular characterization of a flagellar/chemotaxis opernon in the spriochete Borrelia burgdorferi. FEMS Microbiol. Lett. 152:425-431.

37. Ge, Y., I. G. Old, I. Saint Girons, and N. W. Charon. 1997. Molecular characterization of a large Borrelia burgdorferi motility operon which is initiated by a consensus sigma70 promoter. J. Bacteriol. 179:2289-99.

38. Ge, Y., I. G. Old, I. Saint Girons, and N. W. Charon. 1997. Molecular characterization of a large Borrelia burgdorferi motility operon which is initiated by a consensus sigma70 promoter. J. Bacteriol. 179:2289-99.

39. Ge, Y. and N. W. Charon. 1997. Identification of a large motility operon in Borrelia burgdorferi by semi-random PCR chromosome walking. Gene 189:195-201.

40. Glock, R. D. and D. L. Harris. 1972. Swine dysentery. II. Characterization of lesions in pigs inoculated with Treponema hyodysenteriae in pure and mixed culture. Vet. Med. Small Anim. Clin. 67:65-68.

41. Goldstein, S. F., K. F. Buttle, and N. W. Charon. 1996. Structural analysis of the Leptospiraceae and Borrelia burgdorferi by high-voltage electron microscopy. J. Bacteriol. 178:6539-45.

42. Goldstein, S. F., N. W. Charon, and J. A. Kreiling. 1994. Borrelia burgdorferi swims with a planar waveform similar to that of eukaryotic flagella. Proc. Natl. Acad. Sci. USA 91:3433-7.

43. Greenberg, E. P. and E. Canale-Parola. 1977. Relationship between cell coiling and motility of spirochetes in viscous environments. J. Bacteriol. 131:960-9.

44. Guentzel, M. N. and L. J. Berry. 1975. Motility as a virulence factor for Vibrio cholerae. Infect. Immun. 11:890-897.

45. Guerry, P., R. A. Alm, M. E. Power, S. M. Logan, and T. J. Trust. 1991. Role of two flagellin genes in Campylobacter motility. J. Bacteriol. 173:4757-4764.

46. Habicht, G. S., G. Beck, and J. L. Benach. 1987. Lyme disease. Sci. Am. 257:78-83. 
47. Harris, D. L. and R. D. Glock. 1972. Swine dysentery. J. Am. Vet. Med. Assoc. 160:561-565.

48. Harris, D. L., R. D. Glock, C. R. Christensen, and J. M. Kinyon. 1972. Inoculation of pigs with Treponema hyodysenteriae (new species) and reproduction $f$ the disease. Vet. Med. Small Anim. Clin. 67:61-64.

49. Holder, I. A., R. Wheeler, and T. C. Montie. 1982. Flagellar preparations from Pseudomonas aeruginosa: animal protection studies. Infect. Immun. 35:276-280.

50. Holt, S. C. 1978. Anatomy and chemistry of spirochetes. Microbiol. Rev. 42:114-60.

51. Homma, M., D. J. DeRosier, and R. M. Macnab. 1990. Flagellar hook and hook-associated proteins of Salmonella typhimurium and their relationship to other axial components of the flagellum. J. Mol. Biol. 213:819-832.

52. Homma, M. and T. lino. 1985. Locations of hook-associated proteins in flagellar structures of Salmonella typhimurium. J. Bacteriol. 162:183-189.

53. Homma, M., K. Kutsukake, and T. lino. 1985. Structural genes for flagellar hook-associated proteins in Salmonella typhimurium. J. Bacteriol. 163:464-471.

54. Hovind-Hougen, K. 1984. Ultrastructure of spirochetes isolated from Ixodes ricinus and Ixodes dammini. Yale J. Biol. Med. 57:543-8.

55. Hughes, K. T., K. L. Gillen, M. J. Semon, and J. E. Karlinsey. 1993. Sensing structural intermediates in bacterial flagellar assembly by export of a negative regulator. Science 262:1277-80.

56. Humphrey, S. B., T. B. Stanton, N. S. Jensen, and R. L. Zuerner. 1997. Purification and characterization of VSH-1, a generalized transducing bacteriophage of Serpulina hyodysenteriae. J. Bacteriol. 179:323-329.

57. Ikeda, T., K. Oosawa, and H. Hotani. 1996. Self-assembly of the filament capping protein, FliD, of bacterial flagella into an annular structure. J. Mol. Biol. 259:679-686.

58. Jwang, B., P. Dewing, E. Fikrig, and R. A. Flavell. 1995. The hook protein of Borrelia burgdorferi, encoded by the flgE gene, is serologically recognized in Lyme disease. Clin. Diagn. Lab Immunol. 2:609-615.

59. Kaiser, G. E. and R. N. Doetsch. 1975. Letter: Enhanced translational motion of Leptospira in viscous environments. Nature 255:656-657. 
60. Karlinsey, J. E., S. Tanaka, V. Bettenworth, S. Yamaguchi, W. Boos, S. I. Aizawa, and K. T. Hughes. 2000. Completion of the hook-basal body complex of the Salmonella typhimurium flagellum is coupled to FlgM secretion and flic transcription. Mol. Microbiol. 37:1220-1231.

61. Kennedy, M. J., E. L. Rosey, and R. J. Yancey, Jr. 1997. Characterization of flaA- and flaB- mutants of Serpulina hyodysenteriae: both flagellin subunits, FlaA and FlaB, are necessary for full motility and intestinal colonization. FEMS Microbiol. Lett. 153:119-28.

62. Kennedy, M. J., D. K. Rosnick, R. G. Ulrich, and R. J. Yancey, Jr. 1988. Association of Treponema hyodysenteriae with porcine intestinal mucosa. J. Gen. Microbiol. 134 (Pt 6):1565-1576.

63. Kimsey, R. B. and A. Spielman. 1990. Motility of Lyme disease spirochetes in fluids as viscous as the extracellular matrix. J. Infect. Dis. 162:1205-8.

64. Kinsella, N., P. Guerry, J. Cooney, and T. J. Trust. 1997. The flgE gene of Campylobacter coli is under the control of the alternative sigma factor sigma54. J. Bacteriol. 179:4647-4653.

65. Klitorinos, A., P. Noble, R. Siboo, and E. C. Chan. 1993. Viscositydependent locomotion of oral spirochetes. Oral Microbiol. Immunol. 8:242244.

66. Kojima, S. and D. F. Blair. 2004. The bacterial flagellar motor: structure and function of a complex molecular machine. Int. Rev. Cytol. 233:93-134.

67. Koopman, M. B., E. Baats, O. S. de Leeuw, B. A. van der Zeijst, and J. G. Kusters. 1993. Molecular analysis of a flagellar core protein gene of Serpulina (Treponema) hyodysenteriae. J. Gen. Microbiol. 139 ( Pt 8):1701-1706.

68. Koopman, M. B., E. Baats, C. J. van Vorstenbosch, B. A. van der Zeijst, and J. G. Kusters. 1992. The periplasmic flagella of Serpulina (Treponema) hyodysenteriae are composed of two sheath proteins and three core proteins. J. Gen. Microbiol. 138 ( Pt 12):2697-2706.

69. Koopman, M. B., O. S. de Leeuw, B. M. van der Zeijst, and J. G. Kusters. 1992. Cloning and DNA sequence analysis of a Serpulina (Treponema) hyodysenteriae gene encoding a periplasmic flagellar sheath protein. Infect. Immun. 60:2920-2925.

70. Kostrzynska, M., J. D. Betts, J. W. Austin, and T. J. Trust. 1991. Identification, characterization, and spatial localization of two flagellin species in Helicobacter pylori flagella. J. Bacteriol. 173:937-946. 
71. Kreiling, J. A. 1994. Dissertation. West Virginia University.

72. Krieg, N. R. and J. G. Holt. 1984. Bergey's Manual of Systematic Bacteriology. Volume I. Section 1: Spirochetes. Williams \& Wilkins Baltimore, Maryland.

73. Krupski, G., R. Gotz, K. Ober, E. Pleier, and R. Schmitt. 1985. Structure of complex flagellar filaments in Rhizobium meliloti. J. Bacteriol. 162:361-366.

74. Kutsukake, K. and T. lino. 1994. Role of the FliA-FlgM regulatory system on the transcriptional control of the flagellar regulon and flagellar formation in Salmonella typhimurium. J. Bacteriol. 176:3598-605.

75. Kutsukake, K., S. lyoda, K. Ohnishi, and T. lino. 1994. Genetic and molecular analyses of the interaction between the flagellum-specific sigma and anti-sigma factors in Salmonella typhimurium. EMBO J. 13:45684576.

76. Lawrenz, M. B., R. M. Wooten, and S. J. Norris. 2004. Effects of vlsE complementation on the infectivity of Borrelia burgdorferi lacking the linear plasmid Ip28-1. Infect. Immun. 72:6577-6585.

77. Li, C., R. G. Bakker, M. A. Motaleb, M. L. Sartakova, F. C. Cabello, and N. W. Charon. 2002. Asymmetrical flagellar rotation in Borrelia burgdorferi nonchemotactic mutants. Proc. Natl. Acad. Sci. USA 99:6169-74.

78. Li, C., L. Corum, D. Morgan, E. L. Rosey, T. B. Stanton, and N. W. Charon. 2000. The spirochete FlaA periplasmic flagellar sheath protein impacts flagellar helicity. J. Bacteriol. 182:6698-706.

79. Li, C., A. Motaleb, M. Sal, S. F. Goldstein, and N. W. Charon. 2000. Spirochete periplasmic flagella and motility. J. Mol. Microbiol. Biotechnol. 2:345-54.

80. Li, H., J. Ruby, N. Charon, and H. Kuramitsu. 1996. Gene inactivation in the oral spirochete Treponema denticola: construction of an flgE mutant. J. Bacteriol. 178:3664-3667.

81. Limberger, R. J. and N. W. Charon. 1986. Treponema phagedenis has at least two proteins residing together on its periplasmic flagella. J. Bacteriol. 166:105-12.

82. Limberger, R. J., L. L. Slivienski, and W. A. Samsonoff. 1994. Genetic and biochemical analysis of the flagellar hook of Treponema phagedenis. J. Bacteriol. 176:3631-3637. 
83. Liu, X. and P. Matsumura. 1994. The FlhD/FlhC complex, a transcriptional activator of the Escherichia coli flagellar class II operons. J. Bacteriol. 176:7345-7351.

84. Macnab, R. M. 1996. Flagella and Motility, p. 123-145. In F. C. Neidhardt, R. Curtiss III, J. L. Ingraham, C. C. Lin, K. B. Low, B. Magasanik, W. S. Reznikoff, M. Riley, M. Schaechter, and H. E. Umbarger (eds.), Escherichia coli and Salmonella typhimurium:cellular and molecular biology. ASM Press, Washington D.C.

85. Makishima, S., K. Komoriya, S. Yamaguchi, and S. I. Aizawa. 2001. Length of the flagellar hook and the capacity of the type III export apparatus. Science 291:2411-2413.

86. Milner, J. A. and R. Sellwood. 1994. Chemotactic response to mucin by Serpulina hyodysenteriae and other porcine spirochetes: potential role in intestinal colonization. Infect. Immun. 62:4095-4099.

87. Montie, T. C., D. Doyle-Huntzinger, R. C. Craven, and I. A. Holder. 1982. Loss of virulence associated with absence of flagellum in an isogenic mutant of Pseudomonas aeruginosa in the burned-mouse model. Infect. Immun. 38:1296-1298.

88. Motaleb, M. A., L. Corum, J. L. Bono, A. F. Elias, P. Rosa, D. S. Samuels, and N. W. Charon. 2000. Borrelia burgdorferi periplasmic flagella have both skeletal and motility functions. Proc. Natl. Acad. Sci. USA 97:10899-904.

89. Motaleb, M. A., M. S. Sal, and N. W. Charon. 2004. The decrease in FlaA observed in a flaB mutant of Borrelia burgdorferi occurs posttranscriptionally. J. Bacteriol. 186:3703-3711.

90. Muir, R. E. and J. W. Gober. 2001. Regulation of late flagellar gene transcription and cell division by flagellum assembly in Caulobacter crescentus. Mol. Microbiol. 41:117-130.

91. Mullin, D. A., N. Ohta, A. H. Mullin, and A. Newton. 2001. Organization, expression, and function of Caulobacter crescentus genes needed for assembly and function of the flagellar hook. Mol. Genet. Genomics 265:445-454.

92. Nadelman, R. B. and G. P. Wormser. 1998. Lyme borreliosis. Lancet 352:557-65.

93. Nambu, T. and K. Kutsukake. 2000. The Salmonella FlgA protein, a putativeve periplasmic chaperone essential for flagellar $\mathrm{P}$ ring formation. Microbiology 146:1171-1178. 
94. Norris, S. J. 1993. Polypeptides of Treponema pallidum: progress toward understanding their structural, functional, and immunologic roles. Treponema Pallidum Polypeptide Research Group. Microbiol. Rev. 57:750-79.

95. Norris, S. J., N. W. Charon, R. G. Cook, M. D. Fuentes, and R. J. Limberger. 1988. Antigenic relatedness and $\mathrm{N}$-terminal sequence homology define two classes of periplasmic flagellar proteins of Treponema pallidum subsp. pallidum and Treponema phagedenis. J. Bacteriol. 170:4072-4082.

96. Norris, S. J., J. K. Howell, S. A. Garza, M. S. Ferdows, and A. G. Barbour. 1995. High- and low-infectivity phenotypes of clonal populations of in vitro-cultured Borrelia burgdorferi. Infect. Immun. 63:2206-2212.

97. Novotna, M. and O. Skardova. 2002. Brachyspira hyodysenteriae: detection, identification, and antibiotic susceptibility. Vet. Med.- Czech 47:104-109.

98. Novotna, M. and O. Skardova. 2004. Brachyspira hyodysenteriae: detection, identification, and antibiotic susceptibility. Vet. Med.- Czech 47:104-109.

99. Ochi, H., H. Ohtsuka, S. Yokota, I. Uezumi, M. Terashima, K. Irie, and H. Noguchi. 1991. Inhibitory activity on bacterial motility and in vivo protective activity of human monoclonal antibodies against flagella of Pseudomonas aeruginosa. Infect. Immun. 59:550-554.

100. Ochiai, S., Y. Adachi, and K. Mori. 1997. Unification of the genera Serpulina and Brachyspira, and proposals of Brachyspira hyodysenteriae Comb. Nov., Brachyspira innocens Comb. Nov. and Brachyspira pilosicoli Comb. Nov. Microbiol. Immunol. 41:445-452.

101. Ohnishi, K., K. Kutsukake, H. Suzuki, and T. lino. 1990. Gene fliA encodes an alternative sigma factor specific for flagellar operons in Salmonella typhimurium. Mol. Gen. Genet. 221:139-147.

102. Ohnishi, K., Y. Ohto, S. Aizawa, R. M. Macnab, and T. lino. 1994. FlgD is a scaffolding protein needed for flagellar hook assembly in Salmonella typhimurium. J. Bacteriol. 176:2272-2281.

103. Pal, U., A. M. de Silva, R. R. Montgomery, D. Fish, J. Anguita, J. F. Anderson, Y. Lobet, and E. Fikrig. 2000. Attachment of Borrelia burgdorferi within Ixodes scapularis mediated by outer surface protein $A$. J. Clin. Invest. 106:561-9. 
104. Pallesen, L. and P. Hindersson. 1989. Cloning and sequencing of a Treponema pallidum gene encoding a 31.3-kilodalton endoflagellar subunit (FlaB2). Infect. Immun. 57:2166-2172.

105. Paster, B. J. and E. Canale-Parola. 1980. Involvement of periplasmic fibrils in motility of spirochetes. J. Bacteriol. 141:359-364.

106. Paster, B. J. and F. E. Dewhirst. 2000. Phylogenetic foundation of spirochetes. J. Mol. Microbiol. Biotechnol. 2:341-4.

107. Pavlovskis, O. R., D. M. Rollins, R. L. Haberberger, Jr., A. E. Green, L. Habash, S. Strocko, and R. I. Walker. 1991. Significance of flagella in colonization resistance of rabbits immunized with Campylobacter spp. Infect. Immun. 59:2259-2264.

108. Pleier, E. and R. Schmitt. 1989. Identification and sequence analysis of two related flagellin genes in Rhizobium meliloti. J. Bacteriol. 171:14671475.

109. Pleier, E. and R. Schmitt. 1991. Expression of two Rhizobium meliloti flagellin genes and their contribution to the complex filament structure. J. Bacteriol. 173:2077-2085.

110. Prouty, M. G., N. E. Correa, and K. E. Klose. 2001. The novel sigma54and sigma28-dependent flagellar gene transcription hierarchy of Vibrio cholerae. Mol. Microbiol. 39:1595-609.

111. Prpic, J. K., F. Trewartha, and S. R. Graves. 1981. Enhanced retention of motility and virulence of Treponema pallidum (Nichols strain) in vitro by the addition of gelatin to anaerobic medium. Sex. Transm. Dis. 8:1-4.

112. Purser, J. E., M. B. Lawrenz, M. J. Caimano, J. K. Howell, J. D. Radolf, and S. J. Norris. 2003. A plasmid-encoded nicotinamidase (PncA) is essential for infectivity of Borrelia burgdorferi in a mammalian host. Mol. Microbiol. 48:753-764.

113. Richardson, K. 1991. Roles of motility and flagellar structure in pathogenicity of Vibrio cholerae: analysis of motility mutants in three animal models. Infect. Immun. 59:2727-2736.

114. Rohde, J., A. Rothkamp, and G. F. Gerlach. 2002. Differentiation of porcine Brachyspira species by a novel nox PCR-based restriction fragment length polymorphism analysis. J. Clin. Microbiol. 40:2598-2600.

115. Rosa, P., D. S. Samuels, D. Hogan, B. Stevenson, S. Casjens, and K. Tilly. 1996. Directed insertion of a selectable marker into a circular plasmid of Borrelia burgdorferi. J. Bacteriol. 178:5946-53. 
116. Rosey, E. L., M. J. Kennedy, D. K. Petrella, R. G. Ulrich, and R. J. Yancey, Jr. 1995. Inactivation of Serpulina hyodysenteriae flaA1 and flaB1 periplasmic flagellar genes by electroporation-mediated allelic exchange. J. Bacteriol. 177:5959-5970.

117. Rosey, E. L., M. J. Kennedy, and R. J. Yancey, Jr. 1996. Dual flaA1 flaB1 mutant of Serpulina hyodysenteriae expressing periplasmic flagella is severely attenuated in a murine model of swine dysentery. Infect. Immun. 64:4154-62.

118. Ruby, J. D. and N. W. Charon. 1998. Effect of temperature and viscosity on the motility of the spirochete Treponema denticola. FEMS Microbiol. Lett. 169:251-4.

119. Ruby, J. D., H. Li, H. Kuramitsu, S. J. Norris, S. F. Goldstein, K. F. Buttle, and N. W. Charon. 1997. Relationship of Treponema denticola periplasmic flagella to irregular cell morphology. J. Bacteriol. 179:1628-35.

120. Sadziene, A., D. D. Thomas, V. G. Bundoc, S. C. Holt, and A. G. Barbour. 1991. A flagella-less mutant of Borrelia burgdorferi. Structural, molecular, and in vitro functional characterization. J. Clin. Invest. 88:8292.

121. Saito, T., T. Ueno, T. Kubori, S. Yamaguchi, T. lino, and S. I. Aizawa. 1998. Flagellar filament elongation can be impaired by mutations in the hook protein FlgE of Salmonella typhimurium: a possible role of the hook as a passage for the anti-sigma factor FlgM. Mol. Microbiol. 27:1129-1139.

122. Samuels, D. S. 1995. Electrotransformation of the Spirochete Borrelia burgdorferi, p. 253. In J. A. Nickoloff (ed.), Methods of Molecular Biology. Humana Press, Totowa, New Jersey.

123. Sartakova, M., E. Dobrikova, and F. C. Cabello. 2000. Development of an extrachromosomal cloning vector system for use in Borrelia burgdorferi. Proc. Natl. Acad. Sci. USA 97:4850-5.

124. Sartakova, M. L., E. Y. Dobrikova, M. A. Motaleb, H. P. Godfrey, N. W. Charon, and F. C. Cabello. 2001. Complementation of a nonmotile flaB mutant of Borrelia burgdorferi by chromosomal Integration of a plasmid containing a wild-type flaB allele. J. Bacteriol. 183:6558-64.

125. Schwan, T. G. and W. Burgdorfer. 1987. Antigenic changes of Borrelia burgdorferi as a result of in vitro cultivation. J. Infect. Dis. 156:852-3.

126. Schwan, T. G., W. Burgdorfer, and C. F. Garon. 1988. Changes in infectivity and plasmid profile of the Lyme disease spirochete, Borrelia burgdorferi, as a result of in vitro cultivation. Infect. Immun. 56:1831-1836. 
127. Schwan, T. G. and J. Piesman. 2000. Temporal changes in outer surface proteins $\mathrm{A}$ and $\mathrm{C}$ of the lyme disease-associated spirochete, Borrelia burgdorferi, during the chain of infection in ticks and mice. J. Clin. Microbiol. 38:382-388.

128. Singh, S. K. and H. J. Girschick. 2004. Lyme borreliosis: from infection to autoimmunity. Clin. Microbiol. Infect. 10:598-614.

129. Skirrow, S. 2004. Swine Dysentery. Farmnote (17) Department of Agriculature, Western Australia.

130. Stanton, T. B. 1992. Proposal to change the genus designation Serpula to Serpulina gen. nov. containing the species Serpulina hyodysenteriae comb. nov. and Serpulina innocens comb. nov. Int. J. Syst. Bacteriol. 42:189-190.

131. Stanton, T. B., E. G. Matson, and S. B. Humphrey. 2001. Brachyspira (Serpulina) hyodysenteriae gyrB mutants and interstrain transfer of coumermycin A(1) resistance. Appl. Environ. Microbiol. 67:2037-2043.

132. Stanton, T. B., M. G. Thompson, S. B. Humphrey, and R. L. Zuerner. 2003. Detection of bacteriophage VSH-1 svp38 gene in Brachyspira spirochetes. FEMS Microbiol.Lett. 224:225-229.

133. Stewart, P. E., R. Thalken, J. L. Bono, and P. Rosa. 2001. Isolation of a circular plasmid region sufficient for autonomous replication and transformation of infectious Borrelia burgdorferi. Mol. Microbiol. 39:714-21.

134. ter Huurne, A. A., M. van Houten, S. Muir, J. G. Kusters, B. A. van der Zeijst, and W. Gaastra. 1992. Inactivation of a Serpula (Treponema) hyodysenteriae hemolysin gene by homologous recombination: importance of this hemolysin in pathogenesis in mice. FEMS Microbiol. Lett. 71:109-113.

135. Trott, D. J., C. R. Huxtable, and D. J. Hampson. 1996. Experimental infection of newly weaned pigs with human and porcine strains of Serpulina pilosicoli. Infect. Immun. 64:4648-4654.

136. Trott, D. J., T. B. Stanton, N. S. Jensen, G. E. Duhamel, J. L. Johnson, and D. J. Hampson. 1996. Serpulina pilosicoli sp. nov., the agent of porcine intestinal spirochetosis. Int. J. Syst. Bacteriol. 46:206-215.

137. Turner, L., W. S. Ryu, and H. C. Berg. 2000. Real-time imaging of fluorescent flagellar filaments. J. Bacteriol. 182:2793-801.

138. Vonderviszt, F., P. Zavodszky, M. Ishimura, H. Uedaira, and K. Namba. 1995. Structural organization and assembly of flagellar hook protein from Salmonella typhimurium. J. Mol. Biol. 251:520-532. 
139. Weissborn, A., H. M. Steinmann, and L. Shapiro. 1982.

Characterization of the proteins of the Caulobacter crescentus flagellar filament. Peptide analysis and filament organization. J. Biol. Chem.

257:2066-2074.

140. Wu, J., A. K. Benson, and A. Newton. 1995. Global regulation of a sigma 54-dependent flagellar gene family in Caulobacter crescentus by the transcriptional activator FlbD. J. Bacteriol. 177:3241-3250.

141. Wu, J. and A. Newton. 1997. Regulation of the Caulobacter flagellar gene hierarchy; not just for motility. Mol. Microbiol. 24:233-239.

142. Xu, Y., C. Kodner, L. Coleman, and R. C. Johnson. 1996. Correlation of plasmids with infectivity of Borrelia burgdorferi sensu stricto type strain B31. Infect. Immun. 64:3870-3876.

143. Zuerner, R. L. and T. B. Stanton. 1994. Physical and genetic map of the Serpulina hyodysenteriae B78T chromosome. J. Bacteriol. 176:10871092. 
Chapter 2. The decrease in FlaA observed in a flaB mutant of Borrelia burgdorferi occurs posttranscriptionally

This work was published as:

Md. A. Motaleb, Melanie Sal, and Nyles W. Charon. 2004. The decrease in FlaA observed in a flaB mutant of Borrelia burgdorferi occurs posttranscriptionally. J. Bact. 186(12):3703-3711. 


\section{Abstract}

The Lyme disease bacterium Borrelia burgdorferi is a motile spirochete with a flat-wave morphology. The periplasmic flagella, which are situated between the outer membrane sheath and cell cylinder, are essential for both the cell's wavy shape and motility. Here we focus on the structure and regulation of its periplasmic flagella. Previous studies have suggested that the periplasmic flagella consist of a polymer of the major filament protein FlaB and a minor protein FlaA. We used immunoprecipitation methodology to present further evidence that FlaA is indeed a flagellar protein. In addition, in contrast to FlaA of the spirochete Brachyspira hyodysenteriae, B. burgdorferi FlaA did not impact the overall helical shape of the periplasmic flagella. We have previously shown that $B$. burgdorferi lacks the sigma factor dependent cascade control of motility gene transcription found in other bacteria. To begin to understand motility gene regulation in $B$. burgdorferi, we examined the effects of an insertion mutation in flaB on the amounts of proteins encoded by motility genes. Of several motility gene encoded proteins examined, only the amount of FlaA was decreased in the flaB mutant; it was $13 \%$ compared to the wild-type. Real time reverse transcriptase polymerase chain reaction analysis indicated that this inhibition was not the result of a decrease in flaA mRNA. In addition, protein stability analysis suggested that FlaA was turned over in the flaB mutant. Our results indicate that the lack of FlaB negatively influences the amount of FlaA found in the cell, and that this effect is at the level of either translational control or protein turnover. 


\section{Introduction}

Borrelia burgdorferi is a motile spirochete that is the causative agent of Lyme disease. The organelles for motility, the periplasmic flagella (PFs), reside between the outer membrane sheath and protoplasmic cell cylinder. Approximately 7-11 PFs are subterminally attached at each end of the cell, and these filaments overlap in the center of the cell $(4,28,32)$. Motility is likely to be an important virulence factor for these spirochetes, as $B$. burgdorferi penetrates into tissues where other organisms fail to invade $(11,35)$. Moreover, motility has been postulated to be essential for the life cycle of these spirochetes in both the tick and the mammalian hosts $(45,55)$.

We are beginning to understand the dynamics of $B$. burgdorferi motility (see 11, 42, 45 for recent reviews). Recent results indicate that the PFs have both skeletal and motility functions $(50,61)$. Mutants that are targeted in flaB, which encodes the major PF protein, are non-motile and are rod-shaped instead of being a flat-wave as are wild-type cells $(50,61)$. During translational motility, backward moving waves are generated that are responsible for cell displacement (29). These waves result from the rotation of the relatively rigid left-handed helically shaped PFs juxtaposed close to and interacting with the flexible protoplasmic cell cylinder: The PFs act as a worm gear generating waves from the anterior to the posterior end of the cell $(11,28,29,42)$. The bundles of PFs rotate asymmetrically, with the anterior bundle rotating counter-clockwise (CCW) and the posterior one clockwise $(\mathrm{CW})$ as viewed along the length of the PFs from their distal ends towards their insertion points on the cell $(11,29,42)$. 
The PFs of $B$. burgdorferi have been analyzed in some detail. These organelles have been shown to be left-handed with a defined helix pitch and diameter (12). Their major constituent is a polymer of FlaB, which is similar in sequence at the $\mathrm{N}$ - and $\mathrm{C}$-terminal domains to PFs of other spirochete species as well as flagellin of other bacteria $(53,63)$. Based on purification of PFs, FlaB was considered for several years to be the only filament protein. However, DNA sequence analysis indicated the presence of a flaA homolog $(18,21)$. In other spirochete species, FlaA forms a sheath around the FlaB core. In addition, in Brachyspira hyodysenteriae, FlaA markedly affects the shape of the PFs with respect to helix pitch and helix diameter $(9,39,41,42,53)$. We found that flaA was expressed in B. burgdorferi, and by employing a more gentle method of isolation, FlaA co-purified with the PFs (23). However, in contrast to other spirochete species $(9,39,41,42,53,59)$, considerably less FlaA relative to FlaB was present in these purified PFs. Using immunoprecipitation, we provide further evidence that FlaA is associated with FlaB. In addition, we find that in contrast to other spirochetes, there is considerably less FlaA relative to FlaB not only in purified PFs, but also within the whole cell. Finally, we show that FlaA does not affect PF shape of $B$. burgdorferi as it does for $B$. hyodysenteriae.

Bacterial flagella are complex organelles that often comprise a disproportionately large amount of protein in a motile cell. As a result, bacteria have evolved elegant mechanisms of flagellar assembly and control. Specifically, 
motile bacteria possess a cascade system of transcriptional control $(3,15,48,1$, 2, 49). For example, in Escherichia coli and Salmonella enterica serovar Typhimurium, the cell senses specific environmental signals to commence expression of class 1 genes $f / h C$ and $f / h D$. FlhC and FlhD then promote the initiation of transcription of class 2 motility genes $(2,15)$. These genes include, among others, those that encode the proteins that comprise the basal body apparatus. Two of the class 2 genes, fliA and flgM, play critical roles in regulating class 3 gene transcription. Specifically, fliA encodes sigma ${ }^{28}$ that promotes transcription of class 3 genes including the flagellar filament gene fliC. flgM encodes an anti-sigma ${ }^{28}$ factor, $\operatorname{FlgM}(2,15)$, which is excreted into the medium when the flagellar hook-basal body is complete. FlgM insures that the class 3 genes are not transcribed until the initial flagellar structure is intact, thus providing the cell with a mechanism to sense the state of flagellar synthesis and adjust its transcription accordingly $(2,15)$.

The regulation of motility gene expression in $B$. burgdorferi is unique, as it lacks the cascade control of flagellar gene transcription found in other bacteria. Several studies indicate that gene transcription of the numerous motility operons is initiated by the housekeeping transcription factor sigma ${ }^{70}(11,18,21,22,25$, $26,42)$. Furthermore, no fliA homolog has been identified in its genome $(18,42)$. To begin to understand how the motility genes are regulated in B. burgdorferi, we are examining the effects of specific PF mutations on the synthesis of several 
motility and chemotaxis gene products. In this communication, we report our results in a previously described insertion mutant in flaB $(50,61)$. 


\section{Materials and Methods}

Bacterial strains and growth conditions. High-passage B. burgdorferi sensu stricto strain $\mathrm{B} 31 \mathrm{~A}$, the flaB mutant $\mathrm{MC}-1$, and the complemented flaB mutant MS17 have been previously described $(8,50,61)$. Briefly, MC-1 has a flgB promoter-kanamycin cassette inserted into flaB of $B$. burgdorferi. This mutant is non-motile and fails to synthesize PFs (50). MS17 is a derivative of MC-1 containing a wild-type copy of flaB in the plasmid pED3. pED3 complements the flaB mutation in cis, i.e. only when the plasmid is integrated into the chromosome by a Campbell type recombination event (61). Cells were grown in BSK-II broth or on plates at $32^{\circ} \mathrm{C}-34^{\circ} \mathrm{C}$ as previously described (50). S. enterica serotype Typhimurium strain SJW1103 is wild-type for chemotaxis and flagellation, and strain SJW1368 $\Delta(c h e W-f l h D)$ is a master operon mutant which is aflagellate (19). These strains were the kind gift of the late R. Macnab, Yale University.

Gel electrophoresis and Western blot analysis. Sodium dodecyl sulphatepolyacrylamide gel electrophoresis (SDS-PAGE) and Western blotting with an enhanced chemi-luminescent detection method (ECL, Amersham Pharmacia) were carried out as previously reported (23). Equal amounts (except as noted) of cellular protein in lysates, as determined by a Bio-Rad protein assay kit, were applied to each lane. Several monoclonal and polyclonal antibodies were kindly provided by other investigators and include the following: monoclonal anti-FlaA (B. Johnson, Centers for Disease Control, Atlanta, GA), monoclonal anti-OspA and DnaK (J. Benach, State University of New York, Stony Brook, NY), 
monoclonal anti-FlaB (H9724, A. Barbour, University of California, Irvine), rabbit anti-MotB (J. Carroll, Rocky Mountain Laboratories, Hamiliton, MT), rabbit polyclonal anti-FlaA of T. pallidum (S. Norris, University of Texas, Houston), rabbit anti-CheA of E. coli (P. Matsumura, University of Illinois, Chicago, IL), rabbit anti-FliM of E. coli (D. Blair, University of Utah, Salt Lake City, UT). Rabbit anti-CheY2 and anti-FlgE will be described elsewhere (M. Motaleb, M. Sal and N. Charon, manuscript in preparation). Specific monoclonal or polyclonal reactivity to $B$. burgdorferi DnaK, FlaA, FlaB, Flil, MotB, CheA1, CheA2, CheY3 has been previously reported $(16,5,10,24,40,50,54)$. Insertion mutants in flaA, flaB, flgE, motB, cheA1, cheA2, cheY2, and cheY3 verified antibody specificity by Western blotting (M. Motaleb, M. Sal, N. Charon, unpublished; $(40,50))$. AntiFliM reactivity was verified using S. typhimurium strains SJW1103 and SJW1368.

Quantitative immunoblot assays. To quantify FlaA and FlaB in cell lysates, PFs were purified using the Triton X-100 method as previously described (23). PF proteins were separated on SDS-PAGE gels, and the bands corresponding to FlaA and FlaB were cut from the gels which were then electroeluted using ExElutor (Labnet International Inc., Woodbridge, NJ). FlaA and FlaB were concentrated by centrifugation in Amicon Ultra concentrators (Millipore, Bedford, MA). Protein concentrations were determined using the Bio-Rad protein assay kit, as well as by SDS-PAGE gels stained with Coomassie Brilliant Blue R-250 with bovine serum albumin (BSA) as standard. Different amounts of purified FlaA, FlaB, and cell lysates were probed with monoclonal anti-FlaA and anti-FlaB 
using Western blotting. Signals were quantified by FluorChem spot densitometry (Alpha Innotech, San Leandro, CA).

Immunoprecipitation and PF morphology. Immunoprecipitation was carried out as previously described (43). Approximately $5 \times 10^{9} \mathrm{~B}$. burgdorferi cells were washed twice with $150 \mathrm{mM}$ phosphate buffered saline (PBS, $\mathrm{pH} 7.5)-5 \mathrm{mM} \mathrm{MgCl}_{2}$, and were lysed by incubation at $37^{\circ} \mathrm{C}$ for $1 \mathrm{hr}$ in TSEA buffer $(50 \mathrm{mM}$ Tris- $\mathrm{HCl}$, $150 \mathrm{mM} \mathrm{NaCl}, 5 \mathrm{mM}$ EDTA, 0.05\% Na-Azide, pH 7.5) containing 1\% Nonidet P40 and $50 \mu \mathrm{g} / \mathrm{ml}$ of phenylmethylsulfonyl fluoride. The lysate was centrifuged $\left(1,600 \times g\right.$ for $\left.30 \mathrm{~min}, 25^{\circ} \mathrm{C}\right)$, and the pellet was resuspended in PBS and sonicated for $1 \mathrm{~min}$ intermittently for 20 minutes in ice followed by centrifugation $\left(14,800 \times \mathrm{g}\right.$ for $\left.30 \mathrm{~min}, 25^{\circ} \mathrm{C}\right)$. Approximately $200 \mu \mathrm{l}$ of the supernatant fluid were incubated with $15 \mu \mathrm{l}$ of the monoclonal anti-FlaB for $1 \mathrm{hr}$ at $25^{\circ} \mathrm{C}$ in the presence of $1 \%$ BSA. Controls consisted of anti-FlaB incubated with no cell sonicate, and the cell sonicate incubated with no antibody. After incubation, approximately 50 $\mu$ l of Protein A (Pansorbin; Staphylococcus aureus Cowan 1, Calbiochem Behring, La Jolla,CA) were added to each sample, and further incubated for $1 \mathrm{hr}$ at $25^{\circ} \mathrm{C}$. The immunoprecipitates and controls were centrifuged at $1,600 \times \mathrm{g}$ at $25^{\circ} \mathrm{C}$ and washed three times with $1 \mathrm{ml}$ of TSEA buffer containing $0.05 \%$ Tween20. The final pellets were suspended in $60 \mu \mathrm{l}$ of electrophoresis sample buffer, boiled for 5 minutes, and briefly centrifuged as before. For Western blotting, $10 \mu \mathrm{l}$ of the supernatant fluids were applied to each lane of a SDS-PAGE gel. The handedness, helical pitch, and helical diameter of the purified PFs were 
determined as previously described using dark-field microscopy $(12,23,41)$. PFs containing both FlaA and FlaB were purified as previously described using the Triton X-100 method (23). PFs containing FlaB and no FlaA were purified with Sarkosyl as previously reported $(12,23)$.

\section{Real-time quantitative reverse transcriptase-PCR (RT-PCR) and RNase} protection assays. Total cellular RNA was extracted using the RNeasy kit from Qiagen and then digested with Turbo DNase I (Ambion) at $37^{\circ} \mathrm{C}$ for 2 hours. Equal amounts of total RNA (approximately $0.3 \mu \mathrm{g}$ ) were used to generate cDNA according to the manufacturers protocol (Promega). To ensure that the RNA preparations were free from genomic DNA, reverse transcription was performed with or without reverse transcriptase. Quantitative-PCR was carried out in duplicate on cDNA with the SYBR Green master mix (Qiagen) using a LightCycler (Roche Biochemicals). A standard curve was generated using 10-fold serial dilutions ( $1.0 \mathrm{pg}$ to $0.1 \mathrm{fg}$ ) from the flaA RT-PCR product. $16 \mathrm{~S}$ rRNA was used as an internal control. PCR primers for flaA (BB0668, Genebank accession no. U62900) were the following: forward primer, 5'CACTAGTTCTTTTTTGCTCAA-3'; reverse primer, 5'ACTGCAGTACCTTTTTTGACTC-3'. For 16S rRNA (Genebank accession no. L40596), the primers were the following: forward primer, 5'GTGGCGAACGGGTGAGTAAC-3'; reverse primer, 5'CCGTCAGCTTTCGCCATTGC-3'. Results are expressed as the threshold cycle $\left(C_{t}\right)$, at which the measured fluorescence increased above background (57). 
RNase protection assays were carried out according to standard procedures using a Hybspeed RPA kit from Ambion (23). Briefly, primers to the B. burgdorferi flaA and $16 \mathrm{~S}$ rRNA were used to amplify specific regions by PCR. The amplified regions (305-bp flaA and 296-bp 16S rRNA) were cloned into pGEM-T Easy vector (Promega), and the resultant plasmids were linearized with Spel (flaA) or Ncol (16S rRNA). Antisense riboprobe was synthesized with [ $\alpha-$ 32P]UTP (Amersham Pharmacia) using a transcription kit (Ambion) and purified using a 5\% polyacrylamide-8M urea gel. Hybridization and digestion were done as described previously (23). Transcript levels were quantified using a Molecular Dynamics Phospholmager.

Protein turnover. Techniques previously employed in other bacteria (34) were used to determine the stability of FlaA in growing cells. Wild-type and MC-1 strains were grown in $\mathrm{BSK}-\mathrm{Il}$ medium at $33^{\circ} \mathrm{C}$ until cells reached a density of $5 \times 10^{8}$ cells per $\mathrm{ml}$. Two $\mathrm{ml}$ of cells were added to $40 \mathrm{ml}$ of BSK-II medium containing spectinomycin (final concentration $100 \mu \mathrm{g} / \mathrm{ml}$ ), and incubated at $33^{\circ} \mathrm{C}$. Samples were removed and processed for Western blotting at indicated time points. 


\section{Results}

Association of FlaA with FlaB. We previously found that FlaA and FlaB copurified when using the Triton $\mathrm{X}-100$ procedure for PF isolation (23). To confirm that these two proteins were actually associated with one another, FlaB was immunoprecipatated from cell lysates of $B$. burgdorferi, and the resulting immunoprecipitate was probed with anti-FlaB (Figure 1, left) and anti-FlaA (Figure 1, right) in Western blotting. We found that FlaA co-precipitated with FlaB (Figure 1, right), while no co-precipitation occurred with another abundant $B$. burgdorferi protein, OspA (not shown). These results suggest that there is a specific association of FlaA with FlaB in situ.

FlaA is a minor PF protein. SDS-PAGE of purified PFs was previously used to estimate the relative amounts of FlaA and FlaB in B. burgdorferi. In these studies, FlaA was found to be a minor component (23). These results are in contrast to other spirochete species whereby the amount of FlaA is approximately equal to that of $\operatorname{FlaB}(9,41,53,54,59)$. One concern is that perhaps much of the B. burgdorferi FlaA was lost during the purification of PFs. Accordingly, we determined the amount of both FlaA and FlaB in total cell lysates using quantitative immunoblotting. Western blot results indicated that FlaA contributed to less than 0.5 percent to the total protein of the cell (Figure 2a). In contrast, FlaB was found to contribute $10-14$ percent of the total cell protein (Figure 2b). 


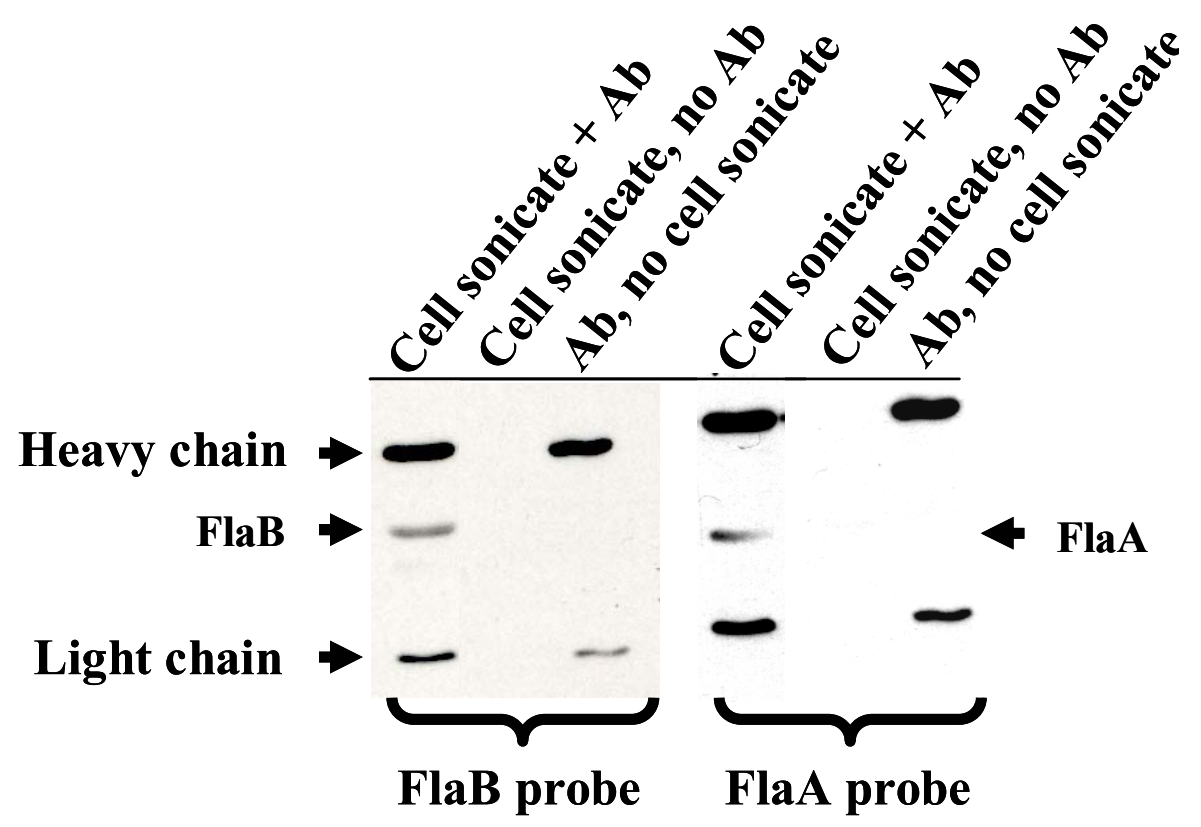

Figure 1. Immunoprecipitation of FlaA with monoclonal H9724 anti-FlaB

Wild-type cell sonicates were immunoprecipitated with FlaB antibody (Ab) and then probed with monoclonal $\mathrm{Ab}$ to FlaB (left panel) and FlaA (right panel) by Western blotting. The upper and lower bands of heavy and light chains of the primary $\mathrm{Ab}$ are identified. In controls without FlaB $\mathrm{Ab}$, neither FlaB nor FlaA were precipitated (middle lanes). 
This relatively large amount of FlaB is in agreement with other multiflagellated bacteria. In S. enterica serovar Typhimurium, which has approximately 8 flagella per cell, the filament protein FliC contributes 8 per cent of the total cell protein (51). B. hyodysenteriae has approximately 10-13 PFs per bundle, and these PFs have been estimated to contribute 5 - 10 per cent of total protein (39). Our results are consistent with the PFs being a major constituent of $B$. burgdorferi, and that these organisms accumulate considerably less FlaA than FlaB.

Influence of FlaA on PF structure. FlaA has been shown to affect periplasmic flagellar structure of $B$. hyodysenteriae. Specifically, PFs from mutants that lack FlaA have a markedly different helix pitch and helix diameter than that of the wild-type (41). B. burgdorferi PFs were previously shown to be left-handed, and have a helix pitch of $1.48 \pm 0.03 \mu \mathrm{m}$ and helix diameter of $0.28 \pm 0.03 \mu \mathrm{m}$. These measurements were made with PFs isolated using Sarkosyl; such PFs lack FlaA (12). To examine whether FlaA influenced PF shape of B. burgdorferi, we isolated PFs containing both FlaA and FlaB using the more gentle technique that has sarkosyl replaced with triton X-100 (23). We found that PFs with both FlaA and FlaB were identical in shape to those isolated without FlaA (left handed, helix pitch $1.5 \pm 0.028 \mu \mathrm{m}$, helix diameter $0.33 \pm 0.027 \mu \mathrm{m}$, Figure $2.2 \mathrm{c})$. The results indicate that, in contrast to $B$. hyodysenteriae, FlaA does not influence the helical shape of the PFs. 
(a)

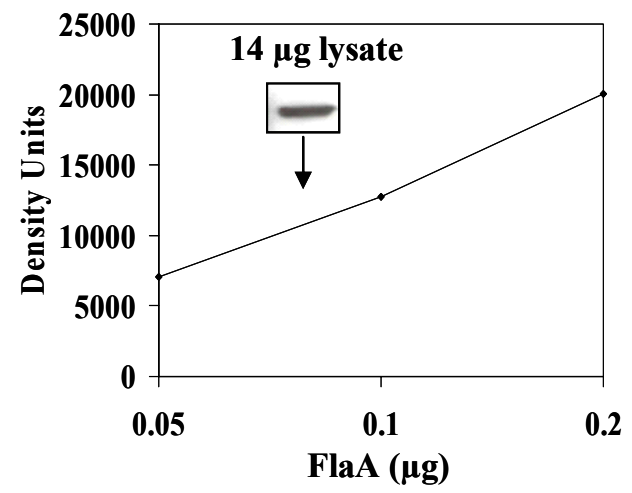

(b)

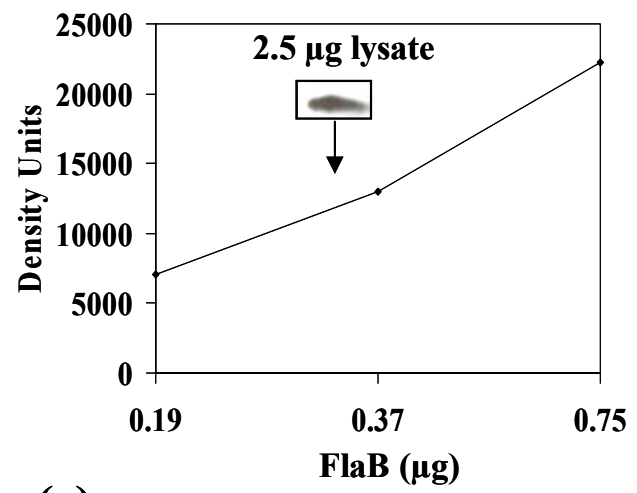

(c)

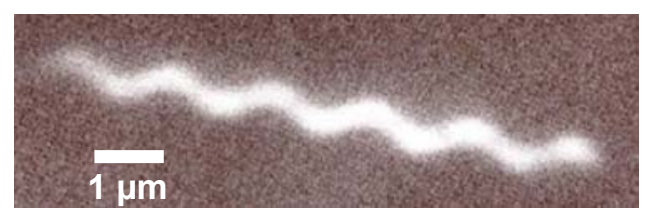

Figure 2. Quantitative Western blot analysis of PF protein levels in wild type $B$. burgdorferi.

Different amounts of gel purified $\mathrm{FlaA}, \mathrm{FlaB}$, and cell lysates were separated and probed with monoclonal anti-FlaA (a), and anti-FlaB (b) antibodies. The amounts of FlaA and FlaB in the cell lysates were calculated from the standard curves obtained from purified FlaA (a) and FlaB (b), respectively. Inserts illustrate Western blot reactivity at a specific amount of protein lysate. 


\section{Influence of flaB::kan on the synthesis of select gene products in motility}

operons. Previous results indicated that $B$. burgdorferi lacks the typical cascade control of flagellar gene regulation seen in other bacteria $(11,18,21,22,25,26$, 42). To begin to understand how motility genes are regulated in $B$. burgdorferi, we examined the effects of a flaB::kan mutation (mutant MC-1) on the synthesis of other flagella and chemotaxis proteins. The flagellar filament gene flaB maps far to the left on the linear B. burgdorferi chromosome (18) and is transcribed as a monocistronic mRNA $(20,60)$. We first examined protein synthesis with respect to select genes in the large $21 \mathrm{~kb} f l g B$ operon that encodes most of the hook, basal body, and motility genes. This operon maps approximately $180 \mathrm{~kb}$ away from flaB (18). Those genes chosen for analysis were flil, flgE, fliM, and motB; each maps several open-reading frames away from one another. Flil in other bacteria is associated with both basal body protein and flagellin export. FlgE is the flagellar hook structural protein which serves as a universal joint between the flagellar basal body and the filament (6). FliM is part of the rotary apparatus of the motor, and MotB is the stator involved in energy transduction to the motor (6). Insertion mutations in $f l g E$ and motB led to the expected non-motile phenotypes (M. Motaleb, M. Sal, N. Charon, unpublished; 61), indicating that these genes function in motility as predicted from their sequence. Western blot analysis indicated that although the synthesis of FlaB was inhibited in MC-1 as reported previously (50), no decrease in the amount of protein is evident with respect to FlgE, Flil, FliM, and MotB (Figure 3). These results suggest that flaB::kan did not 
alter the synthesis of the encoded proteins for the select motility genes assayed in this operon.

We examined the effect of the flaB mutation on a putative chemotaxis operon, which is approximately $400 \mathrm{~kb}$ away from flaB. This operon consists of cheW2, orf566, cheA1, cheB2, orf569, cheY2. Preliminary results indicate that mutants in cheW2, orf569, and cheY2 negatively impact chemotaxis (C. Li, M. Motaleb, and N. Charon, unpublished). However, although Western blot analysis indicated that CheA1 was produced, it was found not to be essential for chemotaxis (40). We tested whether flaB::kan affected the expression of CheA1 and CheY2. Western blot analysis indicated that the levels of CheA1 and CheY2 in the MC-1 mutant were equal to that of the wild-type (Figure 3). These results indicate that flaB::kan did not influence the amount of CheA1 and CheY2. 


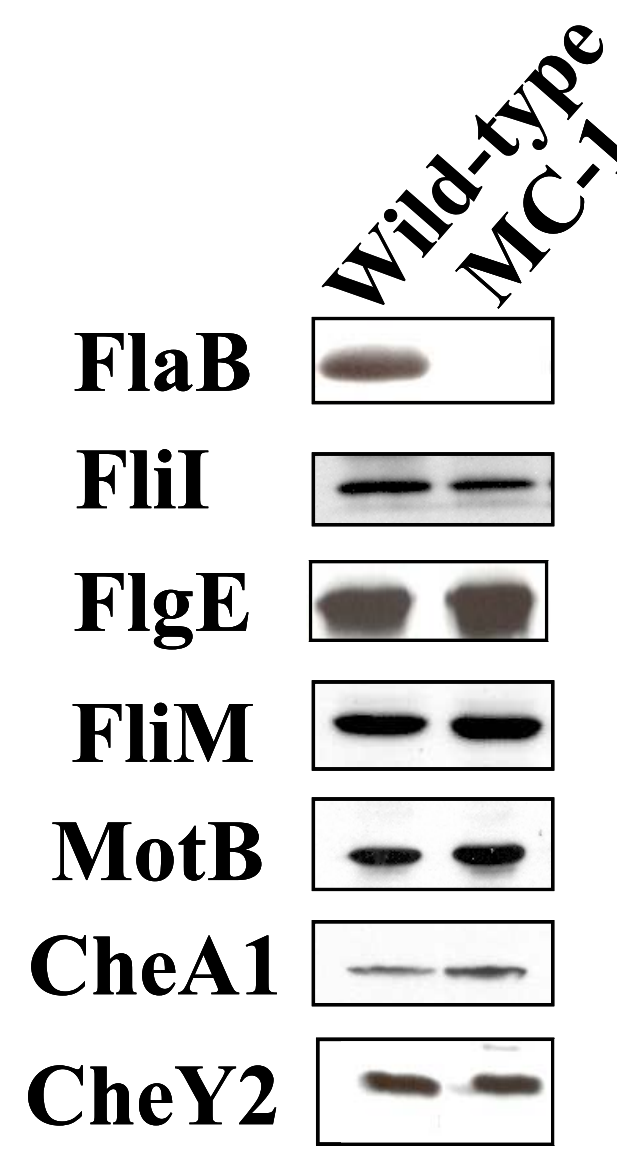

Figure 3. Effect of flaB::kan on other motility and chemotaxis proteins.

Equal amounts $(10 \mu \mathrm{g})$ of cell lysates from wild-type and the flaB::kan mutant strain MC-1 were probed by Western blot with antibodies specific for the indicated proteins. DnaK was used as an internal control as shown in Fig. $4 \mathrm{~b}$. The apparent sizes of the proteins are the following (kD): FlaB, 41; Flil, 48; FlgE, 40; FliM, 37; MotB, 24; CheA1, 80; CheY2, 12. 
Protein synthesis of select genes in the flaA operon. Another relatively large operon consists of flaA-cheA2-cheW3-cheX-cheY3 $(21,23,25)$. It maps approximately $550 \mathrm{~kb}$ away from flaB. Targeted mutations in cheA2, cheX and cheY3 markedly affect chemotaxis in B. burgdorferi (M.A. Motaleb and N.W. Charon, unpublished; 40). We tested the effect of the flaB::kan mutation on the synthesis of FlaA, CheA2, and CheY3. Western blot analysis indicated that both CheA2 and CheY3 in MC-1 were synthesized at levels equivalent to the wild-type (Figure 4a). However, Western blots probed with either a heterologous polyclonal FlaA antiserum (Figure 4a), or a monoclonal antibody directed to $B$. burgdorferi FlaA (Figure 4b), indicated that the level of FlaA in MC-1 was markedly decreased.

Using the monoclonal antibody, FlaA often formed a doublet, with the major band migrating to approximately $38 \mathrm{kD}$ and the minor band at $37 \mathrm{kD}$. With the monoclonal antibody, approximately $15 \mu \mathrm{g}$ protein lysate loaded in the gel lane were necessary to detect FlaA in MC-1. No FlaA in MC-1 was seen with the polyclonal antiserum under these same conditions. Quantitative immunoblotting analysis indicated that the amount of FlaA in MC-1 was approximately $13 \%$ of the wild-type (Figure 4b). To further test if this inhibition of FlaA accumulation was specifically related to flaB::kan, we tested the flaB::kan complemented strain MS17. This strain is wavy, motile, and has PFs (61). We found that the level of FlaA in MS17 was equivalent to that of the wild-type (Figure 4b). These results suggest that the flaB::kan mutation specifically decreases FlaA accumulation in B. burgdorferi. 


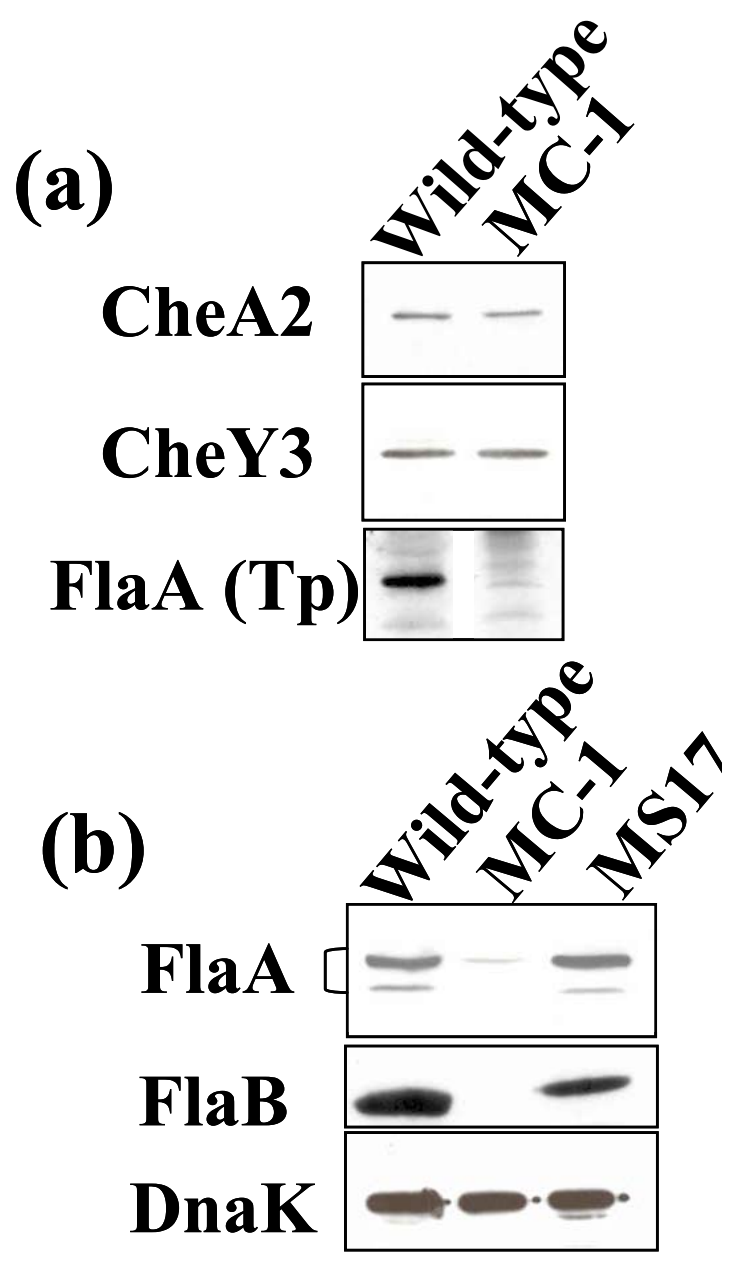

Figure 4. Effect of flaB::kan on proteins encoded by genes in the flaA operon.

(a) Western blot analysis of wild-type and MC-1. FlaA was the probed with polyclonal $T$. pallidum FlaA antiserum (Tp). (b) Western blot probed with monoclonals anti-FlaA, FlaB, and DnaK to the wild type, MC-1, and the complemented strain MS-17. DnaK was used as an internal control. For FlaA, FlaB, and DnaK detection in the wild-type and MS-17, approximately $10 \mu \mathrm{g}$ of cell lysate were loaded into each lane. For MC-1, $20 \mu \mathrm{g}$ of cell lysate were loaded into the lane for FlaA, and $10 \mu \mathrm{g}$ for DnaK. The apparent sizes of the proteins are the following (kD): CheA2, 98; CheY3, 11; FlaA, 38; DnaK, 72. 
Transcription of flaA in MC-1. One mechanism that could explain the reduced FlaA protein levels in the flaB::kan mutant is inhibition of flaA transcription. To test for this possibility, mRNA levels were measured for the wild-type and flaB::kan mutant using both quantitative RT-PCR and RNase protection assays. 16S rRNA was used as a control in both assays. Quantitative RT-PCR results indicated that the level of flaA transcript in MC-1 was similar to or slightly greater than that of the wild-type (Figure 5). Furthermore, RNase protection results yielded phospholmager units for the wild-type and MC-1 for flaA of 2150 and 2020, and for 16 S rRNA, 1175 and 1015 respectively. These results taken together reveal that the flaB::kan mutation did not negatively influence flaA mRNA synthesis. The results are also consistent with the lack of inhibition of downstream gene expression in this operon in MC-1 as exemplified by CheA2 and CheY3 (Figure 4a). 


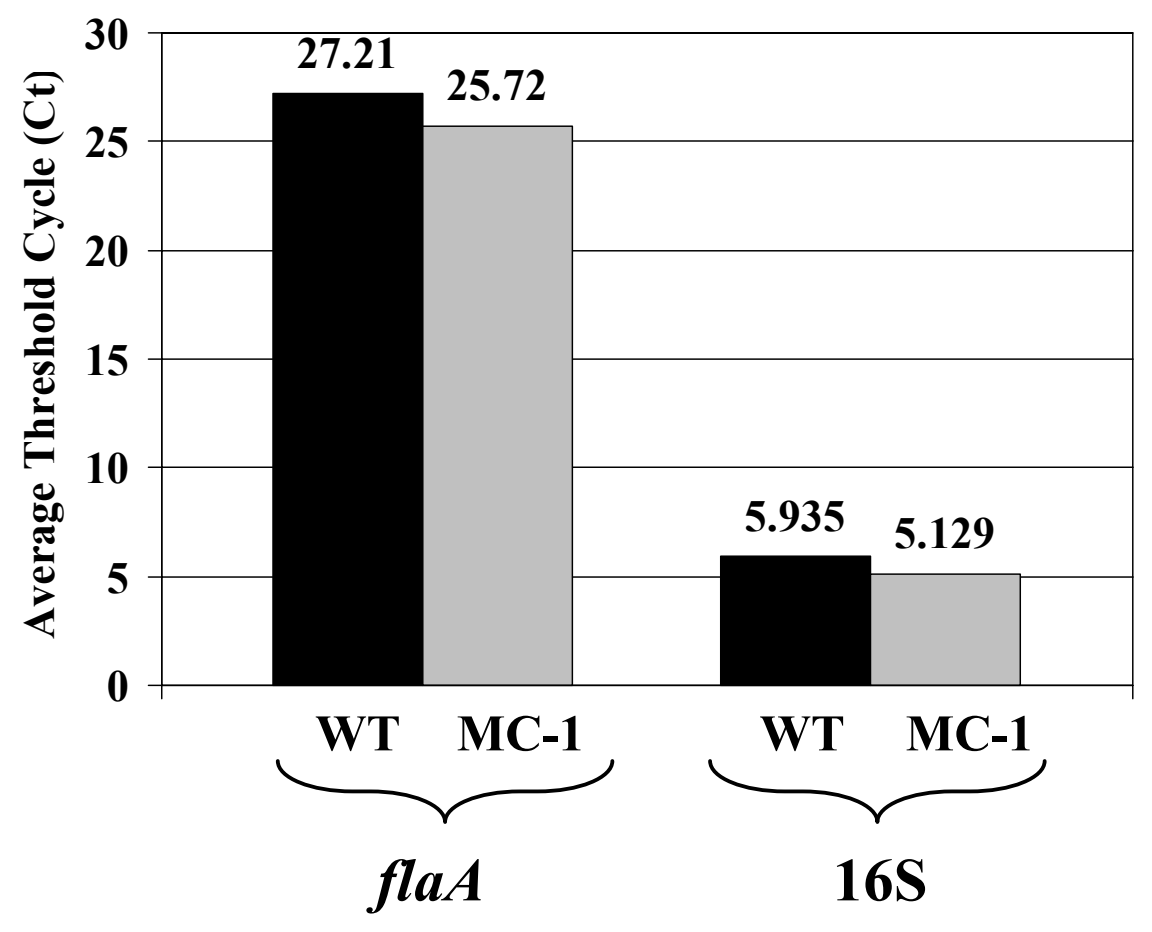

Figure 5. Detection and quantification of relative amounts of fla $A$ transcript in wild-type and MC-1 strain using real-time RT-PCR.

Average threshold cycle (Ct) is calculated from duplicate samples in each experiment. 
Post-transcriptional control of FlaA. Two explanations are evident with respect to the lack of FlaA protein detected in MC-1. One hypothesis states that FlaA synthesis is controlled at the translational level. For example, perhaps FlaB is an activator for translation of FlaA. Alternatively, perhaps without FlaB, FlaA is rapidly degraded. To test for possible turnover, spectinomycin was added to growing cells, and the stability of FlaA was monitored by Western blot analysis (Figure 6). Spectinomycin at $100 \mu \mathrm{g} / \mathrm{ml}$ was found to rapidly inhibit protein synthesis and growth, and the cells remained intact for several hours. DnaK was used as a control, as transcription of this protein has been previously shown to be relatively constant under different growth conditions (56). We found that the level of DnaK in spectinomycin treated cells was stable in both the wild-type and MC-1 for as long 12 hours (Figure 6). Similar results were found with respect to FlaA in the wild-type. However, the level of FlaA in MC-1 showed approximately a $50 \%$ decrease by 2 hours. By 12 hours, there still was a trace of FlaA present. These results indicate that FlaA is degraded in the MC-1 with a half-life of approximately 2 hours. 


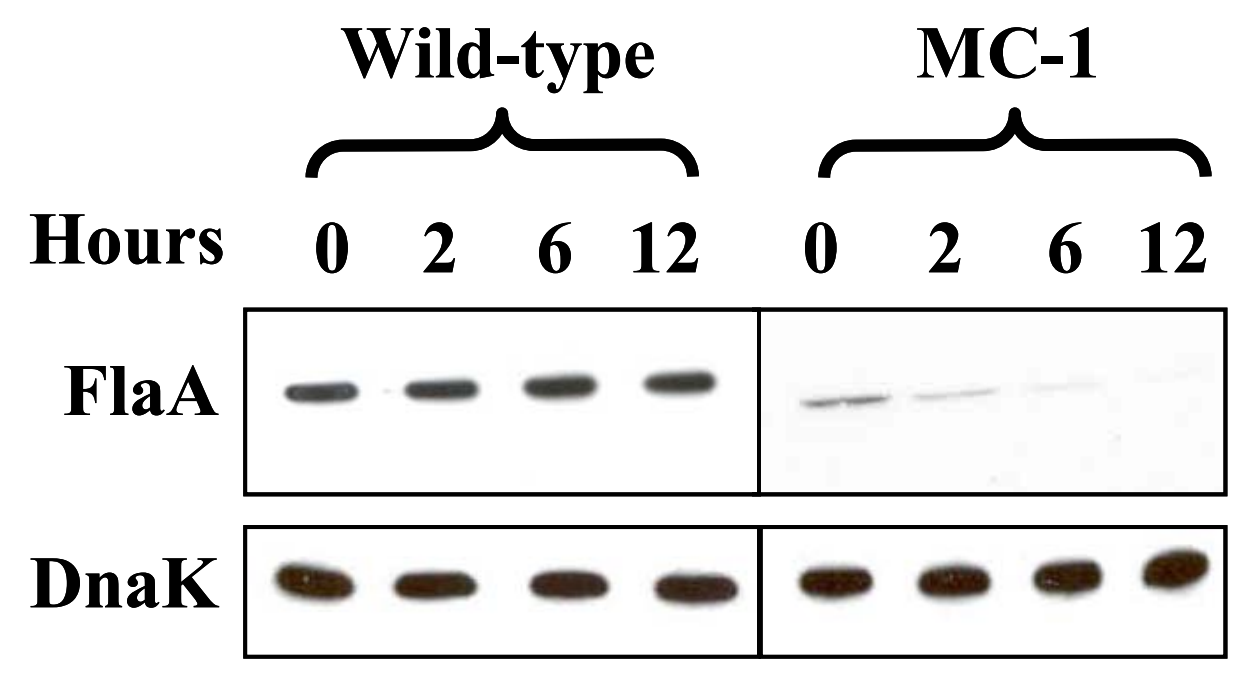

Figure 6. Degradation of FlaA.

Stability of FlaA was determined by adding spectinomycin $(100 \mu \mathrm{g} / \mathrm{ml}$ culture) to growing $B$. burgdorferi cells. At different time points, samples were subjected to Western blotting with monoclonal anti-FlaA. DnaK was used as an internal control. Approximately $5 \mu \mathrm{g}$ of lysate were loaded into each lane for the wild-type. For MC-1, approximately $20 \mu \mathrm{g}$ was loaded for detection of FlaA, and $5 \mu \mathrm{g}$ for DnaK. 


\section{Discussion}

Motility is energetically costly to $B$. burgdorferi. We found that the contribution of the major PF filament protein FlaB to the organism is substantial; it constitutes between 10-14 per cent of the total cellular protein. Furthermore, at least 5 per cent of the genome is dedicated to motility and chemotaxis (18). Specifically, approximately 42 open reading frames (ORFs) homologous to motility genes of other bacteria have been identified; this estimate includes the multiple homologs of certain chemotaxis (cheY, cheA, cheW, cheR, cheB) and flagellar genes (fliG). Targeted mutagenesis of many of these genes indicate that they function in motility and chemotaxis (M. Motaleb, C. Li, M. Sal, R. Bakker, and N. Charon, unpublished; (40)). In addition, eight more ORFs of undetermined function map within the major motility operons. Because of their location, these ORFs are also likely to be involved in motility and chemotaxis. Thus, we estimate that approximately 50 of the total 1000 ORFs encoded by the $B$. burgdorferi chromosome are involved in these functions.

We found that there was considerably less FlaA in $B$. burgdorferi than FlaB, with FlaA contributing less than 0.5 per cent of the total cellular protein. The function of FlaA in spirochete motility is presently unknown. In several spirochete species, FlaA forms a protein sheath around the FlaB core $(9,11,39$, 41). Null mutants in flaA in $B$. hyodysenteriae have been characterized $(41,58)$. These mutants lack the flagellar protein sheath, have an altered PF helical shape, but they still retain motility (41). Microscopic observations and swarm plate assays indicate that these fla $A$ mutants of $B$. hyodysenteriae are less motile 
than the wild-type $(41,58)$. In $B$. burgdorferi, no sheath is evident in electron microscopic observation (M. Motaleb and N. Charon, unpublished). As shown by immunoprecipitation, $\mathrm{FlaA}$ associates with $\mathrm{FlaB}$, confirming that it is a flagellar protein. In contrast to $B$. hyodysenteriae (41), FlaA did not influence the helical shape of the PFs from B. burgdorferi. Preliminary results with a flaA::kan mutant yielded a PF shape identical to the wild type (M. Motaleb and N. Charon, unpublished). Future experiments will focus on localizing FlaA on the PF filament using immunogold technology.

We found that FlaA in $B$. burgdorferi often migrated as a doublet by Western blot analysis (Figure 4b). These doublets were most commonly observed when large amounts of protein were loaded in the gels. The major protein was approximately $38 \mathrm{kD}$ in size, which is consistent with our published results with strain 212 and the results of others $(23,27)$. The minor protein of approximately $37 \mathrm{kD}$ was often not seen (Figures 1,2, and 6), and we could only detect it by Western blotting. The minor $37 \mathrm{kD}$ band has not been previously reported $(23,27)$. Several possible explanations could account for the lack of previously detecting the minor FlaA protein. One possibility is differences with anti-FlaA reagents. In our previous studies, we used a heterologous polyclonal antibody to T. pallidum FlaA (23). As shown in Figure 4, the minor protein was not detected using this antiserum; it was only detected using the monoclonal antiFlaA of B. burgdorferi. Furthermore, when we tested strain 212 using the monoclonal antibody, the Western blot pattern was identical to strain B31 (M. 
Motaleb and N. Charon, unpublished). Thus, the $37 \mathrm{kD}$ band is not strain specific. In addition, this minor protein was not detected by Gilmore et al., who first obtained and analyzed the FlaA monoclonal antibody (27). One possible explanation is that these investigators used the less sensitive colorimetric assay for immunostaining. Because the $37 \mathrm{kD}$ protein is in relatively small amounts, it may have gone undetected (27). We found that prolonged incubation of cell lysates at $23^{\circ} \mathrm{C}$ or $37^{\circ} \mathrm{C}$ did not change the ratio of the two FlaA bands; thus, it is unlikely that the $37 \mathrm{kD}$ protein is the result of protein degradation (M. Motaleb and $\mathrm{N}$. Charon, unpublished). Both proteins are likely to be encoded by flaA, as a flaA::kan mutant lacks both proteins, but still synthesizes FlaB at an amount similar to the wild-type (M. Motaleb and N. Charon, unpublished). Our preliminary experiments to characterize the minor band by mass spectroscopy have been unsuccessful. It will be interesting in future experiments to sort out the relationship of the two proteins. Several lines of evidence suggest that the Nterminus of the FlaA is cleaved by signal peptidase I $(14,21,23,27)$, and that this protein is also glycosylated (23). Perhaps the lower molecular weight form is unglycosylated FlaA. Alternatively, others have shown that the carboxy-terminal end of certain outer membrane proteins of $B$. burgdorferi is cleaved (52), and thus the small molecular weight protein could be the result of specific protease digestion.

We examined the effects of an insertion mutation in $f l a B$ on the synthesis of other motility proteins. Because B. burgdorferi lacks sigma dependent cascade 
control of flagellar gene regulation $(2,11,21,22,25,42)$, we could not predict how a flaB mutation would influence the expression of other motility genes. Western blot analysis indicates that 8 of 9 putative chemotaxis and motility proteins tested had unaltered levels of accumulation compared to the wild-type. Of those tested, the only gene product found to be significantly reduced was FlaA at a level of approximately 13 per cent of the wild-type (Figure 4b). In E. coli and S. enterica strain Typhimurium, mutants in fliC, which encodes the only protein found in the filament, do not effect the expression of other flagellar genes (15). Thus, the results obtained are similar in some respects to those of $E$. coli. However, several species of bacteria have multiple filament proteins. For example, Caulobacter crescentus and Vibrio parahaemolyticus have six (17, 4749), B. hyodysenteriae has four $(42,58)$, and Helicobacter pylori, H. mustelae, and Campylobacter jejuni have two $(36,62,65)$. In these species, mutants in the genes that encode specific filament proteins still synthesize the other filament proteins at levels close to those of wild type $(36,41,42,49,62)$. Thus, $B$. burgdorferi is different from these other species of bacteria, as flaB::kan did decrease the amount of FlaA.

We investigated the basis of FlaA inhibition in the flaB::kan mutant. The results indicated that there was no change in the amount of fla $A$ message. These results suggest that the inhibition of FlaA in the $\mathrm{MC}-1$ is post-transcriptional. Post-transcriptional control of flagella gene expression has been noted in other species. For example, in S. enterica serovar Typhimurium, this control is coupled 
to the secretion and assembly of basal body proteins and flagellin $(2,7,37,38)$. In addition, the membrane protein FIK regulates translation of the flagellumspecific anti-sigma factor FlgM in response to flagellar ring assembly (38). In C. crescentus, synthesis of both FljK and FljL flagellar filament proteins have been shown to be under translational control at specific checkpoints in flagellar assembly $(3,49)$. Recently, Limberger et al. isolated a mutant in tap1 in Treponema denticola (44). tap1 is a homolog to the flagellar hook assembly gene fliK found in other bacteria. This mutant failed to make flagellar filament FlaB proteins, but RT-PCR results indicated that flaB continued to be transcribed. Because the relative amount of flaB mRNA was not determined in tap1, it is unclear whether the level of flaB mRNA in the mutant achieved wild type levels.

There are two likely explanations for the inhibition of FlaA accumulation in the FlaB mutant. First, FlaA could be degraded by cell proteases. Spectinomycin inhibition results indicate that, whereas this protein is stable in the wild-type, the small amount detected in the flaB::kan mutant was degraded with a half-life of approximately 2 hours. This turnover rate relative to the 8-10 hour generation time would readily explain the small amount of FlaA detected in the flaB mutant. To our knowledge, these results are the first evidence for protein turnover in $B$. burgdorferi. Degradation of FlaA in this mutant could be related to the complicated assembly of spirochete flagellar filaments. Specifically, FlaB has no signal sequence and is excreted into the periplasmic space by a type III secretory pathway in a manner similar to other bacterial flagellin $(11,13,21,46)$. On the 
other hand, FlaA of $B$. burgdorferi and other spirochetes contain a signal peptidase I cleavage site near its $\mathrm{N}$-terminus (21). Several lines of evidence indicate that it reaches the periplasmic space by the type II pathway $(13,14,21$, $23,27,42)$. Both proteins evidently form a complex in the periplasmic space for filament formation. Perhaps in the absence of FlaB, FlaA is more vulnerable to protease digestion. In other systems, proteins that comprise a complex but fail to pair with their partners are often unstable and readily degradable, e.g. RcsA and RcsB in E. coli (30). This hypothesis is further supported by our finding that among the downstream genes in the flaA operon, the amount of CheA2 and CheY3 were unaltered in MC-1 as compared to the wild-type (Figure 4a).

Another possible explanation is translational control of FlaA. For example, perhaps FlaB or another factor acts as a positive effector for translation of the FlaA message. This type of control has been noted in other systems, such as the Com protein stimulating translation of mom mRNA in bacteriophage Mu (64). Genomic analysis of $B$. burgdorferi indicates that it has only a few twocomponent signal transduction systems, and obvious operator-repressor systems are not evident (18). Recent evidence indicates that RpoN and RpoS are both involved in the regulation of gene expression in tick and mammalian hosts (33). Perhaps $B$. burgdorferi evolved specific adaptive strategies for these hosts, and consequently shed many of the control mechanisms found in other bacteria. Along these lines, several other genes (e.g. Bbk2.10 and elpA.1, flaA and flaB in a flgE mutant) have been identified in B. burgdorferi for which there was 
abundant messenger RNA present, but the encoded protein was undetectable or markedly decreased ((31); Sal et al., Abstr. Bacterial Locomotion and Signal Transduction Meeting VII). The results presented here and elsewhere do not allow us to differentiate which type of mechanism (translational control vs degradation) is occurring with respect to FlaA regulation and these other proteins; perhaps both are occurring simultaneously. To precisely sort this out, rapid pulse-labeling experiments would be helpful. However, because of the rich nutrient requirements of $B$. burgdorferi, our attempt to use this approach have so far been unsuccessful.

\section{ACKNOWLEDGMENTS}

We thank A. Barbour, D. Blair, J. Benach, F. Cabello, J. Carroll, B. Johnson, P. Matsumura, S. Norris, and the late R. Macnab for kindly sharing strains, monoclonal antibodies, and antisera. We also thank T. Elliot, M. Hirsch, S. Minnich, J. Olson, E. Rucks, R. Bakker, C. Li, and D. Yelton for suggestions and critical comments on this manuscript. This research was supported by U.S. Public Health Service Grants Al29743 to N.W.C., and American Heart Association Grant 0365225B to M.A.M. 


\section{References}

1. Aizawa, S. I., I. B. Zhulin, L. M. Marquez-Magana, and G. W. Ordal. 2001. Chemotaxis and Motility, p. 437-452. In A. L. Sonenshein, J. A. Hoch, and R. Losick (eds.), Bacillus subtilis and its closest relatives: from genes to cells. ASM Press, Washington D.C.

2. Aldridge, P. and K. T. Hughes. 2002. Regulation of flagellar assembly. Curr. Opin. Microbiol. 5:160-5.

3. Anderson, D. K. and A. Newton. 1997. Posttranscriptional regulation of Caulobacter flagellin genes by a late flagellum assembly checkpoint. J. Bacteriol. 179:2281-2288.

4. Barbour, A. G. and S. F. Hayes. 1986. Biology of Borrelia species. Microbiol. Rev. 50:381-400.

5. Barbour, A. G., S. F. Hayes, R. A. Heiland, M. E. Schrumpf, and S. L. Tessier. 1986. A Borrelia-specific monoclonal antibody binds to a flagellar epitope. Infect. Immun. 52:549-54.

6. Berg, H. C. 2003. The rotary motor of bacterial flagella. Annu. Rev. Biochem. 72:19-54.

7. Bonifield, H. R., S. Yamaguchi, and K. T. Hughes. 2000. The flagellar hook protein, FlgE, of Salmonella enterica serovar typhimurium is posttranscriptionally regulated in response to the stage of flagellar assembly. J. Bacteriol. 182:4044-50.

8. Bono, J. L., A. F. Elias, J. J. Kupko, B. Stevenson, K. Tilly, and P. Rosa. 2000. Efficient targeted mutagenesis in Borrelia burgdorferi. J. Bacteriol. 182:2445-52.

9. Brahamsha, B. and E. P. Greenberg. 1988. Biochemical and cytological analysis of the complex periplasmic flagella from Spirochaeta aurantia. J. Bacteriol. 170:4023-4032.

10. Carroll, J. A., N. El-Hage, J. C. Miller, K. Babb, and B. Stevenson. 2001. Borrelia burgdorferi RevA antigen is a surface-exposed outer membrane protein whose expression is regulated in response to environmental temperature and $\mathrm{pH}$. Infect. Immun. 69:5286-93.

11. Charon, N. W. and S. F. Goldstein. 2002. Genetics of motility and chemotaxis of a facinating group of bacteria: The Spirochetes. Annu. Rev. Genet. 36:47-73. 
12. Charon, N. W., S. F. Goldstein, S. M. Block, K. Curci, J. D. Ruby, J. A. Kreiling, and R. J. Limberger. 1992. Morphology and dynamics of protruding spirochete periplasmic flagella. J. Bacteriol. 174:832-40.

13. Charon, N. W., E. P. Greenberg, M. B. Koopman, and R. J. Limberger. 1992. Spirochete chemotaxis, motility, and the structure of the spirochetal periplasmic flagella. Res. Microbiol. 143:597-603.

14. Charon, Y. G. a. N. W. 1997. FlaA, a Putative Flagellar Outer Sheath Protein, Is Not and Immunodominant Antigen Assoicated with Lyme Disease. Infect. Immun. 65:2992-2995.

15. Chilcott, G. S. and K. T. Hughes. 2000. Coupling of flagellar gene expression to flagellar assembly in Salmonella enterica serovar typhimurium and Escherichia coli. Microbiol. Mol. Biol. Rev. 64:694-708.

16. Coleman, J. L., R. C. Rogers, and J. L. Benach. 1992. Selection of an escape variant of Borrelia burgdorferi by use of bactericidal monoclonal antibodies to OspB. Infect. Immun. 60:3098-104.

17. Ely, B., T. W. Ely, W. B. Crymes, Jr., and S. A. Minnich. 2000. A family of six flagellin genes contributes to the Caulobacter crescentus flagellar filament. J. Bacteriol. 182:5001-5004.

18. Fraser, C. M., S. Casjens, W. M. Huang, G. G. Sutton, R. Clayton, R. Lathigra, O. White, K. A. Ketchum, R. Dodson, E. K. Hickey, M. Gwinn, B. Dougherty, J. F. Tomb, R. D. Fleischmann, D. Richardson, J. Peterson, A. R. Kerlavage, J. Quackenbush, S. Salzberg, M. Hanson, R. van Vugt, N. Palmer, M. D. Adams, J. Gocayne, J. C. Venter, and et al. 1997. Genomic sequence of a Lyme disease spirochaete, Borrelia burgdorferi. Nature 390:580-6.

19. Fraser, C. M., T. Hirano, H. U. Ferris, L. L. Devgan, M. Kihara, and R. M. Macnab. 2003. Substrate specificity of Type III flagellar protein export in Salmonella is controlled by subdomain interactions in FlhB. Mol. Microbiol. 48:1043-1057.

20. Gassmann, G. S., E. Jacobs, R. Duetzmann, and Gobel U.B. 1991. Analysis of the Borrelia burgdorferi GeHo fla gene and antigenic characterization of its gene product. J. Bacteriol. 173:1452-1459.

21. Ge, Y. and N. W. Charon. 1997. An Unexpected flaA Homolog Is Present and Expressed in Borrelia burgdorferi. J. Bacteriol. 179:552-556.

22. Ge, Y. and N. W. Charon. 1997. Molecular characterization of a flagellar/chemotaxis opernon in the spriochete Borrelia burgdorferi. FEMS Microbiol. Lett. 152:425-431. 
23. Ge, Y., C. Li, L. Corum, C. A. Slaughter, and N. W. Charon. 1998. Structure and expression of the FlaA periplasmic flagellar protein of Borrelia burgdorferi. J. Bacteriol. 180:2418-25.

24. Ge, Y., I. G. Old, I. Saint Girons, and N. W. Charon. 1997. Molecular characterization of a large Borrelia burgdorferi motility operon which is initiated by a consensus sigma70 promoter. J. Bacteriol. 179:2289-99.

25. Ge, Y., I. G. Old, I. Saint Girons, and N. W. Charon. 1997. Molecular characterization of a large Borrelia burgdorferi motility operon which is initiated by a consensus sigma70 promoter. J. Bacteriol. 179:2289-99.

26. Ge, Y. and N. W. Charon. 1997. Identification of a large motility operon in Borrelia burgdorferi by semi-random PCR chromosome walking. Gene 189:195-201.

27. Gilmore, R. D., R. L. Murphree, A. M. James, S. A. Ullivan, and B. J. Johnson. 1999. The Borrelia burgdorferi 37-kiodalton immunoblot band (p37) used in serodiagnosis of early Lyme disease is the flaA gene product. J. Clin. Microbiol. 37:548-552.

28. Goldstein, S. F., K. F. Buttle, and N. W. Charon. 1996. Structural analysis of the Leptospiraceae and Borrelia burgdorferi by high-voltage electron microscopy. J. Bacteriol. 178:6539-45.

29. Goldstein, S. F., N. W. Charon, and J. A. Kreiling. 1994. Borrelia burgdorferi swims with a planar waveform similar to that of eukaryotic flagella. Proc. Natl. Acad. Sci. USA 91:3433-7.

30. Gottesman, S. and M. R. Maurizi. 1992. Regulation by proteolysis:energy-dependent proteases and their targets. Microbiol. Rev. 56:592-621.

31. Hefty, P. S., S. E. Jolliff, M. J. Caimano, S. K. Wikel, J. D. Radolf, and D. R. Akins. 2001. Regulation of OspE-related, OspF-related, and Elp lipoproteins of Borrelia burgdorferi strain 297 by mammalian host-specific signals. Infect. Immun. 69:3618-3627.

32. Hovind-Hougen, K. 1984. Ultrastructure of spirochetes isolated from Ixodes ricinus and Ixodes dammini. Yale J. Biol. Med. 57:543-8.

33. Hubner, A., X. Yang, D. M. Nolen, T. G. Popova, F. C. Cabello, and M. V. Norgard. 2001. Expression of Borrelia burgdorferi OspC and DbpA is controlled by a RpoN-RpoS regulatory pathway. Proc. Natl. Acad. Sci. USA 98:12724-9. 
34. Johansson, J., A. Labigne, and S. Suerbaum. 2002. An RNA thermosensor expression of virulence genes in Listeria monocytogenes. Cell 110:551.

35. Johnson, R. C. S. G. P. H. F. W. S. A. G. a. B. D. J. 1984. Borrelia burgdorferi sp. nov.: Etiologic Agent of Lyme Disease. International Systematic Bacteriology 496-497.

36. Josenhans, C., A. Labigne, and S. Suerbaum. 1995. Comparative ultrastructural and functional studies of Helicobacter pylori and Helicobacter mustelae flagellin mutants: both flagellin subunits, FlaA and FlaB, are necessary for full motility in Helicobacter species. J. Bacteriol. 177:3010-20.

37. Karlinsey, J. E., J. Lonner, K. L. Brown, and K. T. Hughes. 2000. Translation/secretion coupling by type III secretion systems. Cell 102:48797.

38. Karlinsey, J. E., H. C. T. Tsui, M. E. Winkler, and K. T. Hughes. 1998. Flk couples flgM translation to flagellar ring assembly in Salmonella typhimurium. J. Bacteriol. 180:5384-5397.

39. Koopman, M. B., O. S. de Leeuw, B. M. van der Zeijst, and J. G. Kusters. 1992. Cloning and DNA sequence analysis of a Serpulina (Treponema) hyodysenteriae gene encoding a periplasmic flagellar sheath protein. Infect. Immun. 60:2920-2925.

40. Li, C., R. G. Bakker, M. A. Motaleb, M. L. Sartakova, F. C. Cabello, and N. W. Charon. 2002. Asymmetrical flagellar rotation in Borrelia burgdorferi nonchemotactic mutants. Proc. Natl. Acad. Sci. USA 99:6169-74.

41. Li, C., L. Corum, D. Morgan, E. L. Rosey, T. B. Stanton, and N. W. Charon. 2000. The spirochete FlaA periplasmic flagellar sheath protein impacts flagellar helicity. J. Bacteriol. 182:6698-706.

42. Li, C., A. Motaleb, M. Sal, S. F. Goldstein, and N. W. Charon. 2000. Spirochete periplasmic flagella and motility. J. Mol. Microbiol. Biotechnol. 2:345-54.

43. Limberger, R. J. and N. W. Charon. 1986. Treponema phagedenis has at least two proteins residing together on its periplasmic flagella. J. Bacteriol. 166:105-12.

44. Limberger, R. J., L. L. Slivienski, J. Izard, and W. A. Samsonoff. 1999. Insertional inactivation of the Treponema denticola tap1 results in a nonmotile mutant with elongated flagellar hooks. J. Bacteriol. 181:37433750 . 
45. Lux, R., A. Moter, and W. Shi. 2000. Chemotaxis in pathogenic spirochetes: directed movement toward targeting tissues? J. Mol. Microbiol. Biotechnol. 2:355-64.

46. Macnab, R. M. 1996. Flagella and Motility, p. 123-145. In F. C. Neidhardt, R. Curtiss III, J. L. Ingraham, C. C. Lin, K. B. Low, B. Magasanik, W. S. Reznikoff, M. Riley, M. Schaechter, and H. E. Umbarger (eds.), Escherichia coli and Salmonella typhimurium:cellular and molecular biology. ASM Press, Washington D.C.

47. McCarter, L. L. 1995. Genetic and Molecular characterizationof the polar flagellum of Vibrio parahaemolyticus. J. Bacteriol. 177:1595-1609.

48. McCarter, L. L. 2001. Polar Flagellar Motility of the Vibrionaceae. Microbiol. Mol. Biol. Rev. 65:445-462.

49. Minnich, S. A., N. Ohta, N. Taylor, and A. Newton. 1988. Role of the 25, 27-, and 29-kilodalton flagellins in Caulobacter crescentus cell motility: method for construction of deletion and Tn5 insertion mutants by gene replacement. J. Bacteriol. 170:3953-3960.

50. Motaleb, M. A., L. Corum, J. L. Bono, A. F. Elias, P. Rosa, D. S. Samuels, and N. W. Charon. 2000. Borrelia burgdorferi periplasmic flagella have both skeletal and motility functions. Proc. Natl. Acad. Sci. USA 97:10899-904.

51. Neidhardt, F. C. 1987. Escherichia coli and Salmonella typhimurium: cellular and molecular biology, p. 3-6. American Society for Microbiology, Washington D.C.

52. Noppa, L., Y. Ostberg, C. W. Lawrence, and S. Bergstrèom. 2001. P13, an integral membrane protein of Borrelia burgdorferi, is C-terminally processed and contains surface exposed domains. Infect. Immun. 69:3323-3334.

53. Norris, S. J. 1993. Polypeptides of Treponema pallidum: progress toward understanding their structural, functional, and immunologic roles. Treponema Pallidum Polypeptide Research Group. Microbiol. Rev. 57:750-79.

54. Norris, S. J., N. W. Charon, R. G. Cook, M. D. Fuentes, and R. J. Limberger. 1988. Antigenic relatedness and $\mathrm{N}$-terminal sequence homology define two classes of periplasmic flagellar proteins of Treponema pallidum subsp. pallidum and Treponema phagedenis. J. Bacteriol. 170:4072-4082. 
55. Nuttall, P. A., G. C. Paesen, C. H. Lawrie, and H. Wang. 2000. Vectorhost interactions in disease transmission. J. Mol. Microbiol. Biotechnol. 2:381-386.

56. Ojaimi, C., C. Brooks, S. Casjens, P. Rosa, A. Elias, A. Barbour, A. Jasinskas, J. Benach, L. Katona, J. Radolf, M. Caimano, J. Skare, K. Swingle, D. Akins, and I. Schwartz. 2003. Profiling of TemperatureInduced Changes in Borrelia burgdorferi Gene Expression by Using Whole Genome Arrays. Infect. Immun. 71:1689-1705.

57. Pfaffl, M. W. 2001. A new mathematical model for relative quantities in real-time RT-PCR. Nucleic Acids Res. 29:2003-2007.

58. Rosey, E. L., M. J. Kennedy, D. K. Petrella, R. G. Ulrich, and R. J. Yancey, Jr. 1995. Inactivation of Serpulina hyodysenteriae flaA1 and flaB1 periplasmic flagellar genes by electroporation-mediated allelic exchange. J. Bacteriol. 177:5959-5970.

59. Ruby, J. D., H. Li, H. Kuramitsu, S. J. Norris, S. F. Goldstein, K. F. Buttle, and N. W. Charon. 1997. Relationship of Treponema denticola periplasmic flagella to irregular cell morphology. J. Bacteriol. 179:1628-35.

60. Sadziene, A., D. D. Thomas, V. G. Bundoc, S. C. Holt, and A. G. Barbour. 1991. A flagella-less mutant of Borrelia burgdorferi. Structural, molecular, and in vitro functional characterization. J. Clin. Invest 88:82-92.

61. Sartakova, M. L., E. Y. Dobrikova, M. A. Motaleb, H. P. Godfrey, N. W. Charon, and F. C. Cabello. 2001. Complementation of a nonmotile flaB mutant of Borrelia burgdorferi by chromosomal Integration of a plasmid containing a wild-type flaB allele. J. Bacteriol. 183:6558-64.

62. Wassenaar, T. M., N. M. Bleumink-Pluym, and B. A. van der Zeijst. 1991. Inactivation of Camplyobacter jejuni flagellin genes by homologous recombination demonstrates that flaA, but not flaB, is required for invasion. EMBO J. 10:2055-2061.

63. Wilson, D. R. and T. J. Beveridge. 1993. Bacterial flagellar filaments and their component flagellins. Can. J. Microbiol. 39:451-472.

64. Wulczyn, F. G. and R. Kahmann. 1991. Translational stimulation:RNA sequence and structure requirements for binding of Com protein. Cell 65:259-269.

65. Yao, V. J. and J. L. Spudich. 1992. Primary structure of an archaebacterial transducer, a methyl-accepting protein associated with sensory rhodopsin I. Proc. Natl. Acad. Sci. USA 89:11915-9. 


\section{Chapter 3. Post-transcriptional flagellin regulation in a}

periplasmic flagellar hook mutant of Borrelia burgdorferi

Manuscript in preparation:

Melanie S. Sal, Md. A. Motaleb, and Nyles W. Charon 


\section{Abstract}

Borrelia burgdorferi is a wave-like, motile spirochete that causes Lyme disease.

In $B$. burgdorferi, motility is provided by bundles of between 7 and 11 periplasmic flagella (PFs) attached near the ends of the cell that extend inward along the cell cylinder beneath an outer membrane sheath. These PFs are similar in structure to flagella from other bacteria. However, the control of B. burgdorferi PF synthesis differs from flagella synthesis in other bacteria, as the transcription factors sigma-28 and sigma-54 are not involved in its regulation. Previous results with a flaB filament mutant have shown that PFs are involved in both cell morphology and motility. To further test this finding and to gain insight into flagellar gene regulation in this species, we inactivated the gene encoding the PF hook protein. Cells from the isolated transformant colony SC-1 were rod-shaped and non-motile. We investigated the effects of flgE::kan mutation on the regulation of PF proteins by Western analysis. Although most motility and chemotaxis genes were expressed at wild-type levels, SC-1 cells had markedly decreased levels of PF filament proteins FlaA and FlaB. Quantitative RT-PCR revealed that the reduction of flagellin proteins in SC-1 was not the result of a reduction in flaA or flaB message. Using spectinomycin to inhibit protein synthesis, we found that the small amount of FlaA in SC-1 was turned over. In contrast, the amount of FlaB was stable in the presence of spectinomycin. Our results indicate that the inhibition of flagellar hook protein synthesis negatively impacts both FlaA and FlaB post-transcriptionally. Moreover, our results strongly support translational control of FlaB, and possibly FlaA. 


\section{Introduction}

Borrelia burgdorferi, the causative agent of Lyme disease, is a spirochete with characteristic wave-like cell morphology (22). As a result of the unusual morphology, this and other spirochete species have unique modes of motility that provide them with a highly specialized ability to traverse viscous gel-like material $(6,9,23,23,30)$. This unique swimming ability is believed to be advantageous for the spirochetes to penetrate host connective tissue $(36,37)$. Evidence supporting the idea that motility is important in host invasion comes from the analysis of a non-motile, flagella-less isolate of $B$. burgdorferi that was less able to penetrate endothelial cells than the wild type (45). In addition to studies in $B$. burgdorferi, the importance of motility as a virulence factor has been implicated in spirochetes including Treponema denticola, Brachyspira hyodysenteriae, and Borrelia garnini $(36,43,45,48)$

Motility in B. burgdorferi is provided by bundles of between 7 and 11 periplasmic flagella (PFs) subterminally attached near the ends of the cell that extend inward along the cell cylinder beneath an outer membrane sheath $(5,21$, 39). Rotation of the PFs in opposite directions, as viewed along the PF to where it inserts in the cell cylinder, causes propagation of backward moving waves along the cell body that propel the bacteria forward $(9,10,21,22,35)$. A given PF is composed of a basal body, hook, and filament, and is similar in structure to the flagellum of other bacteria (49). However, in contrast to single component filaments (FliC) or filaments with multiple FlaB subunits found in other 
spirochetes, $B$. burgdorferi PF filaments are composed of a single major flagellin FlaB and a minor flagellin FlaA $(17,39,40)$. The location of FlaA in $B$. burgdorferi is still under investigation, and the reason for multiple flagellin subunits in spirochete PF filaments is unknown. The PFs in B. burgdorferi also have an interesting role in influencing overall cell shape. Sadziene (45) observed that a spontaneous non-motile, flagella-less isolate of $B$. burgdorferi had an altered cell shape relative to wild-type cells. This mutant was later described as rod-shaped (22). In agreement with previous findings, recent analysis of a targeted mutant in $f l a B$ revealed that PFs are critical for providing the characteristic wave-like morphology in addition to their essential role in motility $(39,47)$.

Flagellum synthesis, as described in Escherichia coli and Salmonella enterica serovar Typhimurium, is a finely orchestrated succession of motility gene expression and protein assembly that requires tight regulation. In enteric bacteria, flagella synthesis is regulated by a cascade of transcriptional events involving the ordered expression of class I, class II, and class III motility genes. Expression of class I genes, which comprise the master operon, directs the transcription of class II genes $(1,11)$. Class II genes are those that encode structural proteins involved with the early stages of flagella synthesis and regulatory proteins controlling the expression of late stage class III flagella and chemotaxis genes $(1,11)$. Among the class II regulatory genes are fliA, encoding the motility specific transcription factor sigma-28, and flgM, encoding 
the anti-sigma-28 factor. FlgM and sigma-28 remain bound as a complex in the cytoplasm during basal body and hook synthesis. Upon completion of the hook, this structure provides an export route for the anti-sigma factor $\operatorname{FlgM}(29,34)$. As FlgM exits the cell, unbound sigma-28 is free to initiate the transcription of late genes, including fliC $(1,11)$. In the event that an intact hook is not formed or is not functional, FlgM cannot escape and remains associated with sigma-28 to prevent the transcription of flagellin genes (29). Thus, completion of the basal body-hook structure acts as a general assembly checkpoint in the regulation of flagella synthesis, specifically where the flagellin genes are controlled transcriptionally in response to the state of hook completion.

Although S. enterica flagella regulation and synthesis has been extensively studied, not all bacterial species regulate their flagella synthesis in the same manner. For example, Caulobacter crescentus regulates its flagella synthesis by a hierarchy of four classes of flagella genes. The Class I product CtrA is required for expression of Class II genes (50). Of the Class II gene products, including those for the basal body MS ring and regulatory genes, sigma-54 is expressed to act as a flagella specific transcription factor that initiates the transcription of Class III and Class IV genes $(2,50)$. Class III genes encode proteins necessary to complete the basal body and hook structure, which is needed for Class IV flagellin gene expression. Another variation on this theme is the regulation of flagella synthesis in Vibrio cholerae. V. cholerae regulates the synthesis of its flagella by a cascade through four classes of motility genes 
controlled by both sigma-54 and sigma-28 transcriptional activators (42). The Class I product FIrA acts with sigma-54 to transcribe Class II genes, including genes for the MS ring, chemotaxis, and regulatory genes for FliA and FIrC (42). The Class III gene transcription for basal body, hook, and core flagellin is initiated by sigma-54 and its activator FIrC (42). Expression of the Class IV genes is dependent on the sigma-28 transcription factor to complete flagella assembly (42). Similar to E. coli and S. enterica, completion of the basal body-hook structure acts as a general assembly checkpoint in the transcriptional regulation of flagella synthesis for these other bacteria as well.

The regulation of PF synthesis in $B$. burgdorferi differs markedly from that in other species of bacteria. Homologs for the class II transcriptional regulatory genes fliA (sigma-28) and flgM have not been identified, and no sigma-28 promoters are evident in the $B$. burgdorferi genome $(15,35)$. Furthermore, when analyzing promoter sequences in B. burgdorferi, no sigma-54 recognition sequences were detected in motility operons. Instead, this bacterium has sigma70 promoters controlling its motility genes $(19,35)$. Other than the apparent lack of flagella-specific transcriptional regulatory proteins utilized by other bacteria, little is known about the regulation of PF synthesis in B. burgdorferi. Currently, efforts are being made to elucidate the events involved in PF regulation and synthesis. Motaleb et.al. (39) described a null mutant in the major flagellin gene flaB that does not make flagella, is non-motile, and has a rod-shaped morphology. Complementation of this mutant in cis restored its motility and 
wave-like morphology (47). The flaB::kan mutant, MC-1, was characterized in detail by Western blot to assess the effects of this mutation on other motility and chemotaxis genes. Interestingly, the only putative motility and chemotaxis protein altered with respect to accumulation in MC-1 was FlaA, the minor PF filament protein (40). The reason for this inhibition of FlaA is poorly understood, but results revealed that the decrease of FlaA in a flaB mutant occured posttranscriptionally. Specifically, evaluation of MC-1 by real-time RT-PCR revealed that the flaA message was transcribed at wild-type levels, indicating that the reduction of FlaA synthesis does not occur by transcriptional regulation (40).

As described above for other bacteria, the hook is necessary for flagella structure and also provides a regulatory checkpoint during flagella synthesis. We tested these two aspects in regard to the importance of the PF hook protein in $B$. burgdorferi. First, to test the hypothesis that the hook is critical for flagella based motility and the flat-wave morphology, we used targeted mutagenesis to inactivate the gene encoding the PF hook protein by insertion of a kanamycin resistance cassette. Our results further support those obtained with the flaB mutant $\mathrm{MC}-1$, which indicate the importance of PF involvement in motility and morphology (39). Next, to investigate the effects of flgE mutation on the regulation of PF proteins, we used Western blot and quantitative RT-PCR analysis to monitor the expression of other motility and chemotaxis genes. Our results indicate that the inhibition of flagellar hook negatively impacts both FlaA and FlaB filament protein accumulation specifically at a posttranscriptional level. 
Further, we used spectinomycin to assess whether the inhibition of FlaA and FlaB accumulation was the result of protein turnover or translational control. 


\section{Materials and Methods}

Bacterial strains and growth conditions. The high-passage $B$. burgdorferi sensu stricto strain $\mathrm{B} 31 \mathrm{~A}$ was grown at $33^{\circ} \mathrm{C}$ in $\mathrm{BSK}-$ II liquid or on plates in $3 \%$ carbon dioxide $(7,39,46)$. The non-motile flgE::kan mutant (SC-1) and flaB::kan mutant (MC-1) cells were grown on solid or in liquid BSKII with $300 \mu \mathrm{g} / \mathrm{ml}$ of kanamycin. Swarm plate assays were performed on $0.35 \%$ agarose with BSK-II diluted 1:10 as previously described (39). E. coli JM109 cells were grown in Luria Bertani (LB) broth with the appropriate antibiotic.

Construction of the flgE::kan mutant. The flgE hook gene (BB0283) and flanking DNA were amplified by PCR using primers P1 and P2, described in Table 1 (8) (www.tigr.org). The PCR product obtained was cloned into a pGEMT vector (Promega) to form the plasmid pGE. The kanamycin resistance cassette (kan) used to inactivate flgE was described previously (7). The $1.3 \mathrm{~kb}$ kan cassette was engineered with flanking EcoRI sites (P3 and P4, Table 1) and ligated into pGE at the single EcoRI site located within flgE to yield a $6.0 \mathrm{~kb}$ pGEK. Kanamycin-resistant colonies were screened by PCR and by restriction mapping to identify plasmids harboring pGEK with kan transcribed in the same direction as flgE (15). The flgE-kan portion of the construct was PCR amplified with primers $\mathrm{P} 1$ and $\mathrm{P} 2$, and approximately $5 \mu \mathrm{g}$ of the linear DNA was electroporated into competent $B$. burgdorferi B31A according to targeted mutagenesis methods described elsewhere $(39,46)$. After 28 days of incubation, small colonies on kanamycin plates were picked and grown in BSK-II liquid. Cells were viewed under dark field microscopy. 
Electrophoresis and Western blotting. Approximately $5 \mathrm{ml}$ of late logrithmic phase cells were centrifuged at $8000 \mathrm{Xg}$ at $23^{\circ} \mathrm{C}$ for 10 minutes, washed with water, and centrifuged at $13,000 \mathrm{Xg}$ at $23^{\circ} \mathrm{C}$ for 5 minutes. The cell pellets were resuspended in $50 \mu \mathrm{l}$ of sodium dodecyl sulfate (SDS) reducing buffer and boiled for 5 minutes. Total protein from cell lysates was quantified by spectrophotometer using the BIO-RAD protein assay. Equal amounts of total protein from each sample were loaded onto a polyacrylamide gel. SDSpolyacrylamide gel electrophoresis (SDS-PAGE) and Western blotting with the enhanced chemiluminescent detection system $(E C L)$ were performed as previously described (17). Polyclonal Anti-FlaB was generously provided by M. Caimano (University of Connecticut Health Center, Farmington, CT). Polyclonal anti-MotB was given by J. Carroll (University of Pittsburgh, Pittsburgh, PA) and polyclonal E. coli FliM was kindly provided by D. Blair (University of Utah, Salt Lake City, UT). Polyclonal anti-Flil was generated by Y. Ge (16). Polyclonal CheY 3 and CheX antisera were provided by M. Motaleb, and polyclonal CheA2 antiserum was provided by C. Li (West Virginia University, Morgantown, WV). Monoclonal antibody H9724 was kindly donated by A. Barbour (University of California, Irvine, CA). Monoclonal anti-FlaA was generously provided by B. Johnson (Centers for Disease Control and Prevention, Atlanta, GA). Monoclonal anti-DnaK was kindly provided by J. Benach (State University of New York, Stony Brook, NY). 
Production of recombinant FlgE and antisera. The flgE gene was amplified by PCR from B. burgdorferi DNA using primers P9 and P10 (Table 1). The PCR product was cloned into the pCR T7/NT-TOPO expression vector (Invitrogen) which encodes an amino-terminal histidine tag attached to the flgE gene. BL21 Star (DE3) pLysS One Shot chemically competent cells (Invitrogen) were transformed and grown overnight in liquid LB with $100 \mu \mathrm{g} / \mathrm{ml}$ ampicillin and 34 $\mu \mathrm{g} / \mathrm{ml}$ chloramphenicol. Induction with isopropyl- $\beta$-D-thiogalactopyranoside (IPTG) was performed according to the manufacturer's instructions.

Recombinant FlgE was purified by a nickel agarose column and concentrated in $10 \mathrm{kD}$ molecular weight cut off Amicon Ultra centrifugal concentrators (Millipore). Rabbits were initially immunized with $100 \mu \mathrm{g}$ of purified recombinant FlgE protein in Freund's Complete Adjuvant and subsequently boosted twice over the period of 1 month with $50 \mu \mathrm{g}$ of recombinant FlgE in Freund's Incomplete Adjuvant. Blood samples were collected and centrifuged under low speed to separate the serum fraction.

Southern blotting. Genomic DNA was purified from B31A and SC-1 as previously described (3) and resuspended in water and stored overnight at $4^{\circ} \mathrm{C}$. DNA samples were then treated with $\mathrm{RNaseA}$ at $37^{\circ} \mathrm{C}$ for 30 minutes to remove contaminating RNA. The genomic DNA was digested with Hind III at $37^{\circ} \mathrm{C}$ for 3 hours and run on $0.8 \%$ agarose gel at $30 \mathrm{~V}$ for 6 hours. DNA fragments were transferred by capillary action from the agarose gel to a Nylon membrane in $10 \mathrm{X}$ SSC buffer overnight. Probes for the kanamycin cassette and the $1 \mathrm{~kb}$ molecular 
weight ladder were labeled using the DIG DNA Labeling kit, and Southern blotting was performed according to the manufacturer's instructions (Roche).

\section{Quantitative reverse transcriptase polymerase chain reaction (qRT-PCR).}

Total RNA was isolated using the RNeasy kit (Qiagen) was treated with DNase to remove contaminating DNA. RNA concentration was determined at $260 \mathrm{~nm}$, and the RNA purity was determined from the $260 \mathrm{~nm} / 280 \mathrm{~nm}$ ratio (27). Reverse transcription was performed on $100 \mathrm{ng}$ of total RNA using the Reverse Transcription System according to the manufacturer's instructions (Promega). Negative controls were run for each sample by omitting the reverse transcriptase enzyme. Random hexamers (Roche) were used as primers for general cDNA production in all samples (25). The cDNA was amplified (ProofStart DNA Polymerase, Qiagen) using flaA P15 and P16 primers or flaB P17 and P18 primers to generate DNA for use as standards (Table 1). Sample cDNA, standard DNA, and negative control (No RT) were run simultaneously with flaA or flaB primers. Quantitative PCR was performed on the LightCycler (Roche) using the QuantiTect SYBR Green PCR kit (Qiagen). The enolase gene (BB0337), eno, was used with primers $\mathrm{P} 19$ and $\mathrm{P} 20$ as a control in each experiment to normalize sample data (Table 1). The enolase gene has been used previously to normalize quantitative RT-PCR data (28).

Protein Turnover Assay. Wild type and SC-1 cells were enumerated by flow cytometry (4). An equal volume of fresh BSK-II media was added to $1 \times 10^{9}$ wild 
type or SC-1 cells, and spectinomycin was added to a final concentration of 100 $\mu \mathrm{g} / \mathrm{ml}$. The cultures were incubated at $33^{\circ} \mathrm{C}$ for $0,2,4,6$, or 12 hours, at which time the cells were centrifuged at $8,000 \mathrm{Xg}$ at $4^{\circ} \mathrm{C}$ for 15 minutes. The cell pellets were washed in water and centrifuged at $13,000 \mathrm{Xg}$ at $23^{\circ} \mathrm{C}$ for 5 minutes. Washed cell pellets were stored at $-80^{\circ} \mathrm{C}$ overnight. Cell pellets were resuspended in water, then SDS reducing dye was added and the samples were boiled for 5 minutes. Protein concentrations from cell lysates were determined by spectrophotometer using the Bio-Rad protein assay. SDS-PAGE and Western blot were performed as previously described.

Light and Electron Microscopy. Cell morphology and motility of wild type and SC-1 were observed by dark field microscopy. To identify the presence of PFs, wild type and SC-1 were fixed and embedded as described (21), and thin sections were analyzed by electron microscopy using a JEOL JEM 1220 microscope at an accelerating voltage of $80 \mathrm{kV}$. 


\section{Results}

Isolation of flgE::kan mutant. The flagellar hook structure, which is encoded by the flgE gene product, is essential for the assembly and function of flagella in many bacteria, including spirochetes. We hypothesized that flgE mutant in $B$. burgdorferi would be phenotypically similar to the flaB PF deficient mutant MC-1. MC-1 is non-motile, rod-shaped, and lacks the wave-like morphology of the wild type. We also considered that a mutation in flgE would give us insight into the unique regulation of $\mathrm{PF}$ synthesis, as these bacteria lack the sigma factor dependent cascade control of flagellar regulation found in other bacteria. Accordingly, we inactivated the gene encoding flagellar hook protein by targeted mutagenesis. The flgE gene (BB0283) in B. burgdorferi contains the open reading frame of highest homology to the hook in other bacteria, including flgE of E. coli with $62 \%$ similarity. The flgE gene was amplified by PCR using primers P1 and P2, and cloned into pGEM-T (Figure 1, Table 1). A kanamycin resistance cassette fused to the $B$. burgdorferi flgB promoter (kan) with flanking EcoRI sites was inserted at the EcoRI site within the flgE gene to yield plasmid pGEK. The flgE-kan region of $\mathrm{pGEK}$ was amplified by PCR using the primers $\mathrm{P} 1$ and $\mathrm{P} 2$, and the linear DNA product was electroporated into B. burgdorferi as previously described (22). All primer sequences are listed in Table 1. 


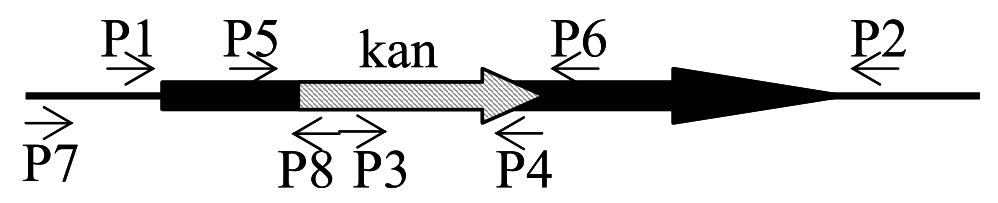

Figure 1. Map of kan insertion into flgE and selected PCR primers.

The flgE gene (large arrow) was amplified using primers P1 and P2, and cloned into pGEM-T Easy Vector to yield pGE. The kan cassette (hashed arrow) was amplified with flanking EcoRI sites using primers P3 and P4, and was inserted into flgE at its single EcoRI site to yield pGEK. The linear DNA fragment containing the flgE-kan construct was used for electroporation. All PCR primers are denoted by small arrows. 
Whereas wild type colonies appear approximately at day 10 of incubation, several small surface colonies appeared at day 28 after electroporation and plating on kanamycin selection. Six colonies of the same rod-shaped morphology were grown in liquid medium with kanamycin. Two of these colonies, SC-1 and SC-2, were examined by PCR and contained kan within the flgE gene. Subsequently, SC-1 was recloned and analyzed in greater detail.

The SC-1 mutant cells were genetically characterized by Southern blot and PCR analyses. To determine if targeted mutagenesis occurred with the flgE::kan construct as expected, genomic DNA from wild-type and SC-1 cells were digested with HindllI. The predicted size of the flgE::kan Hindlll fragment is $3.8 \mathrm{~kb}$. The kan gene obtained by PCR amplification using primers P3 and P4 (Table 1, Figure 1) was used as a probe for Southern analysis. The labaled kan hybridized to one unique Hindlll fragment of predicted $3.8 \mathrm{~kb}$ size (Figure 2). These results suggest that kan was inserted only once in the genome at the predicted location. PCR analysis also demonstrated that kan was inserted at the correct location within flgE in SC-1. Using primers P5 and P6 within flgE that flank the kan insertion site (Figure 1), we obtained a predicted $1.8 \mathrm{~kb}$ product in SC- 1 cells (Figure 3 ) and SC-2 cells (data not shown). The absence of a $0.5 \mathrm{~kb}$ product, indicative of the wild type flgE gene, confirmed the insertion of our construct at the appropriate location in SC-1 and SC-2. Furthermore, an analysis of PCR products using the primers P8 within kan and the external primer P7, verified that $f l g E$ was interrupted by kan in SC-1. These results also indicate that 
kan was transcribed in the same direction as flgE in SC-1 (Figures 1, 3). Taken together, results from Figure 2 and Figure 3 indicate that kan inserted within flgE at the desired location, and there were no additional insertions of the construct DNA elsewhere in the recipient bacterial genome.

We directly looked for the presence of FlgE protein in SC-1. To test for the hook protein, we raised polyclonal antisera to recombinant $B$. burgdorferi FlgE, and analyzed cell lysates by Western blotting. We found a strong reactive band at $46 \mathrm{kDa}$, which is in accordance with the predicted molecular weight of FlgE in B. burgdorferi. No $46 \mathrm{kDa}$ band was detected in SC-1. These results indicate that SC-1 was deficient in FlgE synthesis or accumulation (Figure 4). 


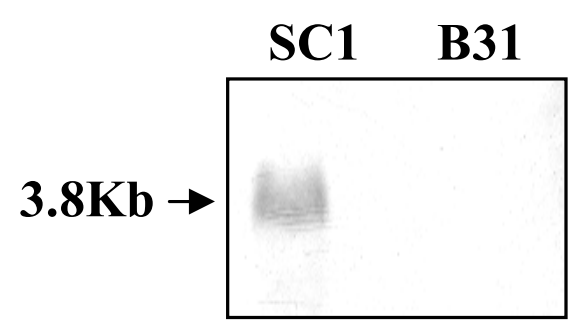

Figure 2. Southern blot analysis of SC-1 and wild type B31.

Genomic DNA from wild type and SC-1 digested with HindIII was used for electrophoresis. The kan cassette was amplified using primers $\mathrm{P} 3$ and $\mathrm{P} 4$, and used as the DIG-labeled probe.

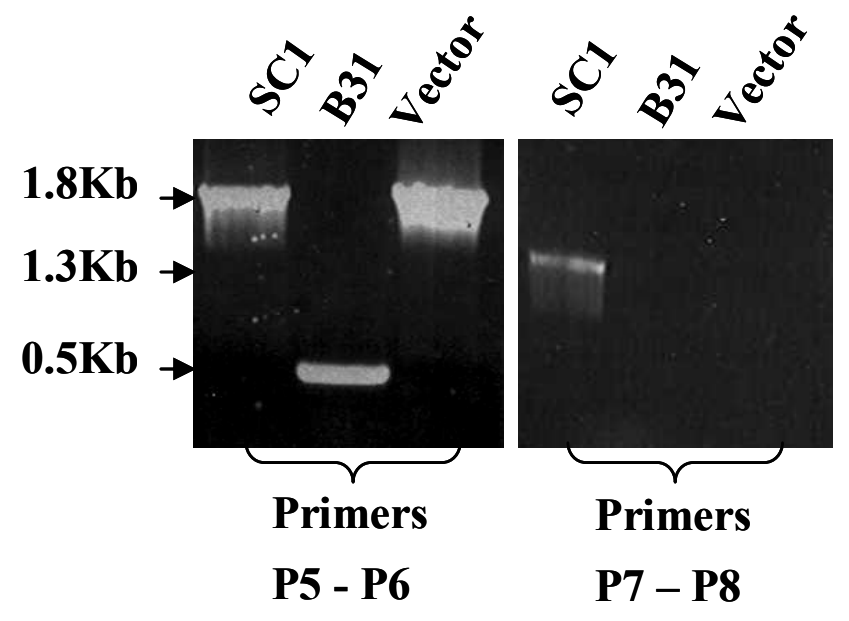

Figure 3. PCR analysis of SC-1.

PCR analysis of SC-1, wild-type, and pGEK using primers P5 and P6 within flgE (lanes 1-3) or primers from sequences outside of flgE and within kan, P7 and P8 (lanes 4-6). The original flgE-kan construct on the plasmid pGEK was used as a vector control. 


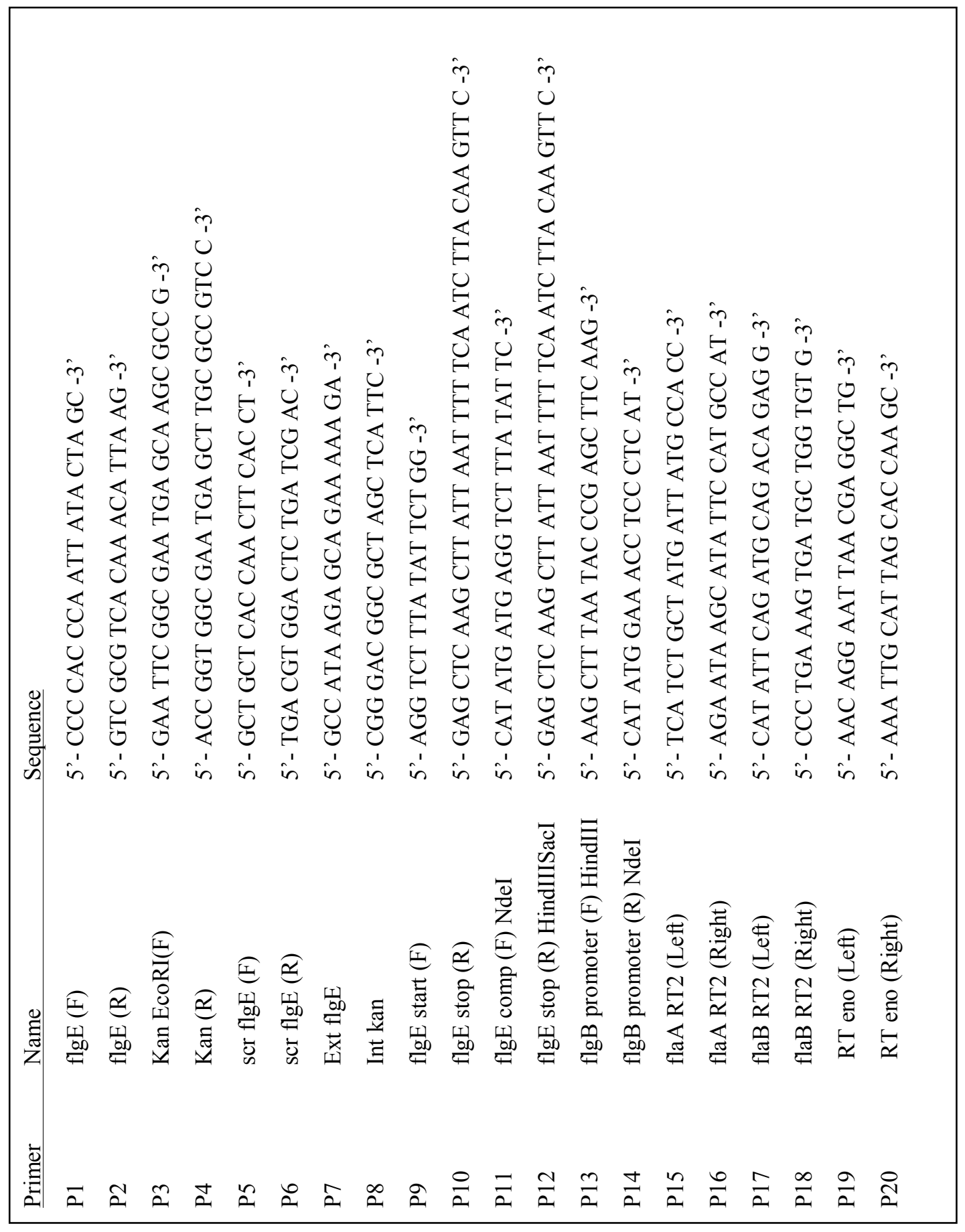

Table 1. Primer sequences. 
Wild type $\quad$ SC-1

\section{FliI}
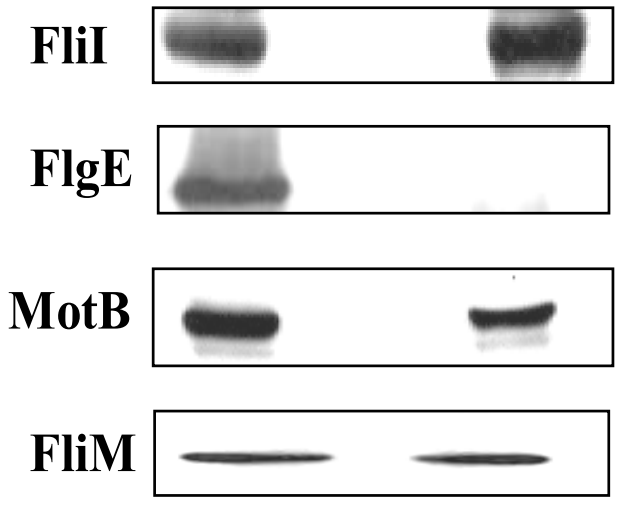

Figure 4. Western blot analysis of gene products from the flgB operon.

Equal amounts of cell lysate from wild type (B31) and SC-1 were separated by SDS-PAGE. Western blotting was carried out using the indicated antisera. Western blot with anti-DnaK was used as a control for all blots (data not shown). The flil gene is located upstream of the flgE gene, whereas motB and fliM genes are located downstream of the flgE gene. 
Altered motility and morphology of SC-1. Previous studies of a B. burgdorferi flaB::kan mutant MC-1 indicated that a loss in PFs resulted in a non-motile phenotype and a striking alteration in cell morphology; the flaB mutant cells were rod-shaped instead of wave-like. Those findings suggest that the PFs are necessary for motility and the wave-like morphology characteristic of wild type cells (Figure 5). Because the hook protein is essential for flagellar morphogenesis in other bacteria, we hypothesized that SC-1 would have a similar phenotype. Darkfield examination of SC-1 indicated that the cells were completely non-motile. Furthermore, SC-1 failed to spread on swarm agar plates. Specifically, wild type cells migrated away from the site of inoculation and formed a ring $22 \mathrm{~mm}$ at day 5; no swarming was evident around the $9 \mathrm{~mm}$ site of inoculation for SC-1 (Figure 5). Similar results of $9 \mathrm{~mm}$ were found with the nonmotile mutant MC-1. These results taken together indicate that SC-1 was nonmotile.

The morphology of mutant SC-1 was analyzed in detail. Darkfield microscopy revealed that the cells were rod-shaped in a manner similar to the mutant MC-1 (Figure 4). Whereas 7-11 PFs were evident in wild-type cells, electron microscopic examination of thin sections of SC-1 revealed that these cells completely lacked PFs. Similar results have been reported for the flaB::kan mutant, MC-1 (39). These results further support the conclusions from MC-1 that the PFs influence the shape of the cells and have a pronounced skeletal function. 

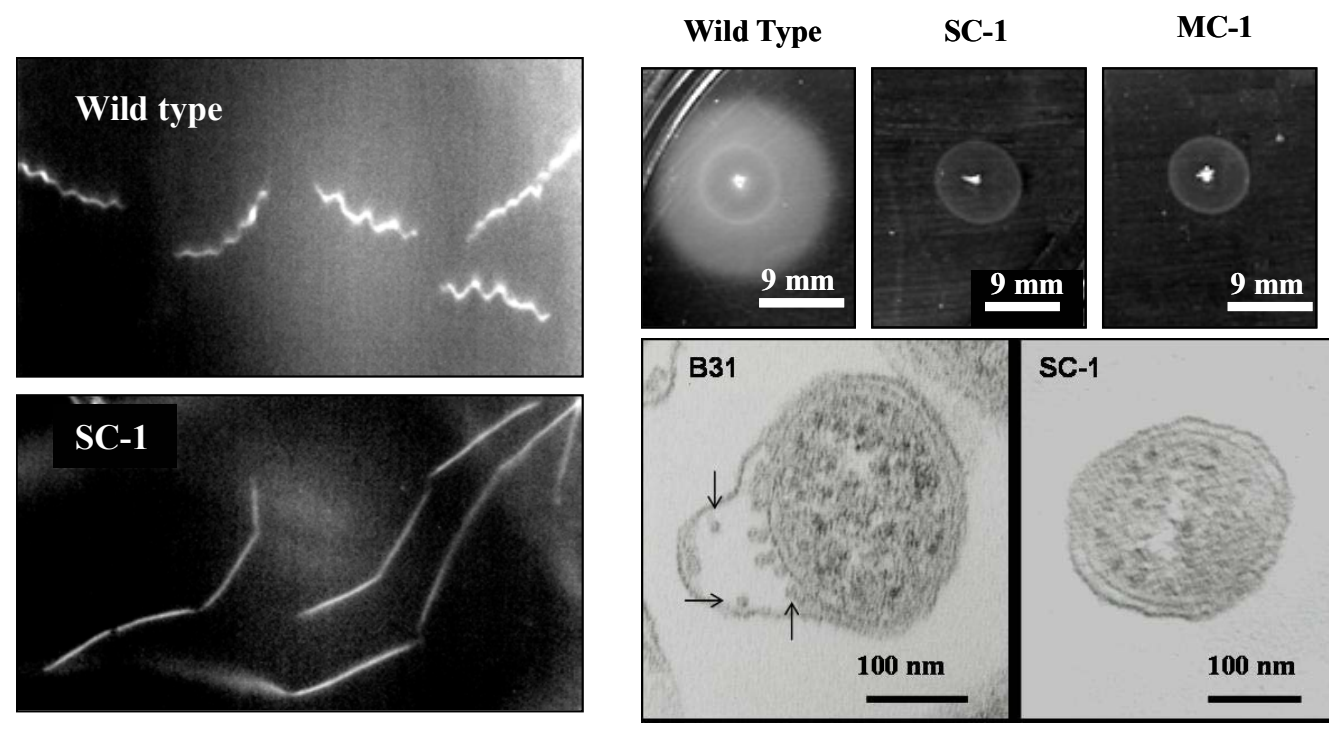

\section{Figure 5. Cell motility and morphology in SC-1.}

Wild type (B31A) cells viewed under dark field microscopy have a wavy morphology. SC-1 cells are rod-shaped. Wild type (B31) and SC-1 were plated on soft agar for 5 days. A white swarm ring $(22 \mathrm{~mm})$ expanding from the site of inoculation ( $9 \mathrm{~mm}$ for each sample) indicates motility in wild type cells. The previously described non-motile flaB::kan mutant MC-1 was used as a negative control. Thin sections examined by electron microscopy show PFs, denoted by arrows, in B31. No PFs are seen in the periplasmic space in SC-1. 
Motility gene expression in the flgB operon. flgE maps near the center of the large $f \lg B$ motility operon in the $B$. burgdorferi chromosome. This operon is $21 \mathrm{~kb}$ long, and consists of 26 putative open reading frames. To determine the effect on the expression of genes both upstream and downstream of flgE, we carried out Western blot analysis on select genes of this operon. A protein involved in flagella assembly, Flil, is encoded by flil located five genes upstream of flgE. Flil was found to accumulate in amounts similar to the wild-type in SC-1 (Figure 4). Similarly, Western blot analysis of MotB consistently indicated that this protein was slightly reduced compared to the wild-type. The motB gene is three open reading frames downstream of $f l g E$ and encodes a flagellar motor protein. In addition, accumulation of the basal body protein FliM in SC-1 was equivalent to the wild-type; fliM is located five open reading frames downstream of flgE (Figure 4). Taken together, Western blot analysis indicated that the insertion of kan into flgE did not appreciably alter the expression of the other select motility genes both upstream and downstream of the mutation.

Decrease in periplasmic flagellar filament proteins. In some bacteria, mutants that fail to synthesize the flagellar hook do not synthesize or accumulate flagellar filament proteins $(1,2,11)$. Accordingly, we used Western blot analysis to assay for the accumulation of the major FlaB PF filament protein of $41 \mathrm{kDa}$, and the minor FlaA filament proteins of $37 \mathrm{kDa}$ and $38 \mathrm{kDa}$ in SC-1 (Figure 6). Using densitometry with DnaK as an internal control, we detected a marked reduction of FlaB accumulation in SC-1 cells relative to the wild-type. We find 
that FlaB accumulation is only $18-30 \%$ wild type levels. With respect to FlaA, this protein has previously been shown to yield two bands on Western blotting: a major band at $38 \mathrm{kDa}$, and a minor band at $37 \mathrm{kDa}(40)$. While we were unable to detect the $37 \mathrm{kDa}$ protein band, we found a greater than $85 \%$ reduction in the 38 $\mathrm{kDa}$ band of FlaA in SC-1. The reduction of FlaA in SC-1 exceeded that previously observed in MC-1 (lane 3). Furthermore, similar results demonstrated a marked decrease of FlaA and FlaB in SC-2 (data not shown). These results indicate that although the flgE::kan mutation markedly inhibited FlaA and FlaB accumulation, it did so incompletely because small amounts of these proteins are still detected.

Expression of chemotaxis genes in the flaA operon. The flagellin gene fla $B$ is monocistronically transcribed. However, the gene encoding FlaA is followed by downstream chemotaxis genes including che $A 2$, cheW 3 , cheX, and cheY3 that comprise the flaA operon $(16,35)$. We used Western blotting to determine whether protein products from genes downstream of flaA were also expressed at reduced levels. In contrast to FlaA expression, blots probed with antisera to CheA2, CheW3, CheX, and CheY3 revealed that these proteins accumulated at wild-type levels in SC-1 cells (Figure 7). With unaltered expression of proteins encoded downstream in the flaA operon, the results strongly suggest that the mutation in flgE specifically affects the synthesis or accumulation of the PF filament protein FlaA. Similar results have been reported for the decrease of FlaA accumulation in MC-1 (40). 

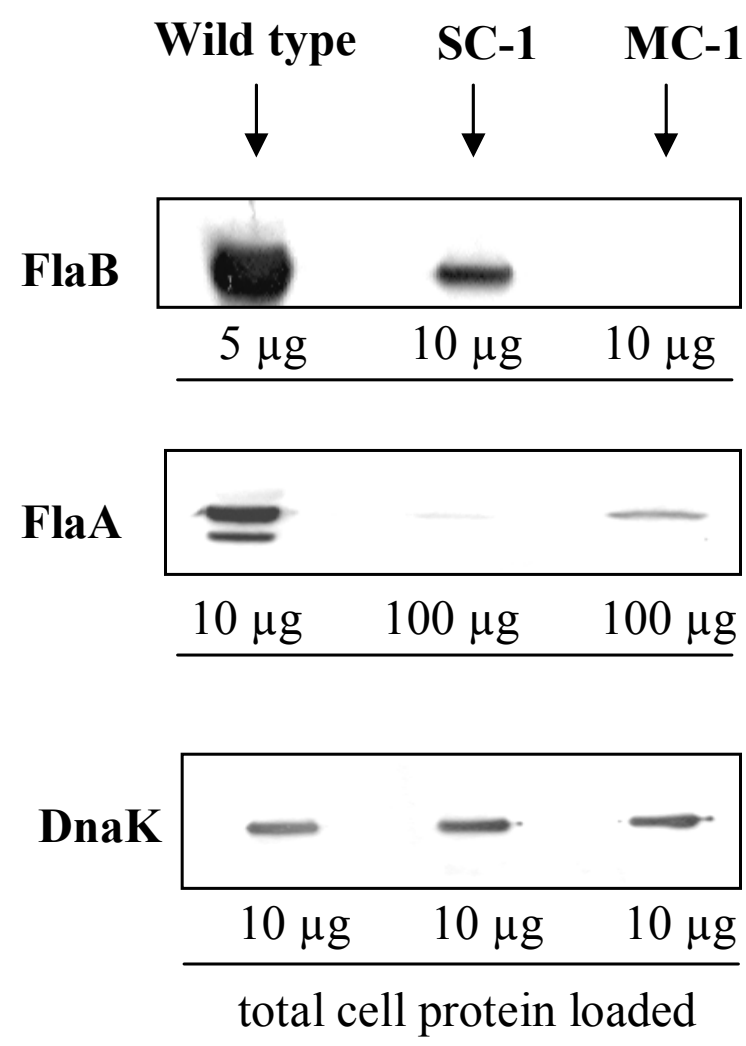

Figure 6. Wesern blot analysis of PF filament proteins.

Cell lysate from wild type (B31A), SC-1, and MC-1 (flaB::kan) were separated by SDS-PAGE. Western blotting was carried out using polyclonal anti-FlaB or monoclonal anti-FlaA. Western blot with anti-DnaK was used as a control. The amount of total cell lysate loaded in each well is indicated. 


\section{flaA CheA2 CheW3 CheX CheY3}

\section{Wild type $\quad$ SC-1}

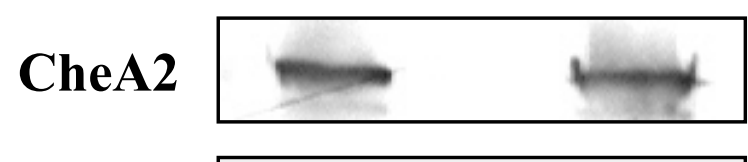

CheW3

CheX

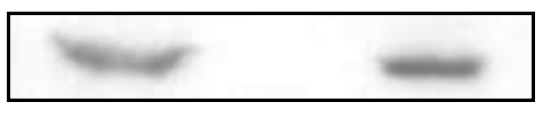

CheY3

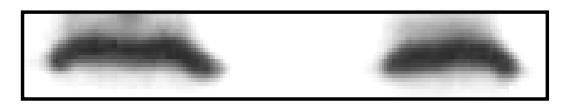

Figure 7. Western blot analysis of gene products downstream of flaA.

Equal amounts of cell lysate from wild type and SC-1 were separated by SDSPAGE. Western blotting was carried out using the indicated antisera. Western blot with anti-DnaK was used as a control for these blots (data not shown). The location of genes in the flaA operon is shown at the top of the figure. 
Decrease in FlaA and FlaB is unrelated to mRNA synthesis. Although flagellin synthesis is regulated at the transcriptional level in other bacteria, the regulation of PF gene expression is poorly understood in $B$. burgdorferi. We used real-time quantitative RT-PCR to determine if the reduction of the FlaB and FlaA in SC-1 was the result of transcriptional regulation. We found that the levels of fla $A$ and flaB messages in SC-1 were the same as found for the wild-type (Figure 8). These results indicate that the reduction of FlaB and FlaA accumulation does not result from a decrease in mRNA, or regulation at the transcriptional level, in the flgE mutant of $B$. burgdorferi.

PF Filament Protein Turnover in SC-1. The quantitative RT-PCR results indicate that the lack of accumulation of FlaA and FlaB in SC-1 occurs posttranscriptionally. The lack of accumulation could result from protein turnover, as reported for FlaA in MC-1 (40). To test for protein turnover, spectinomycin was added to growing cells of SC-1 to arrest translation (40), and the levels of FlaB and FlaA were assayed over time. The amount of FlaA accumulation in SC-1 decreased after the addition of spectinomycin with an estimated half-life of 2 hours; only a trace amount of FlaA was detected at 12 hours (Figure 9). No decrease in FlaA accumulation occurred in wild-type cells. A similar decrease in the accumulation in FlaA in MC-1 has been previously reported (40). These results indicate that the decrease in FlaA accumulation in SC-1 could be explained, in part, by protein degradation. In contrast to FlaA, FlaB levels 
remained stable over 12 hours in both wild type and SC-1 cells. Because there is no detected degradation of FlaB in SC-1, translational control is evidently involved in the decrease of FlaB accumulation. These results suggest that the hook may play a key role in translational control of FlaB. 

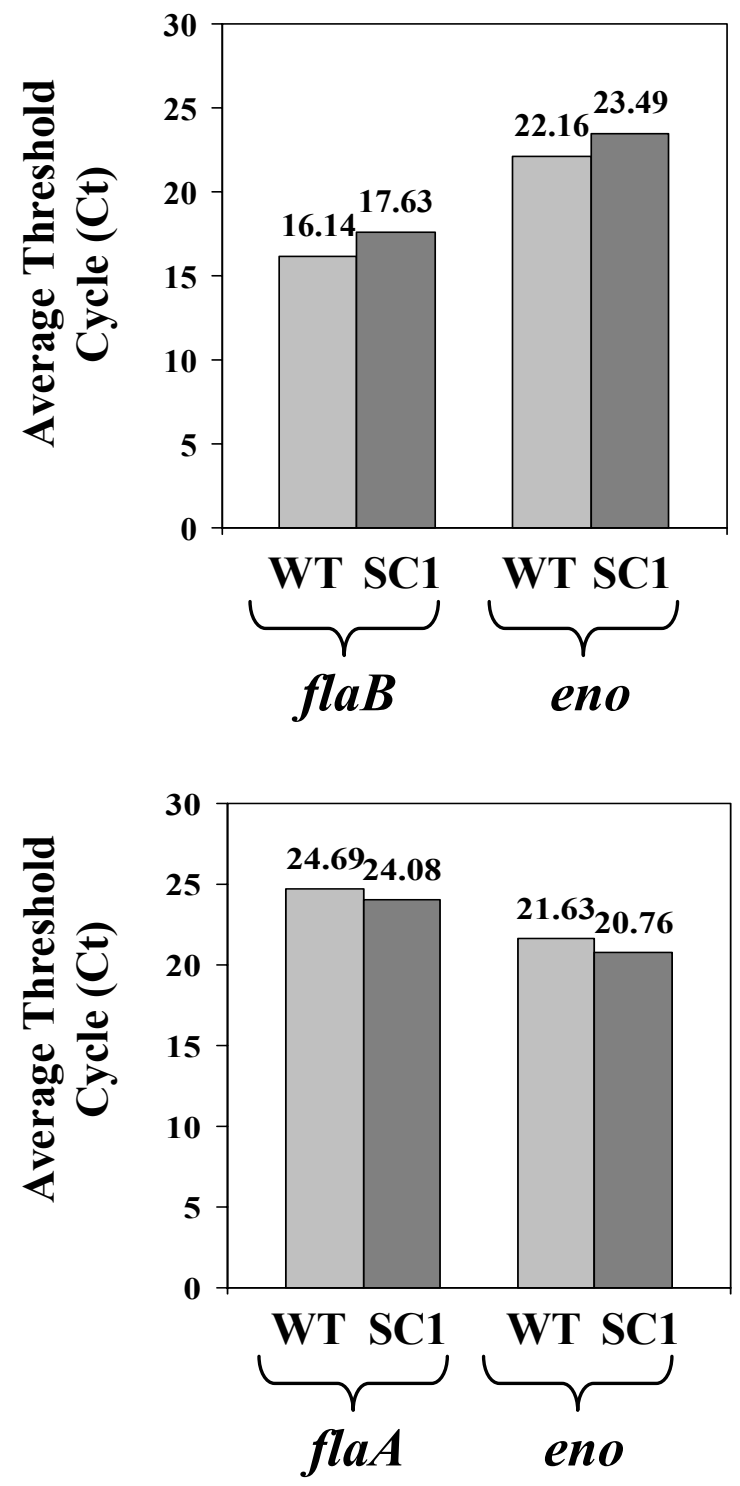

Figure 8. Quantitative RT-PCR of filament genes.

Reverse transcription with random hexamer primers generated cDNA using total RNA from wild type and SC-1. Signal from real-time PCR using specific primers for flaA or flaB was quantified with SYBR Green fluorescent dye on a LightCycler. The data from duplicate samples are expressed as the average threashold cycle $(\mathrm{Ct})$, or the PCR cycle at which the sample signal exceeds the background fluorescence. The enolase gene, eno, was used as a control in each experiment. 


\section{WT}
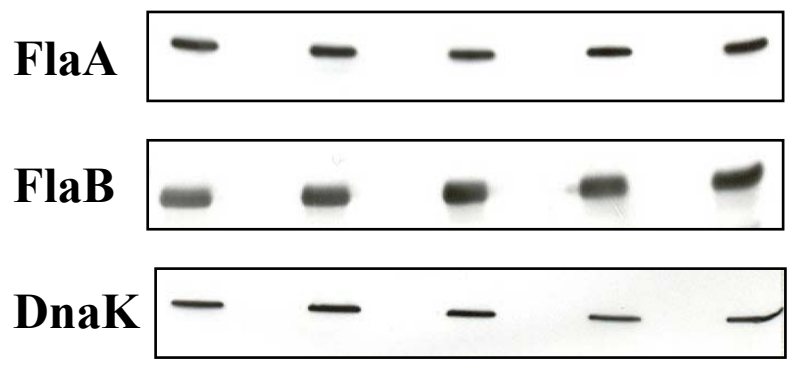

\begin{tabular}{rllll} 
Time: 0 & 2 & 4 & 6 & 12 \\
\hline $\mathrm{hr}$ & $\mathrm{hr}$ & $\mathrm{hr}$ & $\mathrm{hr}$ & $\mathrm{hr}$
\end{tabular}

\section{SC1}

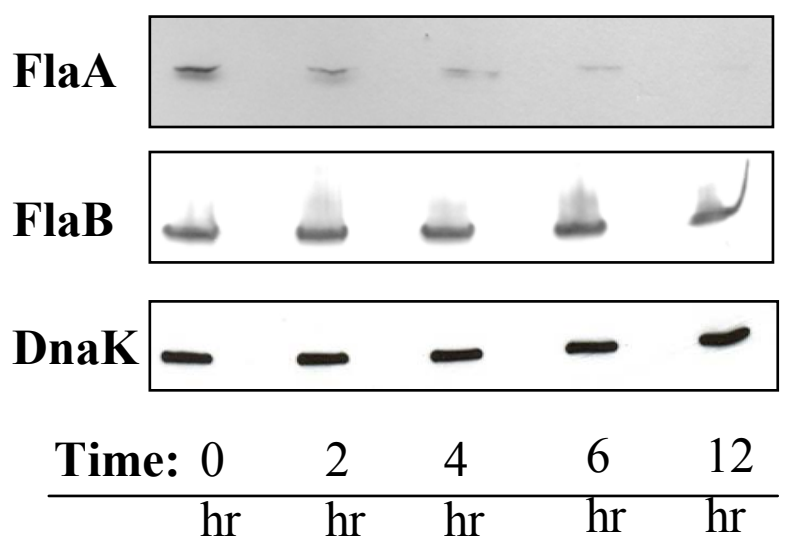

Figure 9. Protein degradation assay.

Spectinomycin $(100 \mu \mathrm{g} / \mathrm{ml})$ was added to $1 \times 10^{9}$ wild type or SC-1 cells to arrest translation. Samples were collected at the indicated time points. All wild type blots contained $5 \mu \mathrm{g}$ of cell lysate. The SC-1 blots contained $100 \mu \mathrm{g}$ of cell lysate for the anti-FlaA blot, $50 \mu \mathrm{g}$ of cell lysate for the anti-FlaB blot, and $5 \mu \mathrm{g}$ of cell lysate for the anti-DnaK blot. 


\section{Discussion}

The flagellar hook acts as a universal joint to fulfill a structural role in the function and assembly of flagella. In addition, the hook also plays a role in assembly by acting as a general checkpoint for the regulation of flagella synthesis in many bacteria. However, the regulation of PF synthesis in $B$. burgdorferi differs from that of other bacteria and is not well understood. To begin understanding the mechanism behind PF synthesis and its regulation, we targeted the PF hook gene. In B. burgdorferi, the hook gene is located on the chromosome at position 294780 to 293452 , which falls in the middle of the large flgB operon spanning 26 genes over $21 \mathrm{~kb}$ (16) (www.TIGR.org). The hook gene, identified as flgE in $B$. burgdorferi, shares high similarity to hook genes in other bacteria including the following: Escherichia coli (62\%), Treponema denticola (59\%), Caulobacter crescentus (56\%), Campylobacter jejuni (50\%), and Helicobacter pylori (48\%). Inactivation of flgE in these bacteria results in non-motility and the inability to complete flagella synthesis $(1,2,11,31,38)$.

The PF hook is essential for motility and wave-like morphology in $B$. burgdorferi. We found that inactivation of flgE with a kanamycin resistance cassette resulted in non-motile, rod-shaped cells, which is consistent with observations of flagella-less $B$. burgdorferi $(22,39,40,45,47)$. Null mutants in flaB were previously characterized and found to be rod-shaped, non-motile, and lack PFs $(39,47)$. Using various methods, we determined that the flgE::kan construct recombined at only one location in the SC-1 genome. Therefore, the 
phenotype observed in SC-1 can be attributed to the inactivation of flgE in SC-1, rather than aberrant insertion of the construct elsewhere in the genome. As flgE is located within the large flgB operon and transcribed with other genes (20), we were concerned that polar effects from inactivating flgE may contribute to the observed phenotype. We analyzed SC-1 by Western blotting and found that two genes downstream of $f l g E$ were expressed at near wild type levels. It is unknown whether those genes downstream of flgE in SC-1 are transcribed from the native $f l g B$ operon promoter or from the $f l g B$ promoter driving transcription of the kan cassette.

Motility mutants of $B$. burgdorferi have proven to be a challenge for complementation. However, functional complementation of SC-1 would show definitively that the observed phenotype is due solely to flgE inactivation, because genes downstream of flgE are expressed near wild type levels in SC-1. Based on a comparison of data from MC-1, we are confident that the observed phenotype in SC-1 is due solely to the inactivation of flgE and the resultant loss of PFs. Similar to SC-1, targeted mutagenesis of flaB resulted in rod-shaped MC-1 that lacked motility, lacked PFs, and accumulated reduced amounts of FlaA compared to wild type cells $(39,40)$. Although, in previous work, MC-1 was able to support a shuttle vector on double antibiotic selection, most of the resultant clones were not functionally complemented (47). Functional complementation of MC-1 was only accomplished in cis, where recombination occurred between the shuttle vector and a flaB region downstream of kan (47). 
In the complemented strain, cells regained PFs, motility, and the wave-like morphology characteristic of wild type cells $(40,47)$. There are no other reports of motility gene complementation from a shuttle vector in B. burgdorferi.

The complementation of flgE in SC-1 and concurrent restoration of wild type motility, morphology, and PFs would definitively demonstrate the importance of the PF hook in B. burgdorferi. Thus far, we have been unable to successfully complement SC-1 despite the use of two different shuttle vectors, pKFSS1 and pBSV6, various methods for preparing competent SC-1 cells, and different cell plating techniques $(13,14,46)$. More specifically, the entire flgE gene was cloned into a pGEM vector directly following the $f \lg B$ promoter sequence. The flgBp-flgE fragment was inserted into both pKFSS1 and pBSV6 shuttle vectors. Two methods were used to introduce these shuttle vectors into SC-1 (14). Electroporation of the construct into SC-1 was attempted initially, and after cells recovered, they were incubated on agar P-BSK media with the appropriate selective antibiotic as previously described (46). Next, we prepared competent SC-1 cells and used the TSS chemical transformation method (13). Neither method for competent cell preparation and transformation yielded positive results. In addition to plating on solid P-BSK, we attempted to culture transformant cells by limiting dilution with liquid BSK in 96 well plates after electroporation or chemical transformation. Again, this alternative plating method did not yield positive results. Despite using different shuttle vectors and cell preparation methods, multiple attempts to achieve functional complementation 
have been unsuccessful in SC-1. Further attempts to complement SC-1 are ongoing.

In contrast to the regulation of flagella synthesis described in other bacteria, our data suggest that the PF hook is not a transcriptional regulatory checkpoint for B. burgdorferi. Flagella synthesis in many bacteria progresses as a cascade of transcriptional events that allow for the ordered expression and assembly of flagellar components. As flagella production is energetically costly to a cell, bacteria utilize regulatory checkpoints to monitor the progress of flagella synthesis. One such regulatory checkpoint is the completion of the basal bodyhook structure, and the concurrent inhibition of flagellin gene transcription by intracellular pools of FlgM binding to sigma-28 $(1,11,11)$. In these bacteria, hook mutants inhibit FlgM from leaving the cell and thus prevent sigma-28 from transcribing flagellin and other late genes $(26,29,34)$. In B. burgdorferi, flgM and fliA have not been identified, and the motility operons are under the control of sigma-70-like promoters rather than flagella-specific sigma-28 promoters (15, $16,18-20,35)$. In this work, we found that SC-1 makes wild type quantities of flaB and flaA mRNA, but only minimal amounts of these two flagellin proteins. Therefore, the negative impact on the synthesis of flagellin proteins in SC-1 does not result from transcriptional regulation during basal body-hook construction.

Bacteria including Caulobacter crescentus, Campylobacter coli, and Helicobacter pylori have external flagella filaments composed of multiple 
flagellins $(12,24,31,33)$. The intact basal body-hook structure is a common assembly checkpoint in these bacteria, as well as in enteric bacteria, yet the outcome of flgE mutation varies depending on species. Different patterns emerge in the assembly of flagellins in these bacteria with hook mutations. The flgE mutants in C. coli and H. pylori are non-motile and lack flagella, but express wild type levels of flagellin that accumulate in the cytoplasm because they cannot be exported and assembled onto an intact hook $(31,38,41)$. Our results from SC-1 are not consistent with the accumulation of flagellin observed in these two species of bacteria. In contrast to these species, C. crescentus messages are transcribed for both flagellins from different types of promoters, but the respective protein production is apparently inhibited post-transcriptionally in the absence of the hook (2). Although $B$. burgdorferi flagellin genes utilize sigma-70 promoters, our results from SC-1 resemble $C$. crescentus somewhat with regard to posttranscriptional inhibition of flagellin accumulation. Our findings are also in agreement with the flgE mutant of $T$. denticola that accumulated barely detectable levels of FlaA and FlaB relative to the wild type (44). Despite transcribing flaB and fla $A$ at wild type levels, SC-1 produces both flagellins at significantly reduced levels compared to wild type proteins. Accordingly, posttranscriptional effects are likely involved in the greatly reduced flagellin levels in SC-1.

Our results demonstrate that accumulation of the flagellin proteins FlaA and FlaB is specifically affected in SC-1. The flaB gene is monocistronically 
transcribed and maps $550 \mathrm{~kb}$ away from flaA. The flaA gene is the first of five genes in a motility and chemotaxis operon. We found by Western blotting that the downstream chemotaxis genes che $A 2$, cheW 3 , cheX, and cheY3 are fully expressed in SC-1. Therefore, the post-transcriptional inhibition of FlaA accumulation is specific with regard to that operon in SC-1. Furthermore, it is noteworthy that the chemotaxis genes downstream of fla $A$ are expressed. In bacteria with cascade control of flagella synthesis, chemotaxis genes are often classified as late genes, and intact FlgE is necessary for their production during the final stages of flagella synthesis. However, we find that chemotaxis proteins encoded in the fla $A$ operon are present at normal levels in the absence of the hook in SC-1. Our results are in agreement with those from MC-1 where FlaA was specifically inhibited in a flaB::kan mutant, while downstream chemotaxis genes cheA2 and cheY3 were expressed at wild type levels (40). The expression of chemotaxis genes in SC-1 lends further support to our conclusions that FlaA and FlaB are specifically inhibited in a SC-1.

We have found that the absence of PF hook impacts flagellin accumulation specifically in $B$. burgdorferi, although not at the transcriptional level. We sought to determine the contribution of protein turnover to the greatly reduced flagellin levels in SC-1. We found that the amount of FlaA in SC-1 diminished over 12 hours to barely detectable levels in the presence of spectinomycin, whereas wild type cells demonstrated FlaA stability under the same conditions. Due to the extremely low levels of FlaA, the lower $37 \mathrm{kDa} F \mathrm{FaA}$ 
band commonly seen in the wild type was not detected in SC-1. One explanation of the FlaA degradation may be the interaction of FlaA with FlaB in the periplasmic space. FlaB is thought to be exported to the periplasmic space through the type III secretion system, whereas FlaA is secreted by a secdependent pathway $(9,32)$. In the absence of hook, FlaB may remain unassembled and protected from proteolysis in the cytoplasm. Conversely, FlaA transported to the periplasmic space could be degraded by proteases because it is unable to associate with FlaB. At this time, we cannot be certain if or how the small amount of FlaA in SC-1 is actually transported to the periplasmic space. Although the FlaA detected in $\mathrm{SC}-1$ is less than that in $\mathrm{MC}-1$, our results support previous findings of FlaA turnover in a flaB mutant (40). Furthermore, because of the difference in FlaA accumulation between MC-1 and SC-1, it is possible that another level of post-transcriptional regulation, in addition to protein degradation, is acting to keep FlaA levels reduced in SC-1.

Unlike FlaA, the amount of FlaB in SC-1 remained constant over 12 hours in spectinomycin. Because these data indicate that FlaB is not degraded in SC1 , our results suggest that protein turnover does not contribute to the reduced levels of FlaB in SC-1. Based on these findings, we must conclude that FlaB synthesis in B. burgdorferi is post-transcriptionally controlled, perhaps at the level of translation. 


\section{Acknowledgements}

We would like to thank Diane Berry for thin section preparations and guidance with electron microscopy. We appreciate critical comments from Chunhao Li.

This research was supported by U.S. Public Health grant AI29743 to N.W.C. and by American Heart Association grant 0365225B to M.A.M. 


\section{References}

1. Aldridge, P. and K. T. Hughes. 2002. Regulation of flagellar assembly. Curr. Opin. Microbiol. 5:160-5.

2. Anderson, D. K. and A. Newton. 1997. Posttranscriptional regulation of Caulobacter flagellin genes by a late flagellum assembly checkpoint. J. Bacteriol. 179:2281-2288.

3. Ausubel, F. M. 1994. Current protocols in molecular biology, p. 4 v. (loose-leaf). John Wiley \& Sons, New York.

4. Bakker, R. G. 2004. Measuring chemotaxis in Borrelia burgdorferi, the Lyme disease spirochete. Dissertation.

5. Barbour, A. G. and S. F. Hayes. 1986. Biology of Borrelia species. Microbiol. Rev. 50:381-400.

6. Berg, H. C. and L. Turner. 1979. Movement of microorganisms in viscous environments. Nature 278:349-51.

7. Bono, J. L., A. F. Elias, J. J. Kupko, B. Stevenson, K. Tilly, and P. Rosa. 2000. Efficient targeted mutagenesis in Borrelia burgdorferi. J. Bacteriol. 182:2445-52.

8. Casjens, S., N. Palmer, R. van Vugt, W. M. Huang, B. Stevenson, P. Rosa, R. Lathigra, G. Sutton, J. Peterson, R. J. Dodson, D. Haft, E. Hickey, M. Gwinn, O. White, and C. M. Fraser. 2000. A bacterial genome in flux: the twelve linear and nine circular extrachromosomal DNAs in an infectious isolate of the Lyme disease spirochete Borrelia burgdorferi. Mol. Microbiol. 35:490-516.

9. Charon, N. W. and S. F. Goldstein. 2002. Genetics of motility and chemotaxis of a facinating group of bacteria: The Spirochetes. Annu. Rev. Genet. 36:47-73.

10. Charon, N. W., S. F. Goldstein, S. M. Block, K. Curci, J. D. Ruby, J. A. Kreiling, and R. J. Limberger. 1992. Morphology and dynamics of protruding spirochete periplasmic flagella. J. Bacteriol. 174:832-40.

11. Chilcott, G. S. and K. T. Hughes. 2000. Coupling of flagellar gene expression to flagellar assembly in Salmonella enterica serovar typhimurium and Escherichia coli. Microbiol. Mol. Biol. Rev. 64:694-708. 
12. Driks, A., R. Bryan, L. Shapiro, and D. J. DeRosier. 1989. The organization of the Caulobacter crescentus flagellar filament. J. Mol. Biol. 206:627-636.

13. Elias, A. F., P. E. Stewart, D. Grimm, M. J. Caimano, C. H. Eggers, K. Tilly, J. L. Bono, D. R. Akins, J. D. Radolf, T. G. Schwan, and P. Rosa. 2002. Clonal polymorphism of Borrelia burgdorferi strain B31 Ml: implications for mutagenesis in an infectious strain background. Infect. Immun. 70:2139-50.

14. Frank, K. L., S. F. Bundle, M. E. Kresge, C. H. Eggers, and D. S. Samuels. 2003. aadA confers streptomycin resistance in Borrelia burgdorferi. J. Bacteriol. 185:6723-7.

15. Fraser, C. M., S. Casjens, W. M. Huang, G. G. Sutton, R. Clayton, R. Lathigra, O. White, K. A. Ketchum, R. Dodson, E. K. Hickey, M. Gwinn, B. Dougherty, J. F. Tomb, R. D. Fleischmann, D. Richardson, J. Peterson, A. R. Kerlavage, J. Quackenbush, S. Salzberg, M. Hanson, R. van Vugt, N. Palmer, M. D. Adams, J. Gocayne, J. C. Venter, and et al. 1997. Genomic sequence of a Lyme disease spirochaete, Borrelia burgdorferi. Nature 390:580-6.

16. Ge, Y. and N. W. Charon. 1997. Molecular characterization of a flagellar/chemotaxis opernon in the spriochete Borrelia burgdorferi. FEMS Microbiol. Lett. 152:425-431.

17. Ge, Y., C. Li, L. Corum, C. A. Slaughter, and N. W. Charon. 1998. Structure and expression of the FlaA periplasmic flagellar protein of Borrelia burgdorferi. J. Bacteriol. 180:2418-25.

18. Ge, Y., I. G. Old, I. S. Girons, and N. W. Charon. 1997. The flgK motility operon of Borrelia burgdorferi is initiated by a sigma 70-like promoter. Microbiology 143 ( Pt 5):1681-1690.

19. Ge, Y., I. G. Old, I. Saint Girons, and N. W. Charon. 1997. Molecular characterization of a large Borrelia burgdorferi motility operon which is initiated by a consensus sigma70 promoter. J. Bacteriol. 179:2289-99.

20. Ge, Y. and N. W. Charon. 1997. Identification of a large motility operon in Borrelia burgdorferi by semi-random PCR chromosome walking. Gene 189:195-201.

21. Goldstein, S. F., K. F. Buttle, and N. W. Charon. 1996. Structural analysis of the Leptospiraceae and Borrelia burgdorferi by high-voltage electron microscopy. J. Bacteriol. 178:6539-45. 
22. Goldstein, S. F., N. W. Charon, and J. A. Kreiling. 1994. Borrelia burgdorferi swims with a planar waveform similar to that of eukaryotic flagella. Proc. Natl. Acad. Sci. USA 91:3433-7.

23. Greenberg, E. P. and E. Canale-Parola. 1977. Relationship between cell coiling and motility of spirochetes in viscous environments. J. Bacteriol. 131:960-9.

24. Guerry, P., R. A. Alm, M. E. Power, S. M. Logan, and T. J. Trust. 1991. Role of two flagellin genes in Campylobacter motility. J. Bacteriol. 173:4757-4764.

25. Hefty, P. S., S. E. Jolliff, M. J. Caimano, S. K. Wikel, J. D. Radolf, and D. R. Akins. 2001. Regulation of OspE-related, OspF-related, and Elp lipoproteins of Borrelia burgdorferi strain 297 by mammalian host-specific signals. Infect. Immun. 69:3618-3627.

26. Hirano, T., T. Minamino, K. Namba, and R. M. Macnab. 2003. Substrate specificity classes and the recognition signal for Salmonella type III flagellar export. J. Bacteriol. 185:2485-2492.

27. Hodzic, E., S. Feng, K. J. Freet, D. L. Borjesson, and S. W. Barthold. 2002. Borrelia burgdorferi population kinetics and selected gene expression at the host-vector interface. Infect. Immun. 70:3382-8.

28. Hudson, C. R., J. G. Frye, F. D. Quinn, and F. C. Gherardini. 2001. Increased expression of Borrelia burgdorferi vlsE in response to human endothelial cell membranes. Mol. Microbiol. 41:229-39.

29. Karlinsey, J. E., J. Lonner, K. L. Brown, and K. T. Hughes. 2000. Translation/secretion coupling by type III secretion systems. Cell 102:48797.

30. Kimsey, R. B. and A. Spielman. 1990. Motility of Lyme disease spirochetes in fluids as viscous as the extracellular matrix. J. Infect. Dis. 162:1205-8.

31. Kinsella, N., P. Guerry, J. Cooney, and T. J. Trust. 1997. The flgE gene of Campylobacter coli is under the control of the alternative sigma factor sigma54. J. Bacteriol. 179:4647-4653.

32. Koopman, M. B., E. Baats, O. S. de Leeuw, B. A. van der Zeijst, and J. G. Kusters. 1993. Molecular analysis of a flagellar core protein gene of Serpulina (Treponema) hyodysenteriae. J. Gen. Microbiol. 139 (Pt 8):1701-1706. 
33. Kostrzynska, M., J. D. Betts, J. W. Austin, and T. J. Trust. 1991. Identification, characterization, and spatial localization of two flagellin species in Helicobacter pylori flagella. J. Bacteriol. 173:937-946.

34. Kutsukake, K. and T. lino. 1994. Role of the FliA-FlgM regulatory system on the transcriptional control of the flagellar regulon and flagellar formation in Salmonella typhimurium. J. Bacteriol. 176:3598-605.

35. Li, C., A. Motaleb, M. Sal, S. F. Goldstein, and N. W. Charon. 2000. Spirochete periplasmic flagella and motility. J. Mol. Microbiol. Biotechnol. 2:345-54.

36. Lux, R., J. N. Miller, N. H. Park, and W. Shi. 2001. Motility and chemotaxis in tissue penetration of oral epithelial cell layers by Treponema denticola. Infect. Immun. 69:6276-83.

37. Lux, R., A. Moter, and W. Shi. 2000. Chemotaxis in pathogenic spirochetes: directed movement toward targeting tissues? J. Mol. Microbiol. Biotechnol. 2:355-64.

38. Matz, C., A. H. van Vliet, J. M. Ketley, and C. W. Penn. 2002. Mutational and transcriptional analysis of the Campylobacter jejuni flagellar biosynthesis gene flhB. Microbiology 148:1679-1685.

39. Motaleb, M. A., L. Corum, J. L. Bono, A. F. Elias, P. Rosa, D. S. Samuels, and N. W. Charon. 2000. Borrelia burgdorferi periplasmic flagella have both skeletal and motility functions. Proc. Natl. Acad. Sci. USA 97:10899-904.

40. Motaleb, M. A., M. S. Sal, and N. W. Charon. 2004. The decrease in FlaA observed in a flaB mutant of Borrelia burgdorferi occurs posttranscriptionally. J. Bacteriol. 186:3703-3711.

41. O'Toole, P. W., M. Kostrzynska, and T. J. Trust. 1994. Non-motile mutants of Helicobacter pylori and Helicobacter mustelae defective in flagellar hook production. Mol. Microbiol. 14:691-703.

42. Prouty, M. G., N. E. Correa, and K. E. Klose. 2001. The novel sigma54and sigma28-dependent flagellar gene transcription hierarchy of Vibrio cholerae. Mol. Microbiol. 39:1595-609.

43. Rosey, E. L., M. J. Kennedy, and R. J. Yancey, Jr. 1996. Dual flaA1 flaB1 mutant of Serpulina hyodysenteriae expressing periplasmic flagella is severely attenuated in a murine model of swine dysentery. Infect. Immun. 64:4154-62. 
44. Ruby, J. D., H. Li, H. Kuramitsu, S. J. Norris, S. F. Goldstein, K. F. Buttle, and N. W. Charon. 1997. Relationship of Treponema denticola periplasmic flagella to irregular cell morphology. J. Bacteriol. 179:1628-35.

45. Sadziene, A., D. D. Thomas, V. G. Bundoc, S. C. Holt, and A. G. Barbour. 1991. A flagella-less mutant of Borrelia burgdorferi. Structural, molecular, and in vitro functional characterization. J. Clin. Invest 88:82-92.

46. Samuels, D. S. 1995. Electrotransformation of the Spirochete Borrelia burgdorferi, p. 253. In J. A. Nickoloff (ed.), Methods of Molecular Biology. Humana Press, Totowa NJ.

47. Sartakova, M. L., E. Y. Dobrikova, M. A. Motaleb, H. P. Godfrey, N. W. Charon, and F. C. Cabello. 2001. Complementation of a nonmotile flaB mutant of Borrelia burgdorferi by chromosomal Integration of a plasmid containing a wild-type flaB allele. J. Bacteriol. 183:6558-64.

48. Sellek, R. E., R. Escudero, H. Gil, I. Rodriguez, E. Chaparro, E. PerezPastrana, A. Vivo, and P. Anda. 2002. In Vitro Culture of Borrelia garinii Results in Loss of Flagella and Decreased Invasiveness. Infect. Immun. 70:4851-8.

49. Wilson, D. R. and T. J. Beveridge. 1993. Bacterial flagellar filaments and their component flagellins. Can. J. Microbiol. 39:451-472.

50. Wu, J. and A. Newton. 1997. Regulation of the Caulobacter flagellar gene hierarchy; not just for motility. Mol. Microbiol. 24:233-239. 
Chapter 4: The high molecular weight ladder of the periplasmic flagella hook in Borrelia burgdorferi.

Melanie Sal, Satoshi Shibata, Shin-Ichi Aizawa, and Nyles W. Charon

Unpublished data 


\section{Abstract}

Motility is an important factor in the virulence of Borrelia burgdorferi, the causative agent of Lyme disease. Motility is provided by the rotation of periplasmic flagella (PFs) located inside an outer membrane sheath in close proximity to the cell cylinder. Similar to the flagella from other bacteria, PFs are composed of the basal body, hook, and filament. The hook is a flexible tube that acts as a universal joint connecting the basal body to the filament. Due to the function of the hook, this structure must be strong enough to support the rotation of PF filaments. Using a new method for PF isolation, we were able to purify PFs with the hook-basal body structures intact. We further isolated the intact hookbasal body structures alone from these PFs by depolymerizing the filaments at low $\mathrm{pH}$. In characterizing the PF hook in $B$. burgdorferi, we find a ladder of multiple high molecular weight polypeptides that are reactive to hook antisera in wild type, but not in the flgE mutant cells SC-1. This high molecular weight ladder is present in cell lysate from strains B31 and N40, and in purified PFs from B31, confirming that the ladder is associated with the PF hook. Furthermore, purified hook-basal body structures from B31 show a strong high molecular weight band by Western blotting with affinity purified FlgE antisera. Similar to the PF hook in Treponema species, this ladder pattern suggests that the PF hook may be crosslinked in B. burgdorferi. The nature of possible hook cross-linking is unknown at this time. However, evidence suggests that disulfide bond formation is not solely responsible for the tight association of hook subunits. We also find that, although FlgE monomer is made in a fliF mutant, it appears to be degraded and the high molecular weight ladder is absent. We reason that hook polymerization cannot 
occur in the absence of basal body formation. Further research will be necessary to understand the nature of hook subunit association in B. burgdorferi. 


\section{Introduction}

Borrelia burgdorferi, the causative agent for Lyme disease, is a highly motile wave-like spirochete. This spirochete is transmitted between mammalian and arthropod hosts. B. burgdorferi migrates from the midgut of a tick to the site of inoculation in the mammalian host during a blood meal (25). After being deposited in the skin, the spirochetes disseminate throughout the body to cause disease (25). The highly invasive nature of spirochetes is thought to be due, in part, to their unique ability to traverse highly viscous gel-like media, like that found in the extracellular matrix $(6,9,10)$. As a result, motility is considered to be a virulence factor for $B$. burgdorferi.

Motility is provided by rotation of periplasmic flagella (PFs). The PFs are inserted near the cell ends and extend inward, wrapping around the cell cylinder, to overlap in the middle of the cell. An outer membrane sheath covers both the cell cylinder and PFs. In general, a PF closely resembles the flagellum of other bacteria and is composed of a basal body, hook, and filament. The basal body is embedded in the cell cylinder and includes the motor machinery for rotation of the flagellum. Unlike the basal structure of enteric bacteria, B. burgdorferi has a basal body composed of a single ring, the MS ring, and lacks genes encoding $\mathrm{P}$ and $L$ rings (4). The complex filament of $B$. burgdorferi is composed of two flagellins, FlaB and FlaA (20). In other spirochetes, FlaB proteins make up the PF filament core surrounded by a FlaA sheath $(2,12-16)$. Although FlaA is associated with FlaB, the location of FlaA in B. burgdorferi has not yet been determined (20). FlaB was shown to be essential for motility in B. burgdorferi, 
and its presence contributes to the wave-like morphology of the cell $(20,24)$. Based on its role in other bacterial flagella, it is assumed that the PF hook in $B$. burgdorferi acts as a flexible coupling between the basal body and filament. The PF hook is essential for motility in spirochetes as demonstrated in both Treponema denticola and $B$. burgdorferi $(3,3,17,18)($ M. Sal manuscript in preparation). In addition, the absence of hook in B. burgdorferi impacts cell morphology and specifically reduces the accumulation of PF filament proteins FlaB and FlaA (M. Sal manuscript in preparation).

The PF hook, encoded by the flgE gene, is a protein of $46 \mathrm{kDa}$ that polymerizes to form the hook structure (M. Sal manuscript in preparation). $B$. burgdorferi flgE shares homology with flagellar hooks from other bacteria, and is highly conserved in spirochetes. The B. burgdorferi hook gene shares $57 \%$ identity with the hook gene from Leptospira interrogans, $43 \%$ identity with Treponema denticola, and 40\% identity with Escherichia coli (www.tigr.org). Examination of the PF hooks in Treponema denticola and Treponema phagedenis revealed a ladder of multiple high molecular weight polypeptides reactive to FlgE antiserum $(3,18)$. The ladder pattern indicated that the PF hook is cross-linked in those spirochetes. In contrast, Jwang et al. (8) looked for and found no evidence of a FlgE ladder pattern of high molecular weight polypeptides in B. burgdorferi strain N40. During analysis of the flgE::kan mutant in $B$. burgdorferi, SC-1, we generated specific antiserum to FlgE. To our surprise, as reported here, we found a high molecular weight ladder pattern similar to that 
found in Treponema species when we examined the PF hook of $B$. burgdorferi strain B31 by Western blotting with FlgE antiserum. We then sought to characterize the PF hook in more detail. 


\section{Materials and Methods}

Bacterial strains and growth conditions. The B. burgdorferi sensu stricto

strain $\mathrm{B} 31 \mathrm{~A}$ and strain $\mathrm{N} 40$ were grown at $33^{\circ} \mathrm{C}$ in $\mathrm{BSK}-\mathrm{Il}$ liquid in $3 \%$ carbon dioxide (1) $(19,23)$. The non-motile flgE mutant (SC-1), flaB mutant (MC-1), and fliF mutant cells were grown in liquid BSKII with $300 \mu \mathrm{g} / \mathrm{ml}$ of kanamycin.

PF Isolation. Cells were centrifuged at $8000 \mathrm{Xg}$ for 20 minutes, washed in phosphate buffered saline (PBS, $\mathrm{pH} 7.4$ ), and centrifuged at $8000 \mathrm{Xg}$ for 10 minutes at room temperature. Cell pellets were resuspended in PBS with a final concentration of $1 \% \mathrm{C}-14$ detergent, and gently shaken at $37^{\circ} \mathrm{C}$ for 30 minutes. Samples were centrifuged at $17,000 \mathrm{Xg}$ for 20 minutes at $4^{\circ} \mathrm{C}$. The supernatant fluid (S1) containing the PFs was moved to another tube and polyethylene glycol (PEG, 6000 mesh) was added to a final concentration of $2 \%$ and kept on ice. The pellet was resuspended in sucrose solution ( $0.5 \mathrm{M}$ sucrose, $0.15 \mathrm{M}$ TRIS, pH 8). Lysozyme $(0.1 \mathrm{mg} / \mathrm{ml})$ and disodium ethylene diamine tetra-acetate (EDTA, 2 $\mathrm{mM}$ ) were added and stirred on ice for 30 minutes, then at room temperature for 20 minutes. Detergent C-14 was added to a final concentration of $1 \%$, and stirred at room temperature for 30 minutes. Samples were centrifuged at 17,000 $\mathrm{Xg}$ for 10 minutes at $4^{\circ} \mathrm{C}$. PEG (2\%) was added to the supernatant (S2) and incubated on ice for 30 minutes. The supernatant samples S1 and S2 were centrifuged at $27,000 \mathrm{Xg}$ for 20 minutes at $4^{\circ} \mathrm{C}$. The pellets were washed in 10 $\mathrm{ml}$ water and ultracentrifuged at $210,000 \mathrm{Xg}$ for 30 minutes at $4^{\circ} \mathrm{C}$. Finally, isolated PFs pellets were resuspended in $1 \mathrm{ml}$ water. 
Hook-basal body purification. Isolated PFs were diluted in glycine buffer (50 $\mathrm{mM}$ glycine, $\mathrm{pH} 2.5$ ) and shaken gently at room temperature for 30 minutes. The PFs were then centrifuged at $303,800 \mathrm{Xg}$ for 30 minutes at $4^{\circ} \mathrm{C}$. The pellet containing hook-basal body structures was resuspended in water.

Electrophoresis and Western blotting. Approximately $10 \mathrm{ml}$ of late logrithmic phase cells were centrifuged at $8000 \mathrm{Xg}$ at $23^{\circ} \mathrm{C}$ for 10 minutes, washed with water, and centrifuged at $13,000 \mathrm{Xg}$ at $23^{\circ} \mathrm{C}$ for 5 minutes. The cell pellets were resuspended in $50 \mu \mathrm{l}$ of water and $50 \mu \mathrm{l}$ of sodium dodecyl sulfate (SDS) reducing buffer, and boiled for 5 minutes. Total protein from cell lysate was quantified by spectrophotometer using the BIO-RAD protein assay. Equal amounts of total protein from each sample were loaded onto a polyacrylamide gel. SDS-polyacrylamide gel electrophoresis (SDS-PAGE) and Western blotting with the enhanced chemiluminescent detection system $(E C L)$ were performed as previously described (5).

Antibody preparation. Polyclonal antiserum was raised against recombinant FlgE from B. burgdorferi as described elsewhere (M. Sal manuscript in preparation). The crude antiserum was adsorbed with acetone powder from strain BL21 Star (DE3)pLysS that did not harbor a protein expression plasmid (7). The sample containing serum and acetone powder was spun down, and the unreacted antiserum collected. An affinity column containing immobilized recombinant $B$. burgdorferi FlgE protein on an agarose support was constructed 
using the AminoLink Plus Immobilization Kit (Pierce). Next, the antiserum was passed through the affinity column, and the column was washed with buffer to remove non-specific antibodies. The FlgE specific antibodies were then eluted from the column using ImmunoPure IgG Elution Buffer (Pierce).

Microscopy. Cells and purified PFs were viewed by dark field microscopy.

Purified PFs and purified hook-basal bodies were viewed by electron microscopy using a JEOL JEM 1220 microscope at an accelerating voltage of $80 \mathrm{kV}$. 


\section{Results}

Isolation of complete PF filament, hook, and basal body. PFs were isolated as described in Methods and Materials. Using a new method whereby shearing was kept to a minimum, complete PFs were isolated with filament, hook, and basal structures intact (Figure 1). Antiserum to FlgE was used to decorate the PF hook in B. burgdorferi (Figure 1a,b). Although the hook structures appear to aggregate and staining is slightly darker at the hook region, further immunoelectron microscopy is needed to confirm the hook decoration with FlgE antiserum. Using a glycine buffer at low $\mathrm{pH}$, the PF filament was depolymerized leaving purified hook-basal body structures (Figure 1c). 

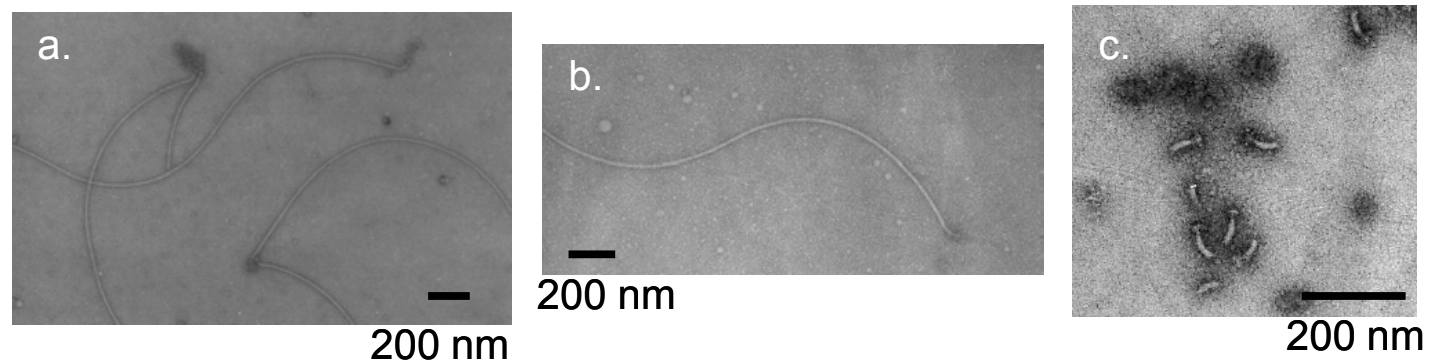

Figure 1. PF and hook-basal body isolation.

PFs purified from wild type B31 were incubated with crude polyclonal FlgE antiserum and viewed by electron microscopy at 10,000X magnification. Purified hook-basal body complexes viewed by electron microscopy at 20,000X magnification. 
FIgE detection by Western blotting. Using crude antiserum to identify the hook protein in wild type cell lysate by Western blotting, we found a ladder of high molecular weight bands reactive to the FlgE antibodies (Figure 3). To determine if these bands were related to the PF hook, we used purified PFs for Western blotting. The high molecular weight bands remained present during Western analysis of PFs (Figure 2). To further rule out non-specific antibody binding, the polyclonal antiserum was adsorbed by acetone powder from BL21 Star cells and purified by affinity column with recombinant FlgE protein. Western blotting with purified FlgE antibodies resulted in an identical pattern of high molecular weight proteins in PFs (Figure 2). The FlgE monomer was only present in PF samples that were boiled, suggesting that FlgE is stable in its polymerized form even in the presence of SDS and beta-mercaptoethanol in reducing buffer. Interestingly, the FlgE monomer and high molecular weight bands are not readily visible by Coomassie stained SDS-PAGE gel (Figure 2).

High molecular weight ladder of the PF hook. Western blot analysis of wild type cell lysates from B31 and N40 reveal the FlgE monomer of approximately 46 $\mathrm{kDa}$, which is absent in the flgE mutant SC-1. In addition, multiple high molecular weight polypeptides reactive to FlgE antisera are present in cell lysate from N40 and B31 wild type strains (Figure 3). Included in this ladder are bands at approximately $160 \mathrm{kDa}$, and others too high to estimate from molecular weight standards. These results are in contrast to those previously reported by Jwang, et al. (8) who did not find a high molecular weight FlgE ladder in strain N40. 
Crude polyclonal FlgE antiserum

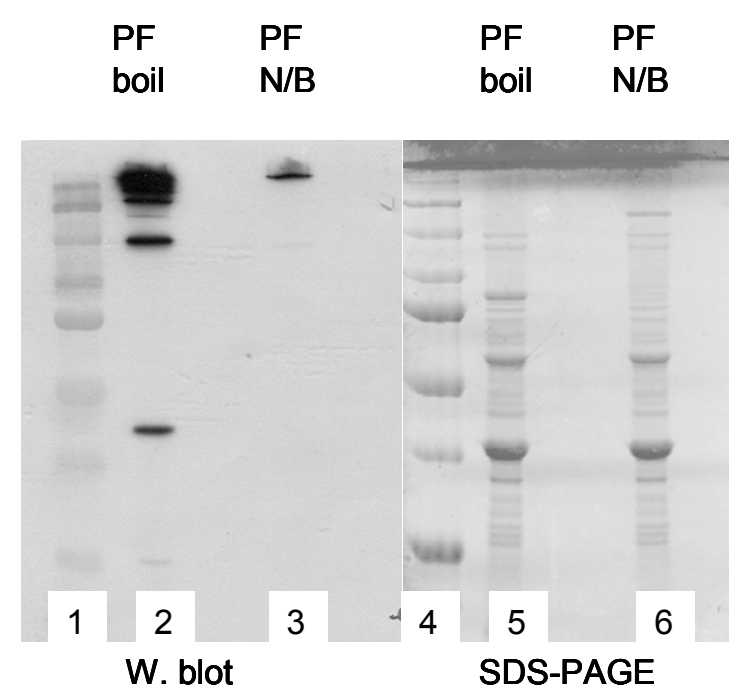

Affinity purified FlgE antibody

PF PF boil N/B

PF PF

boil N/B

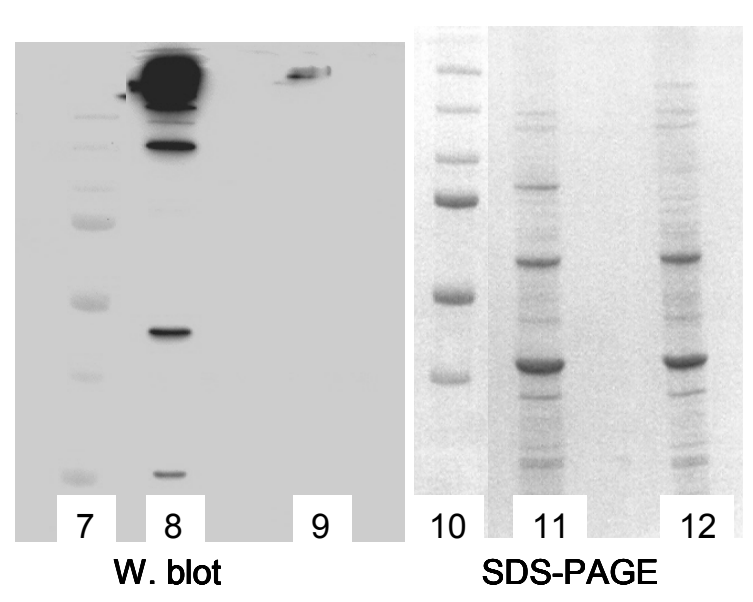

Figure 2. SDS-PAGE and Western blot of purified PFs with crude polyclonal FIgE antiserum and affinity purified FIgE antibodies.

For each SDS-PAGE and Western blot set, $10 \mu \mathrm{l}$ of purified PF sample was mixed thoroughly with $10 \mu \mathrm{l}$ of SDS reducing buffer. $10 \mu \mathrm{l}$ of the mixture was boiled for 5 minutes and allowed to cool. Each lane received $5 \mu$ of PF boiled sample (lanes 2 and 5) or PF not boiled sample (N/B: lanes 3 and 6). After the gel was run, it was cut such that one half was stained in Coomassie Brilliant blue and the other half was transferred to PDVF for Western blotting. Western blotting was performed using either crude polyclonal FlgE antiserum or affinity purified FlgE antibodies, followed by Protein A-HRP with ECL detection. Lanes $1,4,7$, and 10 are BioRad Dual Color molecular weight markers. 
To determine if these bands were related to PFs, we used purified PFs for Western blotting. The ladder of high molecular weight polypeptides were present in purified PFs lending further evidence that these polypeptides were aggregates of FlgE (Figure 3). Furthermore, a high molecular weight band was present in purified hook-basal body structures (Figure 3). The absence of FlgE monomer in purified hooks is likely an artifact of purification. After depolymerization of the filament, the sample is centrifuged to collect the pelleted intact hook-basal body structures. Any FlgE monomers present would likely be removed, as they are retained in the supernatant fluid with the flagellin monomers.

PF hook in motility mutants. Western blot analysis with crude polyclonal FlgE antiserum was used to investigate the assembly of polymerized hook structures in motility mutants. In Western blots using crude antiserum, there are two nonspecific bands that appear at $34 \mathrm{kDa}$ and $140 \mathrm{kDa}$ in all samples (compare Figures 3 and 4). These non-specific bands do not interfere with the FlgE reactive bands and are not present when blotting with affinity purified FlgE antibodies. The high molecular weight ladder found in wild type cells disappears along with the FlgE monomer in the flgE::kan mutant SC-1 (Figure 4). 


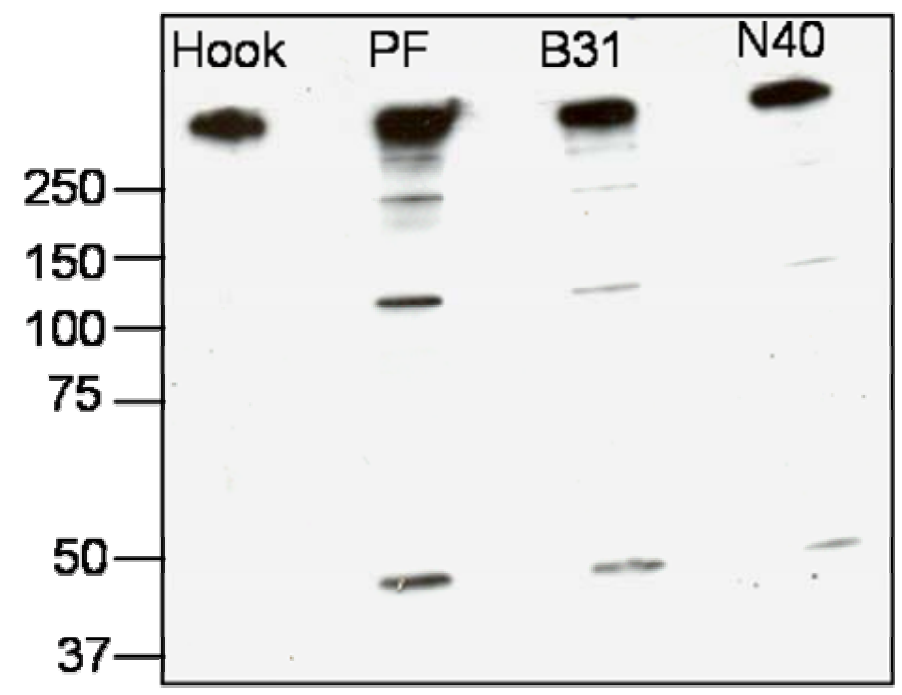

Figure 3. Western blot analysis of the PF hook.

Western blotting was carried out using affinity purified FlgE antibody with Protein A-HRP and ECL detection. Loaded in indicated lanes were $10 \mu \mathrm{g}$ of boiled cell lysate from strain B31 and N40 (lanes 3 and 4, respectively), $5 \mu$ of boiled PF sample from strain B31 (lane 2), and 10 $\mu$ l of boiled hook-basal body sample, isolated from the sample in lane 2 (lane 1). 
The high molecular weight ladder of FlgE in the flaB mutant MC-1 is the same as in wild type cells (Figure 4). These results suggest that the hook is fully formed in MC-1. However, in the fliF::kan basal body mutant, the high molecular weight ladder is absent (Figure 4). Furthermore, the FlgE monomer appears to be undergoing degradation in the fliF mutant cells. These results suggest that the fliF basal body mutant has produced FlgE, but the protein is unable to polymerize or form stable self-associations. 


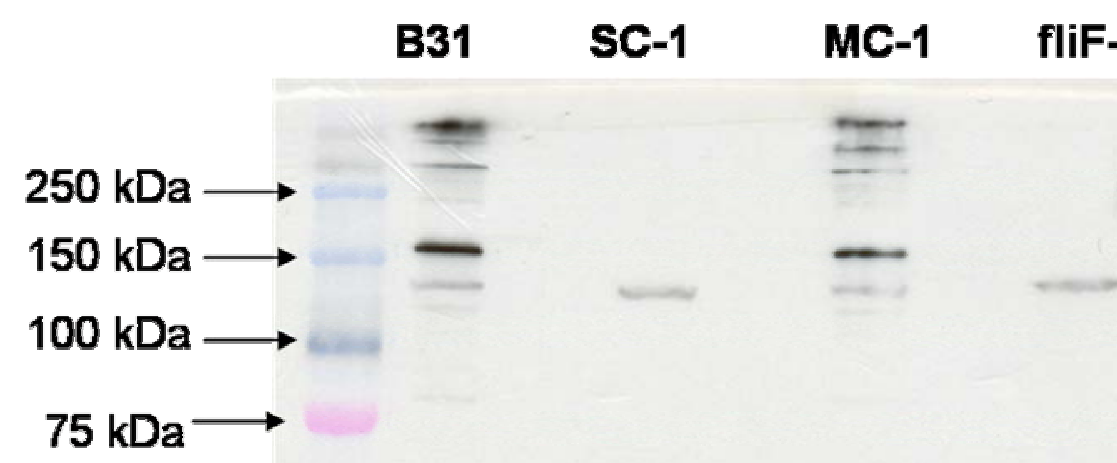

$50 \mathrm{kDa} \longrightarrow$

FlgE

$35 \mathrm{kDa} \longrightarrow$

Figure 4. Western blot of wild type and PF mutants with crude FlgE antiserum.

Each lane received $10 \mu \mathrm{g}$ of cell lysate protein from the indicated strain. Western blotting was performed using crude polyclonal FlgE antisera with Protein A-HRP and ECL detection. Two non-specific bands are present in all strains at approximately $34 \mathrm{kDa}$ and $140 \mathrm{kDa}$. 


\section{Discussion}

Microscopic and genetic evidence indicate that the periplasmic flagella of spirochetes are similar in structure and function to the external flagella of other bacteria. However, spirochete PFs are unique in regard to their location in the periplasmic space. PFs are constructed from many structural proteins and have complex filaments composed of multiple FlaB and FlaA flagellins. In the past, PFs were purified by means of shearing the flagella with glass beads. Although the shearing method provided ample PF filament with FlaA present, this harsh treatment resulted in the isolation of mostly PF fragments, rather than full length intact PFs. A new method for PF purification was developed such that hook and basal body structures remained intact on isolated PFs. This method was used to obtain purified PFs and further isolate hook-basal bodies for subsequent use in electron microscopy and Western blotting. With the new method for PF purification, one can determine the proximal and distal portions of a filament based on the location of the hook and basal structures. Having full length PFs with a point of reference will be invaluable for future immunoelectron microscopy experiments, such as localizing FlaA along the filament or localizing motor complexes on the basal body.

Relatively little is known about the hook and basal body structures in spirochetes. Previous studies indicate that the PF hooks of Trepomena denticola and Treponema phagedenis have a unique feature that is not described for flagellar hooks elsewhere. The hooks in these species demonstrate a ladder of high molecular weight bands during Western blotting with antibodies against the 
PF hook $(3,18)$. This ladder pattern suggests that the FlgE protein is crosslinked in PF hooks from these spirochetes. Our results indicate a similar high molecular weight ladder pattern of hook protein in B. burgdorferi.

We find that the high molecular weight ladder of polypeptides is present in whole cell lysate from strains B31 and N40. This finding contradicts the results previously reported by Jwang, et al. $\left(^{*}\right)$. It is possible that the high molecular weight signal was initially overlooked or considered to be non-specific antibody binding during Western blot. In our hands, the hook monomer and ladder are difficult to detect in SDS-PAGE gels because they do not produce strong signal with Coomassie brilliant blue (Figure 2) and silver staining (not shown).

When examining purified PFs, we find that the ladder and FlgE monomer are only detected when the PF samples are boiled. Coomassie stained gels illustrate that other flagella proteins, including the flagellins FlaB at $41 \mathrm{kDa}$ and FlaA at 38 $\mathrm{kDa}$, dissociate and migrate through the gel in samples that are not boiled. Furthermore, the isolated hook-basal body structures, verified by electron microscopy, retain a high molecular weight signal by Western blot even after sample boiling. These findings suggest a strong association of FlgE in the hook because it does not readily dissociate into its monomeric form. A similar phenomenon reported in PF hooks from treponemes is thought to due to subunit cross-linking, however the nature of the subunit association is unknown $(3,18)$. A common form of protein cross-linking is through the formation of disulfide 
bonds. However, the possible cross-linking of FlgE in B. burgdorferi PF hooks due to disulfide bond formation is unlikely for two reasons. First, the betamercaptoethanol present in SDS protein sample buffer should be sufficient to reduce disulfide linkages. Second, analysis of the amino acid sequence for FlgE reveals that there is a single cysteine near the center of the protein at residue 178 (8). Similarly, there is a single cysteine residue located near the center of the hook protein in T. phagedenis (18). In general, the center of the hook protein is not considered to be involved in protein binding; rather, the amino and carboxy-terminal regions of the FlgE protein are highly conserved and may act as the domains for subunit polymerization $(11,21,22,26)$. Moreover, the amino terminus of $B$. burgdorferi FlgE is $56 \%$ identical overall, and $85 \%$ identical at only the $\mathrm{N}$-terminus, of hook protein from $T$. phagedenis $(8,18)$. From the above information, it is reasonable to assume that the strength of the FlgE association cannot be attributed to disulfide bonds alone, but is likely to be similar in nature between $B$. burgdorferi and $T$. phagedenis.

The FlgE interaction that results in a hook ladder is only found in cells that have completed the construction of their basal bodies. We find complete high molecular weight ladder patterns in wild type and null mutants in flaB. In both of these cell types, construction of the flagellum should progress normally through the completion of the hook until filament formation. However, in the case of a fliF mutant, the basal body formation is incomplete. As a result, the hook cannot assemble. Our results are consistent with this idea as we find no high molecular 
weight bands reactive to FlgE antibodies to indicate aggregation of FlgE subunits in a fliF mutant. Furthermore, FlgE reactive bands appear below the monomer in the fliF mutant strain suggesting that the protein is undergoing degradation. Hook monomer degradation would be a likely event if the proteins were unable to assemble properly onto the flagellar structure, as demonstrated in the fliF mutant strain.

The data presented in this communication strongly suggest that the high molecular weight ladder pattern of polypeptides in $B$. burgdorferi is the PF hook. The high sequence identity of FlgE proteins in B. burgdorferi and T. denticola suggest that the PF hooks are similar in structure and function. Thus, the nature of the FlgE subunit association or cross-linking is likely similar as well. The unique ladder pattern described in these two spirochetes, but not for bacteria with external flagella, may result from the unusual demands placed on the PF. For instance, the PF hook may require cross-linking as structural reinforcement due to a lack of multiple rings used to anchor the basal body in the membrane. Alternatively, perhaps cross-linking is important to ensure that the hook remains curved and rigid to keep the PF filaments closely aligned with the spirochete cell body.

Further experimentation is required to elucidate the composition of the high molecular weight bands and the nature of FlgE crosslinking in $B$. burgdorferi. Sequence analysis is necessary to verify that the ladder is 
composed solely of FlgE. Complementation of flgE in SC-1 will be necessary to restore the expression of the hook monomer and ladder. If the ladder is proven to be FlgE, one may then be able to determine the nature of FlgE cross-linking in B. burgdorferi.

\section{Acknowledgements}

This research was funded by U.S. Public Health Service grant Al29743 to N.W.C, American Heart Association grant 0365225B to M.A.M., and the CREST Program organized by the Japan Science and Technology Agency. 
References

1. Bono, J. L., A. F. Elias, J. J. Kupko, B. Stevenson, K. Tilly, and P. Rosa. 2000. Efficient targeted mutagenesis in Borrelia burgdorferi. J. Bacteriol. 182:2445-52.

2. Charon, N. W., E. P. Greenberg, M. B. Koopman, and R. J. Limberger. 1992. Spirochete chemotaxis, motility, and the structure of the spirochetal periplasmic flagella. Res. Microbiol. 143:597-603.

3. Chi, B., R. J. Limberger, and H. K. Kuramitsu. 2002. Complementation of a Treponema denticola flgE mutant with a novel coumermycin A1resistant $T$. denticola shuttle vector system. Infect. Immun. 70:2233-2237.

4. Fraser, C. M., S. Casjens, W. M. Huang, G. G. Sutton, R. Clayton, R. Lathigra, O. White, K. A. Ketchum, R. Dodson, E. K. Hickey, M. Gwinn, B. Dougherty, J. F. Tomb, R. D. Fleischmann, D. Richardson, J. Peterson, A. R. Kerlavage, J. Quackenbush, S. Salzberg, M. Hanson, R. van Vugt, N. Palmer, M. D. Adams, J. Gocayne, J. C. Venter, and et al. 1997. Genomic sequence of a Lyme disease spirochaete, Borrelia burgdorferi. Nature 390:580-6.

5. Ge, Y., C. Li, L. Corum, C. A. Slaughter, and N. W. Charon. 1998. Structure and expression of the FlaA periplasmic flagellar protein of Borrelia burgdorferi. J. Bacteriol. 180:2418-25.

6. Greenberg, E. P. and E. Canale-Parola. 1977. Relationship between cell coiling and motility of spirochetes in viscous environments. J. Bacteriol. 131:960-9.

7. Harlow, E. and D. Lane. 1988. Antibodies: a laboratory manual, p. 63. Cold Spring Harbor, Cold Spring Harbor, NY.

8. Jwang, B., P. Dewing, E. Fikrig, and R. A. Flavell. 1995. The hook protein of Borrelia burgdorferi, encoded by the flgE gene, is serologically recognized in Lyme disease. Clin. Diagn. Lab Immunol. 2:609-615.

9. Kaiser, G. E. and R. N. Doetsch. 1975. Letter: Enhanced translational motion of Leptospira in viscous environments. Nature 255:656-657.

10. Kimsey, R. B. and A. Spielman. 1990. Motility of Lyme disease spirochetes in fluids as viscous as the extracellular matrix. J. Infect. Dis. 162:1205-8.

11. Kinsella, N., P. Guerry, J. Cooney, and T. J. Trust. 1997. The flgE gene of Campylobacter coli is under the control of the alternative sigma factor sigma54. J. Bacteriol. 179:4647-4653. 
12. Koopman, M. B., E. Baats, O. S. de Leeuw, B. A. van der Zeijst, and J. G. Kusters. 1993. Molecular analysis of a flagellar core protein gene of Serpulina (Treponema) hyodysenteriae. J. Gen. Microbiol. 139 ( Pt 8):1701-1706.

13. Koopman, M. B., E. Baats, C. J. van Vorstenbosch, B. A. van der Zeijst, and J. G. Kusters. 1992. The periplasmic flagella of Serpulina (Treponema) hyodysenteriae are composed of two sheath proteins and three core proteins. J. Gen. Microbiol. 138 ( Pt 12):2697-2706.

14. Koopman, M. B., O. S. de Leeuw, B. M. van der Zeijst, and J. G. Kusters. 1992. Cloning and DNA sequence analysis of a Serpulina (Treponema) hyodysenteriae gene encoding a periplasmic flagellar sheath protein. Infect. Immun. 60:2920-2925.

15. Li, C., L. Corum, D. Morgan, E. L. Rosey, T. B. Stanton, and N. W. Charon. 2000. The spirochete FlaA periplasmic flagellar sheath protein impacts flagellar helicity. J. Bacteriol. 182:6698-706.

16. Li, C., A. Motaleb, M. Sal, S. F. Goldstein, and N. W. Charon. 2000. Spirochete periplasmic flagella and motility. J. Mol. Microbiol. Biotechnol. 2:345-54.

17. Li, H., J. Ruby, N. Charon, and H. Kuramitsu. 1996. Gene inactivation in the oral spirochete Treponema denticola: construction of an flgE mutant. J. Bacteriol. 178:3664-3667.

18. Limberger, R. J., L. L. Slivienski, and W. A. Samsonoff. 1994. Genetic and biochemical analysis of the flagellar hook of Treponema phagedenis. J. Bacteriol. 176:3631-3637.

19. Motaleb, M. A., L. Corum, J. L. Bono, A. F. Elias, P. Rosa, D. S. Samuels, and N. W. Charon. 2000. Borrelia burgdorferi periplasmic flagella have both skeletal and motility functions. Proc. Natl. Acad. Sci. USA 97:10899-904.

20. Motaleb, M. A., M. S. Sal, and N. W. Charon. 2004. The decrease in FlaA observed in a flaB mutant of Borrelia burgdorferi occurs posttranscriptionally. J. Bacteriol. 186:3703-3711.

21. Mullin, D. A., N. Ohta, A. H. Mullin, and A. Newton. 2001. Organization, expression, and function of Caulobacter crescentus genes needed for assembly and function of the flagellar hook. Mol. Genet. Genomics 265:445-454.

22. Saito, T., T. Ueno, T. Kubori, S. Yamaguchi, T. lino, and S. I. Aizawa. 1998. Flagellar filament elongation can be impaired by mutations in the 
hook protein FlgE of Salmonella typhimurium: a possible role of the hook as a passage for the anti-sigma factor FlgM. Mol. Microbiol. 27:1129-1139.

23. Samuels, D. S. 1995. Electrotransformation of the Spirochete Borrelia burgdorferi, p. 253. In J. A. Nickoloff (ed.), Methods of Molecular Biology. Humana Press, Totowa NJ.

24. Sartakova, M. L., E. Y. Dobrikova, M. A. Motaleb, H. P. Godfrey, N. W. Charon, and F. C. Cabello. 2001. Complementation of a nonmotile flaB mutant of Borrelia burgdorferi by chromosomal Integration of a plasmid containing a wild-type flaB allele. J. Bacteriol. 183:6558-64.

25. Singh, S. K. and H. J. Girschick. 2004. Lyme borreliosis: from infection to autoimmunity. Clin. Microbiol. Infect. 10:598-614.

26. Vonderviszt, F., P. Zavodszky, M. Ishimura, H. Uedaira, and K. Namba. 1995. Structural organization and assembly of flagellar hook protein from Salmonella typhimurium. J. Mol. Biol. 251:520-532. 
Chapter 5. Production of Monoclonal Antibodies to the

Periplasmic Flagella Subunits of the Spirochete

Brachyspira hyodysenteriae

Melanie Sal, Chunhao Li, Linda Corum, Meenal Elliott, Nyles W. Charon 


\section{Abstract}

The periplasmic flagella (PFs) of Brachyspira hyodysenteriae, and most other spirochetes, have a complex composition and structure. Unlike bacteria with a single flagellin subunit, such as FliC in Escherichia coli, the PFs of $B$. hyodysenteriae have filament cores consisting of three immunologically crossreactive FlaB subunits (FlaB1, FlaB2, and FlaB3) and a sheath protein (FlaA). The FlaB proteins have a sequence identity of between 37 and $51 \%$, and are encoded by separate genes. The arrangement of FlaB subunits along the PF filament is unknown. As a first step in determining their arrangement, we hypothesized that we would be able to generate monoclonal antibodies (mABs) reactive to each FlaB protein. Intact PFs were purified from $B$. hyodysenteriae and used for immunization. After fusion of lymphocytes with a myeloma cell line, hybridomas were subcloned by limiting dilution and maintained in tissue culture. We isolated more than 40 positive clones reactive to different combinations of FlaB proteins. As observed by others, some mAbs were reactive to all three FlaB proteins. Although we were unable to generate mAbs specific to individual FlaB subunits, we isolated three clones that produced mAbs reactive to different combinations of two FlaB subunits. These mAbs, in conjunction with a panel of PF mutants, should facilitate determining the architecture of the PF filament by immunoelectron microscopy. Because other spirochetes, including Treponema pallidum, have a PF composition similar to $B$. hyodysenteriae, future findings may be applicable to the other species as well. 


\section{Introduction}

Spirochetes comprise a group of medically significant bacteria that are unique in both their morphology and motility. Spirochetes are known to cause such diseases as syphilis (Treponema pallidum), Lyme disease (Borrelia burgdorferi), and dysentery in swine (Brachyspira hyodysenteriae) (17). Spirochetes also contribute to periodontal disease (Treponema denticola)(17). The unusual morphological characteristics of spirochetes set them apart from other bacteria. Spirochetes are helical or wave-like in shape and utilize periplasmic flagella (PFs), rather than external flagella for locomotion. The unique features of their morphology and motility make the spirochetes quite adept at traversing viscous gel-like media, similar to the matrix found in host connective tissue $(1,6,8,10$, $11,21,24)$. Spirochete motility actually improves in viscous environments that impede the motility of other bacteria. Consequently, motility is considered a virulence factor for pathogenic spirochetes. The focus of research in our laboratory is to investigate the contribution of periplasmic flagella to the specialized motility of spirochetes and their pathogenesis.

B. hyodysenteriae has bundles of 7-9 PFs located at each cell pole that extend inward toward the center of the cell. As in most spirochetes, the PFs are composed of a FlaA protein sheath surrounding a FlaB protein core $(14,15)$. Each of the three $B$. hyodysenteriae PF filament core proteins is transcribed monocistronically, and the flaB1, flaB2, and flaB3 genes have been cloned and sequenced $(13-15,19,22)$. Inactivation of each FlaB protein results in cells with altered motility despite retaining the other FlaB proteins and the ability to 
assemble PFs $(19,22)$. In addition to altered motility, individual and dual flaA and flaB1 mutants are deficient in colonizing murine mucosa $(9,23)$. This evidence suggests that the individual FlaB proteins are not absolutely necessary for PF assembly, but have a functional role in motility. Interestingly, analysis of a dual flaB1flaB2 mutant revealed that these cells lost motility and were unable to assemble PFs (17). These data indicate that the presence of either FlaB1 or FlaB2 is required for assembly of a functional flagellar filament.

The composition of a PF core is known, but its architecture is not. Immunoelectron microscopy was used as a method for viewing the complex structure of flagella in bacteria containing multiple flagellins $(2,7,14)(5)$. In immunogold electron microscopy, monospecific primary antibodies reactive to one flagellin species bind specifically to a region recognized on a flagellum. Secondary antibodies attached to gold beads bind the monospecific primary antibodies. The gold beads, clearly visible by electron microscopy, indicate the location of the specific flagellin protein of interest. This method was used to determine the location of flagella filament sheath and core proteins from Spirochaeta aurantia, Brachyspira (Serpulina, Treponema) hyodysenteriae, and Campylobacter coli $(2,7,14)$. Similarly, immunodecoration was used to determine the locations of three flagellin proteins in the complex filament of Caulobacter crescentus (5). Although the overall morphology of the $C$. crescentus filament was uniform, its three flagellins were found to reside in specific regions along the filament based on results obtained by immunoelectron 
microscopic analysis (5). From the past success of this method, we propose that it will be possible to use immunoelectron microscopy to determine the arrangement of $\mathrm{FlaB} 1, \mathrm{FlaB} 2$, and $\mathrm{FlaB} 3$ along the PF filament core of $B$. hyodysenteriae.

To investigate the role of PFs in spirochete motility, our laboratory has constructed a panel of $B$. hyodysenteriae single and double mutants deficient in PF filament proteins (16). In this communication, we will describe a panel of different FlaB specific antisera produced from subcloned hybridomas. The specificity of our monoclonal antibodies includes reactivity to epitopes from FlaB1FlaB2, FlaB2FlaB3, and FlaB1FlaB2FlaB3. Antibodies raised to $B$. hyodysenteriae PFs are often cross-reactive to common epitopes expressed on the antigenically related FlaB proteins (14). Sequence analysis predicts that the three FlaB proteins share significant homology $(13,14)$. In addition, some antisera raised to FlaB proteins are cross-reactive to PFs from Treponema species, and vice versa, illustrating the high sequence homology of FlaB proteins across species $(14,18,20)$. This finding indicates a high degree of conservation with regard to PF proteins among spirochetes. Therefore, B. hyodysenteriae may be considered a model for obtaining information regarding the complex filament architecture of other spirochetes with multiple PF core proteins. Using multiple combinations of the mutants and antisera available, one may now attempt to complete the definitive experiments for determining PF filament architecture in $B$. hyodysenteriae. 


\section{Materials and Methods}

Bacterial Strains. B. hyodysenteriae strains were grown in brain-heart infusion broth $(\mathrm{BHI})$ supplemented with $10 \%$ bovine calf serum at $35^{\circ} \mathrm{C}$ in a Coy chamber with 3-4\% oxygen. The strains used that are deficient in FlaA production are as follows: A204 (flaA::kan); flaA::cat, flaB1::kan; flaA:: kan, flaB2::cat; flaA::kan, flaB3::cat $(16,17,22)$.

Monoclonal Antibody Production. Approximately $100 \mu \mathrm{g}$ of purified periplasmic flagella from B. hyodysenteriae strain A204 was used to immunize each of four Balb/c mice with Freund's Complete Adjuvant. Mice were subsequently boosted twice using $100-150 \mu \mathrm{g}$ of purified PFs and Freund's Incomplete Adjuvant and antibody titers were monitored. Mice with elevated antibody titers were exanguinated, and their spleens and draining lymph nodes removed. Lymphocytes were incubated with a fusion partner, the immortal myeloma cell line P3.X63.Ag8.653, and sustained in tissue culture. Cell supernatants from each well were screened by ELISA and Western blotting to identify positive hybridomas. The hybridoma cells from positive wells were subcloned by limiting dilution to attain single colonies producing FlaB specific antibodies. Positive colonies were expanded for large volume production of monoclonal antibodies. The antibodies harvested from hybridomas in tissue culture were purified and concentrated. 
ELISA and Western blotting. ELISA and Western blotting techniques were used to verify antibody specificity to the FlaB proteins. B. hyodysenteriae PFs were purified by standard methods (16). The purified PFs from wild type and mutant cells were separated by sodium dodecyl sulphate-polyacrylamide gel electrophoresis (SDS-PAGE) and transferred to PVDF membrane. Western blotting was carried out using the enhanced chemiluminescent $(E C L)$ system. For ELISA using 96 well plates, wells were coated with $100 \mu \mathrm{l}$ of a $2 \mu \mathrm{g} / \mathrm{ml}$ antigen solution using purified PFs from the following strains: wild type, flaA mutant strain (A203), flaA-flaB1 mutant strain (B209), flaA-flaB2 mutant strain, or flaA-flaB3 mutant strain in Borate saline solution (49 $\mathrm{g}$ Boric acid, $76.3 \mathrm{~g}$ sodium borate, $35.1 \mathrm{~g}$ sodium chloride, water to final volume $8 \mathrm{~L}$ ). Plates were incubated at $37^{\circ} \mathrm{C}$ for 3 hours or at $4^{\circ} \mathrm{C}$ overnight. Wells were then blocked at room temperature for 30 minutes with $250 \mu$ of blocking solution PBS/BSA ( $1 \%$ bovine serum albumen, $0.1 \% \mathrm{NaN} 3$ in phosphate buffered saline) and washed three times with PBS. Serum samples or supernatant fluid from hybridoma cell culture was added to the wells in serial diltuions and incubated at $37^{\circ} \mathrm{C}$ for 3 hours or at $4^{\circ} \mathrm{C}$ overnight. Plates were again washed with PBS. AP-conjugated anti-mouse secondary antibody was added to the wells and incubated at $37^{\circ} \mathrm{C}$ for 3 hours or at $4^{\circ} \mathrm{C}$ overnight, and the plates were washed with PBS as before. Wells were loaded with $80 \mu$ of phosphatase substrate solution in phosphatase substrate buffer (Sigma). To stop color development, $20 \mu \mathrm{l}$ of $5 \mathrm{~N} \mathrm{NaOH}$ was added to the wells. Reactivity was assessed by measuring the absorbance at $405 \mathrm{~nm}$ and $655 \mathrm{~nm}$ on a spectrophotometer. 
Immunoelectron microscopy. To visually determine the arrangement of FlaB1, FlaB2, and FlaB3 along the PF filament core of B. hyodysenteriae, immunoelectron microscopy was used to examine wild type and mutant cells and purified PFs. PFs examined were all from strains deficient in FlaA, as this sheath protein will likely hinder antibody association with the FlaB core. Whole cells were washed in PBS and incubated in $1 \%$ TritonX-100 at $37^{\circ} \mathrm{C}$ for 30 minutes to disrupt the outer membrane sheath. The cells were then centrifuged at $8000 \mathrm{Xg}$, and suspended in water. The monoclonal antibodies used recognize FlaB1 and FlaB2, or FlaB2 and FlaB3 by ELISA and Western blot. Immunoelectron microscopy of PFs was performed by standard procedures (5). Samples were mounted on carbon-coated copper grids and stained with $2 \%$ phosphotungstic acid ( $\mathrm{pH} 7$ ). The grids were examined using a JEOL JEM 1220 electron microscope at an accelerating voltage of $80 \mathrm{kV}$. 


\section{Results}

Screening for FlaB reactivity. Antibody specificity from harvested lymphocytes was measured by ELISA against PF preparations from wild type and PF mutants of $B$. hyodysenteriae. Wells that were positive by ELISA were tested by Western blotting to verify PF protein specificity (Figure 1). Some antibodies that reacted in ELISA did not react well in Western blotting. Other antibodies, indicated in Figure 1, reacted well in both assays.

Lymphocytes producing PF reactive antibodies were fused with the partner immortal myeloma cell line P3.X63.Ag8.653 (12). The resultant hybridomas were again screened by ELISA and Western blot for antibody specificity (Figure 2). Wells containing hybridomas whose antibodies reacted to FlaB proteins with the same specificity in both ELISA and Western blot were considered for later subcloning.

Due to the antigenic relatedness of the PF filament core proteins, it was not unusual that many of the mAbs reacted to all three FlaB proteins. In addition, many mAbs reacted to FlaB1 and FlaB2, further indicating that these two proteins are closely related and share epitopes not found on FlaB3. Although fewer in number, we also isolated hybridomas producing mAbs reactive to FlaB1 and $\mathrm{FlaB} 3$, and $\mathrm{mAbs}$ reactive to FlaB2 and FlaB3. Finally, hybridomas were identified that produced mAbs reactive to two proteins, $35 \mathrm{kDa}$ and $29 \mathrm{kDa}$, commonly found to co-purify with $B$. hyodysenteriae PFs. 


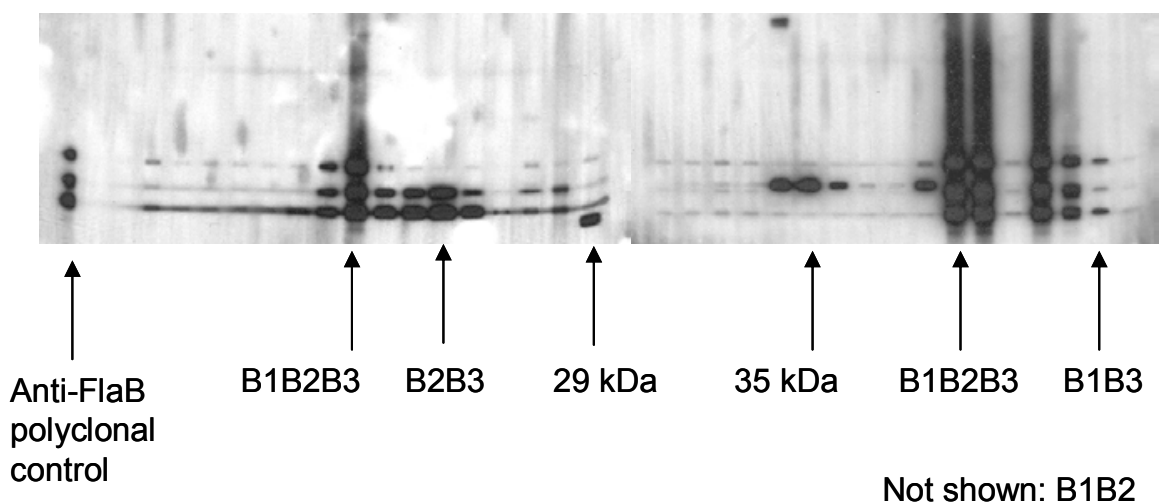

Figure 1. Lymphocytes producing antibodies reactive to FlaB proteins.

Western blotting was carried out on PF preparations from the flaA mutant strain A204 (22). Blots were incubated with culture supernatant fluid containing antibodies from harvested lymphocytes. Anti-mouse IgG conjugated to horseradish peroxidase was used as a secondary antibody with ECL detection. 
Hybridomas Expressing FlaB Reactive Monoclonal Antibodies

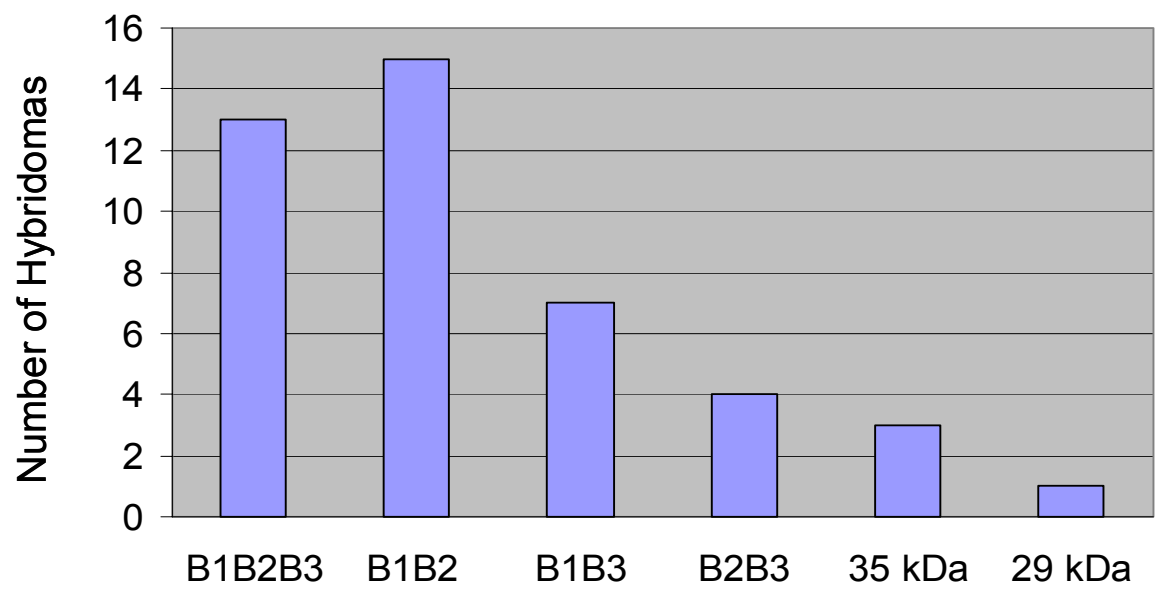

Monoclonal Antibody Specificity

Figure 2. Hybridomas identified and monoclonal antibody specificity.

Hybridomas were identified by microscopy and subcloned. Monoclonal antibodies produced were assayed by ELISA and Western blot. 
Unfortunately, no hybridomas were identified that produced antibodies reactive to a single FlaB by both ELISA and Western blot. From the hybridomas isolated, a few of particular interest were chosen for subcloning by limiting dilution (Figure 3). These included hybridoma 23C4, reactive to FlaB1 and FlaB2, and hybridoma 47B1, reactive to FlaB2 and FlaB3. One noteworthy antibody was strongly reactive to FlaB1 and FlaB2 by ELISA, but showed no reactivity by Western blotting (indicated with asterisks). As whole PFs were used to coat the ELISA plates, it is possible that these antibodies recognized an epitope present only when FlaB1 and FlaB2 are in association with one another. Although interesting, this particular hybridoma was not fully subcloned and characterized.

Culture supernatant media containing mAbs produced from hybridomas 23C4 and 47B1 were screened by Western blotting against a panel of PF mutants (Figure 4). The specificity of these monoclonal antibodies remained true across the entire panel of PF mutants. The results demonstrate that the loss of a particular FlaB protein does not negatively influence the antibody reactivity of the others. 


\section{$\underline{\text { Antibody }}$ Specificity}

$\begin{array}{cl}\text { 24A6 } & \text { FlaB1, FlaB2, FlaB3 } \\ \text { 22D2 } & \text { FlaB1, FlaB2, FlaB3 } \\ \text { 23C4 } & \text { FlaB1, FlaB2 } \\ \text { 47B1 } & \text { FlaB2, FlaB3 } \\ \text { 6C4 } & \text { 35KDa } \\ \text { 13D6 } & \text { *FlaB1 FlaB2* }\end{array}$

Figure 3. Hybridomas subcloned for monoclonal antibody production.

Hybridomas were subcloned by limiting dilution to yield colonies from single cells. Expansion of these cells in tissue culture yielded monoclonal antibodies of the indicated specificity. 


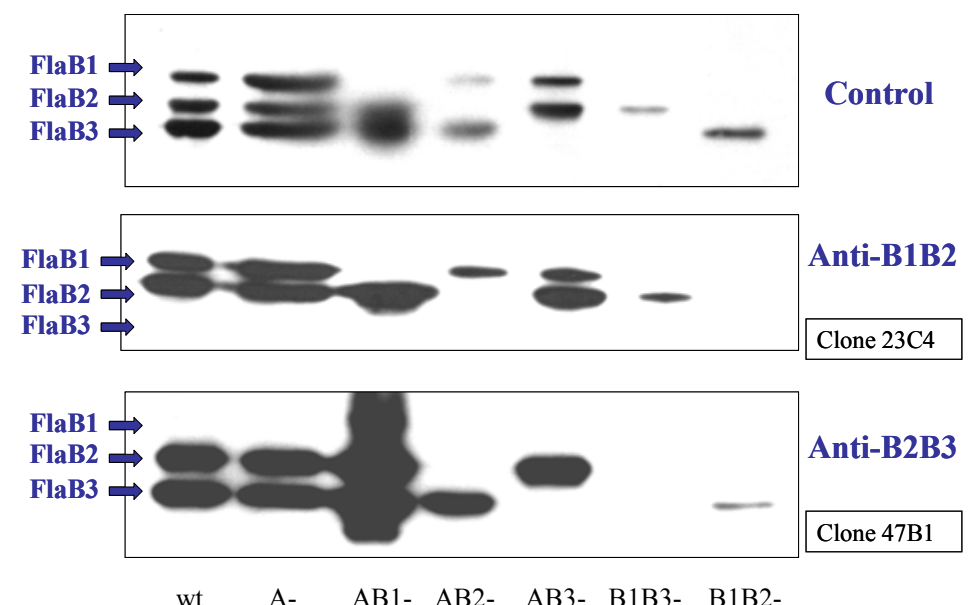

A- AB1- AB2- AB3- B1B3- B1B2-

Figure 4. Monoclonal antibody specificity across a panel of PF mutants.

Western blotting against a panel of PF mutants was carried out using culture supernatant fluid from hybridomas producing the monoclonal antibody indicated to the right. 
Location of FlaB Subunits in the PF Filament. The location of FlaB1, FlaB2, and FlaB3 on the PF filament is unknown. The mAbs produced could be used with PFs from wild type and mutant strains of $B$. hyodysenteriae to determine the distribution of FlaB proteins along the filament. Preliminary attempts at localizing FlaB proteins on the PF by electron microscopy failed due to high background (Figure 5). Two factors contributing to high background include sample purity and antibody concentration. The whole cell and PF samples contained a great deal of contaminating cellular matter, most likely outer membrane material that readily took up stain. Without highly purified cell and PF samples, antibody binding and visualization were difficult and results were inconclusive at best. Initially, whole cells were necessary for providing a point of reference to determine proximal and distal portions of a PF. However, a new method for isolating PFs with the hook and basal structures intact may improve the chances for success with immunolocalization experiments using only purified PFs. 


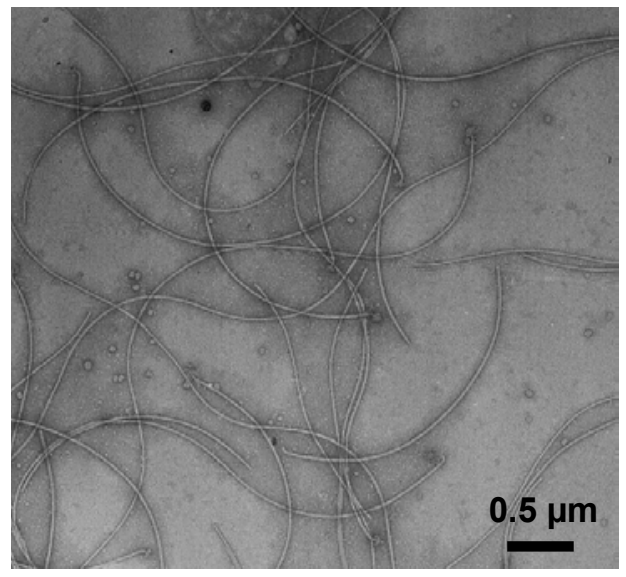

Figure 5. Electron micrographs of $B$. hyodysenteriae whole cells and PF.

PFs were purified using a new method (described in Chapter 4), and stained with 2\% PTA, pH 7. 
Models for the arrangement of FlaB proteins on a PF. Several models were considered to explain the orientation of FlaB subunit on the PF (Figure 6). Three basic models are described below. The first illustration depicts a PF with the FlaA sheath intact. Under those circumstances, antibody binding to the PF core would be minimal. Therefore, immunodecoration studies will be more productive using PFs deficient in the FlaA sheath protein. In the following models, the three FlaB proteins could be located in individual domains along the PF, shown in the first two PF illustrations. This arrangement would resemble the flagellar filament identified in $C$. crescentus $(3,5)$. The third illustration suggests a model that uses FlaB1 and FlaB2 together to make up a portion of the filament, while FlaB3 comprises a separate domain along the PF. This arrangement incorporates the domain motif, similar to C. crescentus, with a random assortment motif on a single PF filament. In the illustration, the product of flaB3, which is transcribed by a different promoter than the other two flaB genes, resides in its own domain while FlaB1 and FlaB2, which are thought to have overlapping function, randomly assemble to constitute another domain (3). In the last model, FlaB1, FlaB2, and FlaB3 are distributed throughout the filament in a mixed order or random arrangement (3). This arrangement might be analogous to the arrangement of the two flagellins in $C$. coli which are distributed along the length of the flagellum (7). In addition, the PF models illustrate possible results from immunodecoration with a monospecific antibody, such as FlaB2 for example. 


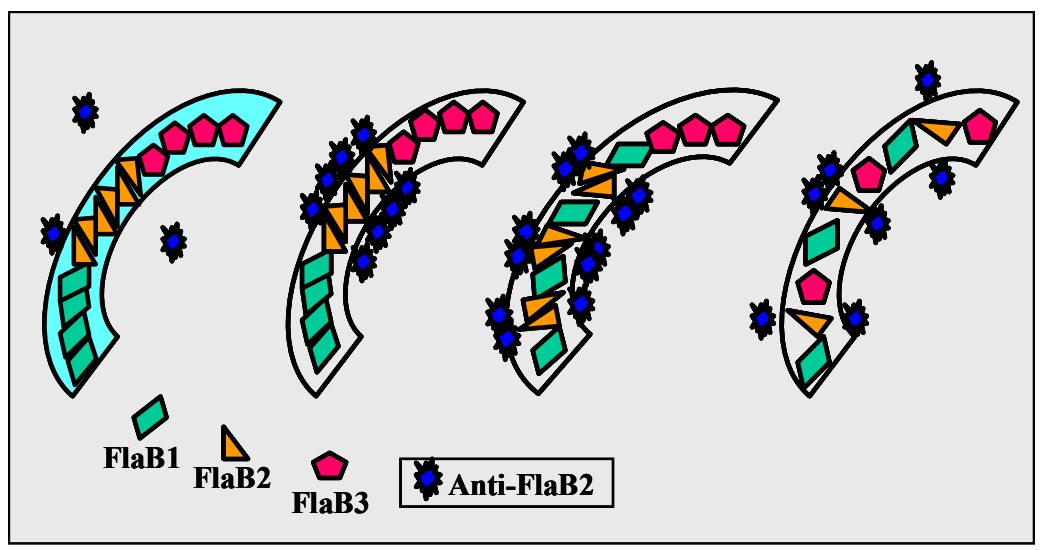

Figure 6. Model of possible FlaB arrangement on a PF filament. 


\section{Discussion}

The organelles for motility are located in the space between the cell cylinder and outer membrane sheath in spirochetes. Periplasmic flagella are similar to flagella from other bacteria in that they are composed of basal body, hook, and filament structures. Unlike the flagellar filaments of well described enteric bacteria, the spirochete PFs have complex filaments composed of multiple flagellins. Microscopy and SDS-PAGE analysis of purified spirochete PFs demonstrate that these structures include one or more FlaB proteins in the filament core and FlaA proteins forming a sheath around the filament core $(2,14$, 20, 25). Specifically, the PF filament of $B$. hyodysenteriae is composed of three different FlaB proteins and one FlaA protein $(13-15,19,22)$. Genetic analysis and characterization of targeted mutants demonstrated that each FlaB protein is encoded by a unique gene that is not located in close proximity to other flaB genes $(13,16,17,22)$. The reason for multiple FlaB proteins in spirochete PF filaments is unknown. Furthermore, the arrangement of FlaB proteins along the PF filament core is unknown. We proposed that it may be possible to determine the location of FlaB1, FlaB2, and FlaB3 along the PF filament core using immuno-electron microscopy with monoclonal antibodies specific to individual FlaB proteins. Therefore, our goal was to develop monoclonal antibodies with specific reactivity to individual FlaB proteins.

To acquire monoclonal antibodies against the FlaB proteins, we used a shotgun approach to isolate antibodies exhibiting a range of FlaB reactivity. Mice were immunized with preparations of whole PFs rather than individual FlaB 
proteins. In doing so, we anticipated that the mice would develop antibodies to epitopes present on intact PFs, as well as epitopes found in denatured FlaB proteins. Antibodies that recognize epitopes on intact PFs would be an asset for identifying FlaB subunits by immunoelectron microscopy. However, these same antibodies may not be as useful for other assays such as Western blotting. Therefore, we sought to characterize antibody specificity by both ELISA and Western blotting. Antibodies secreted by lymphocytes from immunized mice were characterized by ELISA using whole PFs to test for reactivity to epitopes on native FlaB proteins. In addition to ELISA, Western blotting was used to test for reactivity of antibodies to denatured FlaB epitopes. Lymphocytes producing FlaB reactive antibodies that performed well in both assays were immortalized by fusion with the myeloma cell line, and the resultant hybridomas were subcloned and further characterized.

The majority of hybridomas isolated produced antibodies reactive to FlaB1 and FlaB2 or reactive to all three FlaB proteins. These results were not surprising considering the antigenic relatedness of the three FlaB proteins (13). Antisera raised previously to individual FlaA and FlaB proteins from $B$. hyodysenteriae PFs cross-reacted with all FlaB proteins (14). Furthermore, antisera raised to other spirochete PFs can also cross-react with flagellins from B. hyodysenteriae (14). The amino acid sequences of the three FlaB proteins share identities of between 37 and $51 \%$, and are conserved among PFs from other spirochetes as well $(4,13,16)$. The greatest homology is found with FlaB1 
and FlaB2, which may explain why most antibodies cross-reacted with these two flagellins. In addition, evidence suggests that FlaB1 and FlaB2 may have overlapping function, as mutation in both flagellin genes renders a cell non-motile $(3,17)$. The closely related sequence and function of FlaB1 and FlaB2 suggests that these proteins will also share many similar epitopes making it difficult to isolate antibodies with specific reactivity to only one FlaB. As no monoclonal antibodies were identified with specific reactivity to a single FlaB protein exclusively, our results are consistent with the idea that the three FlaB flagellins are highly antigenically related.

The FlaB3 protein is less similar to either of the other flagellins than are FlaB1 to FlaB2. In addition, the FlaB3 protein is driven from a different type of promoter than FlaB1 and FlaB2. Finally, unlike the necessity for either FlaB1 or FlaB2, FlaB3 is not essential for synthesis of a PF to support motility (16). With the subtle differences in FlaB3, it is likely that this protein will display some unique epitopes which can be recognized by an antibody. Our results were consistent with FlaB3 having different epitopes than FlaB1 or FlaB2. The next most abundant types of monoclonal antibodies, after FlaB1 and FlaB2, were reactive to FlaB1 and FlaB3, or to FlaB2 and FlaB3. These results suggest that FlaB1 and FlaB3 share epitopes that are not present on FlaB2. Likewise, FlaB2 and FlaB3 share certain epitopes that are not present on FlaB1. 
The antibodies against FlaB1 and FlaB2, and those against FlaB2 and FlaB3, were characterized by ELISA and Western blotting against a panel of PF mutants. Our data show that the antibodies retain their reactivity to present flagellins even in the absence of other flagellins. These results suggest that the antibody to FlaB1 and FlaB2 may be able to recognize FlaB2 in a FlaB1 mutant, and vice versa. Using a combination of the two monoclonal antibodies with a combination of PF mutants, one may be able to determine the location of flagellins along a PF by immunoelectron microscopy. However, some problems exist with the execution of this experiment. First, although useful in ELISA and Western blotting, these monoclonal antibodies may not be adequate for use in immunoelectron microscopy. Secondly, the results obtained from mutant analysis with only two flagellins present may not give an accurate representation of the wild type PF filament architecture with three flagellins present. Finally, a point of reference will be required to determine which end of a PF is proximal versus distal to map the locations of FlaB1, FlaB2, and FlaB3 along the PF filament of $B$. hyodysenteriae.

Although immunoelectron microscopy has been attempted in the past to determine the flagellar filament architecture, immunodecoration with antisera raised against FlaB1 of $B$. hyodysenteriae cross-reacted with FlaB2 and FlaB3 to decorate the PF core, rather than the FlaB1 subunits only (14). In other bacteria, immunodecoration experiments were typically conducted with antibodies reactive to one flagellin protein exclusively $(2,5,7)$. In those experiments with $C$. 
crescentus, the arrangement of flagellins occurred in domains along the filament, similar to our first proposed model for B. hyodysenteriae (5). However, the spirochete PF filament may have an arrangement that differs from the complex flagellar filaments of $C$. crescentus, and the latter possible models may better represent the arrangement of FlaB proteins. Presently, the tools available for this type of protein identification in $B$. hyodysenteriae make the immunoelectron microscopy experiment quite difficult. Very recent advances in PF preparation techniques now allow for the identification of proximal and distal ends of a filament based on the presence of intact hook and basal structures on an isolated PF (See Chapter 4). With further experimentation, determination of the arrangement of FlaB proteins along the PF filament of $B$. hyodysenteriae may be possible by immunoelectron microscopy.

\section{Acknowledgements.}

I would like to thank Diane Berry for assistance with electron microscopy. I also thank Satoshi Shibata and Shin-Ichi Aizawa for expertise in developing the new method for PF preparation and for expertise in electron microscopy.

This research was supported by Public Health Service grant DE12046 and by USDA grant 95-37204-2132. 


\section{References}

1. Berg, H. C. and L. Turner. 1979. Movement of microorganisms in viscous environments. Nature 278:349-51.

2. Brahamsha, B. and E. P. Greenberg. 1988. Biochemical and cytological analysis of the complex periplasmic flagella from Spirochaeta aurantia. J. Bacteriol. 170:4023-4032.

3. Charon, N. W. and S. F. Goldstein. 2002. Genetics of motility and chemotaxis of a facinating group of bacteria: The Spirochetes. Annu. Rev. Genet. 36:47-73.

4. Charon, N. W., E. P. Greenberg, M. B. Koopman, and R. J. Limberger. 1992. Spirochete chemotaxis, motility, and the structure of the spirochetal periplasmic flagella. Res. Microbiol. 143:597-603.

5. Driks, A., R. Bryan, L. Shapiro, and D. J. DeRosier. 1989. The organization of the Caulobacter crescentus flagellar filament. J. Mol. Biol. 206:627-636.

6. Greenberg, E. P. and E. Canale-Parola. 1977. Relationship between cell coiling and motility of spirochetes in viscous environments. J. Bacteriol. 131:960-9.

7. Guerry, P., R. A. Alm, M. E. Power, S. M. Logan, and T. J. Trust. 1991. Role of two flagellin genes in Campylobacter motility. J. Bacteriol. 173:4757-4764.

8. Kaiser, G. E. and R. N. Doetsch. 1975. Letter: Enhanced translational motion of Leptospira in viscous environments. Nature 255:656-657.

9. Kennedy, M. J., E. L. Rosey, and R. J. Yancey, Jr. 1997. Characterization of flaA- and flaB- mutants of Serpulina hyodysenteriae: both flagellin subunits, FlaA and FlaB, are necessary for full motility and intestinal colonization. FEMS Microbiol. Lett. 153:119-28.

10. Kimsey, R. B. and A. Spielman. 1990. Motility of Lyme disease spirochetes in fluids as viscous as the extracellular matrix. J. Infect. Dis. 162:1205-8.

11. Klitorinos, A., P. Noble, R. Siboo, and E. C. Chan. 1993. Viscositydependent locomotion of oral spirochetes. Oral Microbiol. Immunol. 8:242244. 
12. Kohler, G. and C. Milstein. 1975. Continuous cultures of fused cells secreting antibody of predefined specificity. Nature 256:495-497.

13. Koopman, M. B., E. Baats, O. S. de Leeuw, B. A. van der Zeijst, and J. G. Kusters. 1993. Molecular analysis of a flagellar core protein gene of Serpulina (Treponema) hyodysenteriae. J. Gen. Microbiol. 139 ( Pt 8):1701-1706.

14. Koopman, M. B., E. Baats, C. J. van Vorstenbosch, B. A. van der Zeijst, and J. G. Kusters. 1992. The periplasmic flagella of Serpulina (Treponema) hyodysenteriae are composed of two sheath proteins and three core proteins. J. Gen. Microbiol. 138 ( Pt 12):2697-2706.

15. Koopman, M. B., O. S. de Leeuw, B. M. van der Zeijst, and J. G. Kusters. 1992. Cloning and DNA sequence analysis of a Serpulina (Treponema) hyodysenteriae gene encoding a periplasmic flagellar sheath protein. Infect. Immun. 60:2920-2925.

16. Li, C., L. Corum, D. Morgan, E. L. Rosey, T. B. Stanton, and N. W. Charon. 2000. The spirochete FlaA periplasmic flagellar sheath protein impacts flagellar helicity. J. Bacteriol. 182:6698-706.

17. Li, C., A. Motaleb, M. Sal, S. F. Goldstein, and N. W. Charon. 2000. Spirochete periplasmic flagella and motility. J. Mol. Microbiol. Biotechnol. 2:345-54.

18. Limberger, R. J. and N. W. Charon. 1986. Treponema phagedenis has at least two proteins residing together on its periplasmic flagella. J. Bacteriol. 166:105-12.

19. Motaleb, M. A., L. Corum, J. L. Bono, A. F. Elias, P. Rosa, D. S. Samuels, and N. W. Charon. 2000. Borrelia burgdorferi periplasmic flagella have both skeletal and motility functions. Proc. Natl. Acad. Sci. USA 97:10899-904.

20. Norris, S. J., N. W. Charon, R. G. Cook, M. D. Fuentes, and R. J. Limberger. 1988. Antigenic relatedness and $\mathrm{N}$-terminal sequence homology define two classes of periplasmic flagellar proteins of Treponema pallidum subsp. pallidum and Treponema phagedenis. J. Bacteriol. 170:4072-4082.

21. Prpic, J. K., F. Trewartha, and S. R. Graves. 1981. Enhanced retention of motility and virulence of Treponema pallidum (Nichols strain) in vitro by the addition of gelatin to anaerobic medium. Sex. Transm. Dis. 8:1-4.

22. Rosey, E. L., M. J. Kennedy, D. K. Petrella, R. G. Ulrich, and R. J. Yancey, Jr. 1995. Inactivation of Serpulina hyodysenteriae flaA1 and 
flaB1 periplasmic flagellar genes by electroporation-mediated allelic exchange. J. Bacteriol. 177:5959-5970.

23. Rosey, E. L., M. J. Kennedy, and R. J. Yancey, Jr. 1996. Dual flaA1 flaB1 mutant of Serpulina hyodysenteriae expressing periplasmic flagella is severely attenuated in a murine model of swine dysentery. Infect. Immun. 64:4154-62.

24. Ruby, J. D. and N. W. Charon. 1998. Effect of temperature and viscosity on the motility of the spirochete Treponema denticola. FEMS Microbiol. Lett. 169:251-4.

25. Trueba, G. A., C. A. Bolin, and R. L. Zuerner. 1992. Characterization of the periplasmic flagellum proteins of Leptospira interrogans. J. Bacteriol. 174:4761-8. 


\section{Chapter 6: General Discussion}

In this section, I will discuss the importance of my work with regard to the advancement of research in the field of spirochete motility. My specific contribution to selected aims will be discussed in the context of achieving the overall goals of the project.

The decrease in FlaA observed in a flaB mutant of $B$. burgdorferi occurs post-transcriptionally.

One of the goals in this project was to estimate the relative amounts of FlaA and FlaB in wild type B. burgdorferi. In other spirochetes, FlaA forms a sheath around a FlaB filament core and is estimated to be of equal abundance to the FlaB proteins (6). However, FlaA in B. burgdorferi is considered a minor filament protein based on its apparent lesser abundance than FlaB by SDSPAGE (12). Since a loss of FlaA might occur during PF isolation, whole cell lysates were immunoblotted to determine the amount of FlaA in wild type cells. To obtain standards for quantitative immunoblotting, purified FlaA and FlaB were required. The PF preparation method used in our lab did not purify both FlaA and $\mathrm{FlaB}$, so further isolation was required to separate these two flagellins (8). Immunoprecipitation assays demonstrated that FlaA was associated with FlaB (12). Since these two flagellins coprecipitated, immunoprecipitation was not a viable means by which to purify FlaA from FlaB. 
My contribution to this project was to isolate pure FlaA and pure FlaB proteins to use as standards for quantitative immunoblotting. To accomplish this, I first purified PFs by standard methods in our laboratory and separated the flagellins by SDS-PAGE on large gels. After gel staining, individual protein bands were carefully cut from the gels, and electroelution was performed on the gel pieces to obtain purified proteins. After dialysis and concentration, the purified proteins were used to generate standard curves for quantitative immunoblotting to estimate the amount of FlaA and FlaB in B. burgdorferi. We found that the amount of $\mathrm{FlaB}, 10 \%$ of total cell protein, was in agreement with estimates of the relative amount of flagellin in other multi-flagellate bacteria (13). In addition, we estimated that FlaA constituted less than $0.5 \%$ of the total cellular protein. These data confirm that B. burgdorferi makes less FlaA than FlaB, which is an unusual finding for spirochete PFs. In Brachyspira and Treponema species, FlaA is found in approximately equal abundance to the multiple FlaB proteins (1). In these spirochetes, FlaA forms a sheath around the PF filament core (6). The finding that B. burgdorferi has considerably less FlaA than FlaB is not consistent with FlaA forming a sheath around the PF filament. The location and function of FlaA in B. burgdorferi is still under investigation.

An interesting observation in the flaB mutant MC-1 of $B$. burgdorferi was the marked reduction of FlaA protein accumulation. In other spirochetes, the inactivation of one flagellin gene does not dramatically alter the expression of other flagellins (14). For example, FlaA, FlaB2, and FlaB3 protein levels are not 
reduced when flaB1 is inactivated in $B$. hyodysenteriae (14). Because little is known about the regulation of flagella synthesis in B. burgdorferi, we sought to determine the mechanism behind the reduction of FlaA accumulation in MC-1. A common theme in other bacteria is the transcriptional cascade control of gene expression for regulating flagella synthesis. Therefore, we used quantitative RTPCR to determine if the reduction in FlaA was the result of regulation at the transcriptional level.

My contribution to this project was to develop and optimize a protocol for a two-step real-time quantitative RT-PCR to measure the amount of flaA message in MC-1. In addition to negative controls, there are three samples to include for quantification when performing real-time PCR: standards, unknown samples, and internal controls. The standard template used to generate a curve for sample quantification was wild type flaA cDNA. Unknown samples included cDNA obtained after reverse transcription of wild type or MC-1 total RNA using random hexamers as primers. Priming with random hexamers promotes the reverse transcription of all messenger RNA with the same efficiency. The benefit from this is two-fold. First, this allows us to use the same lot of cDNA to amplify our target $(f l a A)$ and our housekeeping gene, or internal control. Second, we eliminate any differences in quantification resulting from variable priming efficiencies of specific gene primers during reverse transcription when using two different lots of cDNA. The third sample to include during real-time PCR is the internal control, or housekeeping gene. The housekeeping gene is chosen on 
the basis that it is unrelated to the target gene of interest and therefore should not vary in its expression under the specific experimental conditions. One such gene used almost exclusively for PCR applications in B. burgdorferi is the flagellin gene flaB. A problem is inherent in using flaB as an internal control for gene expression analysis in the flaB mutant MC-1. Therefore, other options including enolase (eno) and 16S ribosomal RNA were explored. The results from our qRT-PCR experiments revealed that the 16S rRNA message used as an internal control remained at wild type levels in MC-1. Likewise, flaA was transcribed at wild type levels in MC-1. Therefore, in contrast to the regulation of flagella synthesis in other bacteria, flaA in $B$. burgdorferi is not regulated at the transcriptional level. Further analysis of FlaA indicated that protein turnover is involved in the regulation of this flagellin. The two-step real-time quantitative RTPCR methodology that I have described is now commonly used in our laboratory. It led to critical data for determining the message levels of fla $A$ and flaB in the flgE mutant SC-1, described previously in Chaper 3. Furthermore, it is in use as a tool for our laboratory to verify results obtained from DNA microarray analysis.

\section{Targeted Mutagenesis of the Periplasmic Flagellar Hook Gene in the Lyme Disease Spirochete Borrelia burgdorferi}


Although significant advances have been made in elucidating the structural role of PFs and the mechanism by which $B$. burgdorferi swims, little is known about the regulation of PF synthesis. Several lines of genetic evidence indicate that the regulation of PF synthesis is different than flagella synthesis in other bacteria. The most obvious feature is that $B$. burgdorferi does not utilize flagella specific transcriptional activators to regulate its PF synthesis in the same manner as other bacteria. Promoter sequences for motility genes in $B$. burgdorferi more closely resemble sigma-70 like consensus sequences than the flagella specific sigma-28 or sigma-54 sequences seen in other bacteria (9). Second, homologs for genes encoding sigma-28 and FlgM, the anti-sigma factor, have not been identified in $B$. burgdorferi (3). Clearly, B. burgdorferi is unique from other bacteria with regard to regulation of PF synthesis.

We sought to determine the role of the PF hook in this spirochete by inactivating the $f l g E$ gene and identifying what effects this would have on PF synthesis. As anticipated, the flgE mutant SC-1 had rod-shaped cell morphology, lacked PFs, and was non-motile. These results were in agreement with the previously described targeted flaB mutant, MC-1, that lacked PFs (11). Southern and PCR analyses revealed that the kan cassette was incorporated into the genome only once, at the predicted location within flgE, indicating that the aberrant insertion of a kan into a gene other than flgE was not responsible for the phenotype observed in SC-1. Products from genes downstream of flgE were expressed at wild type levels indicating that polar effects on downstream genes 
were not responsible for the phenotype of SC-1. The only PF proteins found to be affected in SC-1 were FlaA, FlaB, and FlgE.

In addition to its structural role, the flagellar hook plays an important regulatory role as a checkpoint during flagella synthesis in many bacteria. Although the structural aspect of the PF hook in B. burgdorferi was apparent, its regulatory role in PF synthesis was unknown. It is common for flagellin expression or accumulation to be inhibited under certain circumstances in other bacteria by transcriptional or translational regulation. Although both flagellin proteins were present at severely reduced levels in SC-1, transcriptional control of the flagellin genes in $B$. burgdorferi was unlikely because all motility genes have sigma-70 promoters. Quantitative RT-PCR, as described above, was used to determine the message levels of $f l a A$ and $f l a B$ in SC-1. I found that both flagellin genes were transcribed at wild type levels in SC-1. These data confirm that transcriptional regulation of fla $A$ and flaB cannot account for the reduction of flagellin protein in SC-1. Similar results were found for flaA message in MC-1.

It is evident that post-transcriptional regulation is responsible for the reduction of flagellin proteins in SC-1. Two possibilities for the posttranscriptional regulation include translational control and protein degradation. Spectinomycin was used to arrest translation in SC-1 and wild type cells, and flagellin protein levels were monitored over time to determine if protein turnover 
occurred in SC-1. As observed in MC-1, FlaA was found to decrease over time in SC-1 indicating that protein degradation is occurring. Interestingly, contrasting results were found for FlaB. SC-1 has markedly reduced levels of FlaB that remain stable over time in the presence of spectinomycin. These results suggest that protein turnover is not an appreciable cause of reduced FlaB protein levels in SC-1. Rather, translational control may be influencing FlaB accumulation in SC1.

The mutants examined thus far have demonstrated that PF synthesis is regulated at the post-transcriptional level by more than one mechanism. In both MC-1 and SC-1, FlaA protein is turned over which may account for the greatly reduced levels. However, FlaB is stable in SC-1 and is likely regulated at the translational level. We have determined from MC-1 that FlaB affects FlaA accumulation. We have determined from SC-1 that FlgE affects FlaB accumulation, which affects FlaA accumulation. Furthermore, I have consistently found that the amount of FlaA in SC-1 is less than that in MC-1. Perhaps there is another level of regulation, in addition to protein turnover, that affects FlaA accumulation in SC-1. From these studies, we are just beginning to understand the regulation of motility genes in B. burgdorferi. As with other bacteria, sorting out the process of PF regulation in B. burgdorferi will depend on designing and characterizing various motility mutants deficient in PF components. 


\section{High molecular weight ladder of FlgE in B. burgdorferi.}

An interesting observation was made regarding a high molecular weight ladder of polypeptides that were reactive to FlgE antiserum in Treponema denticola and Treponema phagedenis. These findings suggested that the PF hook is cross-linked in Treponema species $(2,10)$. Although it was reported previously that a high molecular weight ladder similar to that in Treponema was not present in B. burgdorferi strain N40, our Western blot results indicated otherwise (4). Whole cell lysates from wild type cells, strains N40 and B31, revealed a high molecular weight ladder of polypeptides that reacted strongly to FlgE polyclonal antiserum. The fact that this ladder pattern was not present in the flgE::kan mutant SC-1 lends support to its involvement with the PF hook. However, to ensure that this ladder did not result from non-specific binding, the antiserum was first adsorbed with acetone powder of BL21 Star (DE3) pLysS, then affinity purified with recombinant FlgE protein. Western blotting with affinity pure FlgE antibodies still revealed the ladder in wild type cells.

To further reduce the possibility that non-specific binding by the FlgE antibodies led to a ladder effect, we analyzed purified PFs to determine if the ladder was indeed composed of FlgE aggregates. Through collaboration with Dr. Shin-Ichi Aizawa's laboratory, we were able to optimize a new method for purifying PFs such that the hook and basal structures remain intact. This gentle 
method does not involve shearing flagella with glass beads, as a result we can isolate more fully intact PFs. The new PF isolation method now provides us with the ability to identify the proximal and distal ends of a PF filament, which may be helpful for future immunoelectron microscopy experiments. Furthermore, the new method has made it possible to purify hook-basal body complexes by depolymerizing the PF filament, while leaving the hook-basal body intact. We are now able to analyze just the hook-basal body complexes for the presence of FlgE ladder. Western blot analysis shows that the high molecular weight signal is present in purified hook-basal body complexes. Such evidence strongly suggests that the high molecular weight polypeptides recognized by FlgE antibodies are not artifacts from non-specific antibody binding, rather FlgE polymerizes to form aggregates that are likely cross-linked in B. burgdorferi.

As the FlgE-reactive ladder has been determined to be the PF hook, we checked cell lysates from PF mutants of $B$. burgdorferi. The ladder was present and identical to wild type cells in the PF filament mutant MC-1. These results were consistent with the notion that the PF hook must be fully intact before the addition of $\mathrm{FlaB}$, the protein whose gene was interrupted in MC-1. Unlike MC-1, the fliF::kan mutant did not have a ladder pattern, although FlgE monomer was present and appeared to be undergoing degradation. These interesting results suggest that FlgE cannot form strong self-associations and assemble into the PF hook if a constituent of the basal structure is absent. 
Evidence suggests that the PF hook in B. burgdorferi is cross-linked in a manner similar to that seen in $T$. phagedenis and $T$. denticola $(2,10)$. However, the nature of the crosslinking is unclear at this time. In $B$, burgdorferi, there is a single cysteine residue encoded in the amino acid sequence. Based on the sequence data and the maintenance of cross-linking in the presence of betamercaptoethanol from sample buffer, it seems unlikely that the cross-linking is based on disulfide bond formation. The cross-linking in T. phagedenis remained intact under various harsh conditions including boiling for 1 hour, $8 \mathrm{M}$ urea, $8 \mathrm{M}$ guanidine, $1 \mathrm{M}$ sodium hydroxide, beta-mercaptoethanol, and dithiothreitol (DTT) (10).

Future experiments are required to complete the characterization of the PF hook in B. burgdorferi. Complementation of SC-1, with the concomitant return of FlgE monomer and the ladder pattern, is critical for demonstrating that the ladder is indeed the PF hook structure. Our recent ability to isolate highly purified hook-basal body structures may allow us to determine the amino acid sequence of high molecular weight bands recovered from SDS-PAGE gels, which will unequivocally prove that the ladder pattern is composed of FlgE aggregates. 


\section{Monoclonal antibodies to the FlaB filament subunits of Brachyspira hyodysenteriae.}

Similar to many other spirochete species, Brachyspira hyodysenteriae has complex PF filaments composed of multiple flagellins. A common question regarding complex flagella filaments addresses the need for multiple flagellins to support motility. Why does $B$. byodysenteriae need so many flagellins when other bacteria, such as $E$. coli, function with just one? To tackle this question, one must first determine the role of each individual flagellin in a PF filament.

SDS-PAGE and Western blotting indicate that there are four prominent flagellins including FlaA, FlaB1, FlaB2, and FlaB3, and three other proteins of unknown function in purified PF preparations from B. hyodysenteriae (6). Based on microscopic analysis of spirochete PFs, evidence strongly suggests that FlaA forms a sheath around the PF filament core (7). The PF filament core consists of the three FlaB subunits. Each of the FlaB proteins is expressed from its own gene, and the three flaB genes are not located near one another in the genome in $B$. hyodysenteriae $(5,8,14,15)$. The three FlaB proteins are antigenically related and share approximately $37-51 \%$ amino acid identity (8). Previous results show that single mutants in any of the four flagellin genes are able to form PFs and support motility $(5,7,8,14)$. However, analysis of the non-motile flaB1FlaB2 double mutant indicates that FlaB1 and FlaB2 have overlapping 
function, as at least one of these proteins must be expressed to form a filament capable of supporting motility $(8,9)$. Interestingly, analysis of these genes reveals that flaB1 and flaB2 have flagella specific sigma-28 promoters, whereas flaB3 has a sigma-54 promoter. In addition to the implication of differential regulation of FlaB proteins, there are likely other differences between FlaB3 and the other two FlaB proteins with regard to the structure and function of PFs.

The location of FlaB1, FlaB2, and FlaB3 along the PF filament core is unknown in $B$. hyodysenteriae and other spirochete species. The first step in determining the architecture of the PF filament is to have the ability to identify individual FlaB proteins. Due to the antigenic relatedness of the flagellins, antiserum used for the identification of PF filament core in B. hyodysenteriae cross-reacts with all three FlaB proteins (6). To discriminate between the individual FlaB proteins, we attempted to isolate monoclonal antibodies (mAbs) with a specific reactivity to each FlaB protein. This task was difficult for a number of reasons. Cross-reaction of antiserum suggests that exposed regions of the filament recognized by antibodies are highly conserved between the three FlaB proteins. Therefore, it is necessary to isolate antibodies reactive to unique regions of the FlaB proteins, and these regions may be hidden. Antibodies to unique epitopes may be of little use if those regions are not surface exposed, as the specific antibodies are ultimately for use with intact PF structures visualized by immunoelectron microscopy. For this reason, intact PFs were used for 
immunization in hopes that a range of antibodies would be made that reacted to FlaB1, FlaB2, and FlaB3 separately or in combination.

After screening and subcloning a number of hybridomas, we found that there was still a high amount of cross-reactivity with monoclonal antibodies to the FlaB proteins. Monoclonal antibody reactivity to individual FlaB proteins was not achieved. However, two mAbs of interest were chosen due to their lack of reactivity with an individual FlaB. One mAb reacted with only FlaB1 and FlaB2. Another mAb reacted with only FlaB2 and FlaB3. These two mAbs were tested by ELISA and Western blotting against a panel of $B$. hyodysenteriae flagellin mutants indicating that they are reactive to both intact and denatured PFs. Unfortunately, our attempts at using these mAbs for immunoelectron microscopy have not yielded any conclusive results. Recent advances have been made in PF isolation methods that will allow immunodecoration of the intact PF alone, rather than using whole cells, to determine the arrangement of FlaB subunits on a PF filament. Further study using the mAb is necessary to determine the architecture of $B$. hyodysenteriae PF filaments by immunoelectron microscopy. 


\section{References}

1. Charon, N. W. and S. F. Goldstein. 2002. Genetics of motility and chemotaxis of a facinating group of bacteria: The Spirochetes. Annu. Rev. Genet. 36:47-73.

2. Chi, B., R. J. Limberger, and H. K. Kuramitsu. 2002. Complementation of a Treponema denticola flgE mutant with a novel coumermycin A1resistant T. denticola shuttle vector system. Infect. Immun. 70:2233-2237.

3. Fraser, C. M., S. Casjens, W. M. Huang, G. G. Sutton, R. Clayton, R. Lathigra, O. White, K. A. Ketchum, R. Dodson, E. K. Hickey, M. Gwinn, B. Dougherty, J. F. Tomb, R. D. Fleischmann, D. Richardson, J. Peterson, A. R. Kerlavage, J. Quackenbush, S. Salzberg, M. Hanson, R. van Vugt, N. Palmer, M. D. Adams, J. Gocayne, J. C. Venter, and et al. 1997. Genomic sequence of a Lyme disease spirochaete, Borrelia burgdorferi. Nature 390:580-6.

4. Jwang, B., P. Dewing, E. Fikrig, and R. A. Flavell. 1995. The hook protein of Borrelia burgdorferi, encoded by the flgE gene, is serologically recognized in Lyme disease. Clin. Diagn. Lab Immunol. 2:609-615.

5. Kennedy, M. J., E. L. Rosey, and R. J. Yancey, Jr. 1997. Characterization of flaA- and flaB- mutants of Serpulina hyodysenteriae: both flagellin subunits, FlaA and FlaB, are necessary for full motility and intestinal colonization. FEMS Microbiol. Lett. 153:119-28.

6. Koopman, M. B., E. Baats, C. J. van Vorstenbosch, B. A. van der Zeijst, and J. G. Kusters. 1992. The periplasmic flagella of Serpulina (Treponema) hyodysenteriae are composed of two sheath proteins and three core proteins. J. Gen. Microbiol. 138 ( Pt 12):2697-2706.

7. Koopman, M. B., O. S. de Leeuw, B. M. van der Zeijst, and J. G. Kusters. 1992. Cloning and DNA sequence analysis of a Serpulina (Treponema) hyodysenteriae gene encoding a periplasmic flagellar sheath protein. Infect. Immun. 60:2920-2925.

8. Li, C., L. Corum, D. Morgan, E. L. Rosey, T. B. Stanton, and N. W. Charon. 2000. The spirochete FlaA periplasmic flagellar sheath protein impacts flagellar helicity. J. Bacteriol. 182:6698-706.

9. Li, C., A. Motaleb, M. Sal, S. F. Goldstein, and N. W. Charon. 2000. Spirochete periplasmic flagella and motility. J. Mol. Microbiol. Biotechnol. 2:345-54. 
10. Limberger, R. J., L. L. Slivienski, and W. A. Samsonoff. 1994. Genetic and biochemical analysis of the flagellar hook of Treponema phagedenis. J. Bacteriol. 176:3631-3637.

11. Motaleb, M. A., L. Corum, J. L. Bono, A. F. Elias, P. Rosa, D. S. Samuels, and N. W. Charon. 2000. Borrelia burgdorferi periplasmic flagella have both skeletal and motility functions. Proc. Natl. Acad. Sci. USA 97:10899-904.

12. Motaleb, M. A., M. S. Sal, and N. W. Charon. 2004. The decrease in FlaA observed in a flaB mutant of Borrelia burgdorferi occurs posttranscriptionally. J. Bacteriol. 186:3703-3711.

13. Neidhardt, F. C. 1987. Escherichia coli and Salmonella typhimurium: cellular and molecular biology, p. 3-6. American Society for Microbiology, Washington D.C.

14. Rosey, E. L., M. J. Kennedy, D. K. Petrella, R. G. Ulrich, and R. J. Yancey, Jr. 1995. Inactivation of Serpulina hyodysenteriae flaA1 and flaB1 periplasmic flagellar genes by electroporation-mediated allelic exchange. J. Bacteriol. 177:5959-5970.

15. Zuerner, R. L. and T. B. Stanton. 1994. Physical and genetic map of the Serpulina hyodysenteriae B78T chromosome. J. Bacteriol. 176:10871092. 


\section{Appendix}

\section{Review articles:}

Li, C., Md. Abdul Motaleb, Melanie Sal, Stuart F. Goldstein, and Nyles W. Charon. (2000) Spirochete Periplasmic Flagella and Motility. J. Mol. Microbiol. Biotechnol. 2(4):345-354

Chunhao Li, Md. Abdul Motaleb, Melanie Sal, Stuart F. Goldstein, and Nyles W. Charon. (2001) Chapter 2: Gyrations, Rotations, Periplasmic Flagella: The Biology of Spirochete Motility. From: The Spirochetes: Molecular and Cellular Biology. Horizon Scientific Press, Wymondham, UK 
Curriculum Vitae 


\section{Melanie S. Sal}

\begin{tabular}{ll}
\hline \hline Department of Microbiology, & 514 Callen Avenue \\
Immunology, and Cell Biology & Morgantown, WV 26501 \\
West Virginia University & Phone (304)685-1577 \\
Morgantown, WV 26506-9177 & Email: msal@,hsc.wvu.edu
\end{tabular}

Phone: 304-293-2585

\section{Education}

West Virginia University, Morgantown, WV

Doctor of Philosophy, August 1998 - March 2005

Department of Microbiology, Immunology, and Cell Biology, School of Medicine

West Virginia University, Morgantown, WV

Bachelor of Arts, Biology, August 1992 - May 1997

West Virginia University, Morgantown, WV

Bachelor of Arts, Chemistry, August 1992 - May 1997

\section{Publications}

Sal M, Motaleb MA, Charon NW. (In preparation) Post-transcriptional flagellin regulation in a periplasmic flagellar hook mutant of Borrelia burgdorferi.

Motaleb MA, Sal MS, Charon NW. (2004) The decrease in FlaA observed in a flaB mutant of Borrelia burgdorferi occurs posttranscriptionally. J Bacteriol. 186(12):3703-11.

Li, C, MA Motaleb, M. Sal, SF Goldstein, and NW Charon. (2001) Gyrations, rotations, periplasmic flagella: The biology of spirochete motility. Chapter 2. In: The spirochetes: Molecular and Cellular Biology. Horizon Scientific Press, Wymondham, UK.

Li C, Motaleb A, Sal M, Goldstein SF, Charon NW. (2000) Spirochete periplasmic flagella and motility. J Mol Microbiol Biotechnol. 2(4):345-54. 


\section{Presentations}

104th General Meeting of the American Society for Microbiology, May 2004. New Orleans, Louisiana. "Monoclonal Antibodies to the Periplasmic Flagella Subunits of the Spirochete Brachyspira byodysenteriae." Melanie Sal (Poster presenter), Chunhao Li, Meenal Elliott, and Nyles W. Charon. West Virginia University.

Gordon Research Conference: Biology of Spirochetes. January 2004. Ventura, California. "The periplasmic flagellar protein FlaB influences FlaA expression in Borrelia burgdorferi" Md. A. Motaleb, Melanie S. Sal, and Nyles W. Charon. West Virginia University.

Bacterial Locomotion and Signal Transduction (BLAST) VII Meeting, January 2003. Cuernavaca, Mexico. "Targeted Mutagenesis of the Gene Encoding the Flagellar Hook Protein in the Lyme Disease Spirochete Borrelia burgdorferi." Melanie Sal (Poster presenter), Md. A. Motaleb, and N. W. Charon. West Virginia University.

Gordon Research Conference: Biology of Spirochetes. January 2002. Ventura, California. "Targeted Mutagenesis of the Gene Encoding the Flagellar Hook Protein in the Lyme Disease Spirochete Borrelia burgdorferi" Melanie Sal (Poster presenter), Md. A. Motaleb, and N. W. Charon. West Virginia University.

102 $^{\text {nd }}$ General Meeting of the American Society for Microbiology. May 2001. Orlando, Florida. "Targeted Mutagenesis of the Gene Encoding the Flagellar Hook Protein in the Lyme Disease Spirochete Borrelia burgdorferi" Melanie Sal (Poster presenter), Md. A. Motaleb, and N. W. Charon. West Virginia University.

\section{$>$ Teaching Experience}

Microbiology Laboratory, Teaching Assistant. 1999 Fall Semester.

Developed microbiology laboratory lectures. Assisted second year Medical Students with laboratory procedures including bacterial culture, staining, microscopy, and molecular biology techniques.

Microbiology 26/223 Laboratory, Teaching Assistant, 1999 Spring Semester.

Developed microbiology laboratory lectures and assisted undergraduate medical technology students. Prepared bacterial cultures, microscope slides, and student laboratory supplies.

Community Mini Medical School: Med School 101, Volunteer Instructor, October 2000. Demonstrated Gram staining and assisted parents of Medical Students in staining procedures and microscopy. Prepared bacterial strains, laboratory equipment and supplies. 
Adult Education Program at the Monongalia County Technical Education Center:

Volunteer, 2002-Present. Designed plasmids and prepared equipment for the "Crime Scene" genetics laboratory each semester.

Microbiology 200, Exam Proctor, 2002 - Present.

\section{Professional Societies}

American Society for Microbiology, 1999-Present.

\section{$>$ Honors and Awards}

Golden Key National Honour Society, 1996

Beta Beta Beta Biology Honorary, 1996

\section{Techniques}

Aerobic bacterial culture

Anaerobic bacterial culture

Molecular cloning techniques

Protein expression and purification

Real-time quantitative polymerase chain reaction

Western and Southern hybridization techniques

Monoclonal and polyclonal antibody production

Animal handling (mice, rabbits)

Tissue culture

Electron Microscopy 